\title{
EFFECT OF CYTOCHROME P-450 INDUCTION ON THE METABOLISM AND TOXICITY OF OCHRATOXIN A
}

\section{CENTRE FOR NEWFOUNDLAND STUDIES}

\section{TOTAL OF 10 PAGES ONLY MAY BE XEROXED}

(Without Author's Permission)

\section{RABEEA FAHMY OMAR}






\title{
Effect of Cytochrome P-450 Induction on The Metabolism and Toxicity of Ochratoxin A
}

\author{
by \\ C Rabeea Fahmy Omar, B.Sc.(Honours), M.Sc. \\ A thesis submitted to the School of Graduate \\ Studies in partial fulfilment of the \\ requirements for the degree of \\ Doctor of Philosophy
}

Biochemistry Department

Faculty of Science

Memorial University of Newfoundland

St. John's, Newfoundland

Canada A1B 3X9

October 1994 


\section{AiBSTRACT}

The role of cytochrome $\mathrm{P}-450$ in the stimulation of lipid peroxidation induced by the mycotoxin ochratoxin A (OTA) has been investigated. Purified cytochrome P-4.50 (1IB1) could effectively replace EDTA in stimulating lipid peroxidation in a reconstituted system consisting of phospholipid vesicles, NADPH-cytochrome P-450 reductase, Fe ${ }^{3+}$, EDTA and NADPII, suggesting that it could mediate the transfer of electrons from NADPH to Fe3t. Microsonws isolated from livers of cobalt protoporphyrin IX-treated rats (in which cytochrome P-450) was depleted; underwent OTA-dependent lipid peroxidation much more slowly than control microsomes.

The role of cytochrome P-450 in OTA metabolism was also investigated. To determine which cytochrome P-450 isoforms are involved in the metabolism of OTA, we used different cytochrome P-450 inducers to induce the major isoforms of cytochrome P-450) in the rat liver. Microsomes from these livers were used to investigate their effect on OTA metabolism. Pretreatment of rats with pregnenolone - 16o-carbonitrile (PCN), phenobarbital (PB), 3-methy] cholanthrene (3MC), and isosafrole (ISF) greatly induced 4(R)-4-OH-OTA formation; 4(S)-4-OI1OTA formation was also induced after pretreatment with $\mathrm{PB}, \mathrm{PCN}, 3 \mathrm{MC}$ and ISF. INI 1 pretreatment primarily induced the $4(S)$ isomer formation. The formation of the $4(R)$ and $4(S)$ isomers showed significant differences with respect to $\mathrm{pH}$ optima, effect of antioxidants and iron chelators. The $4(\mathrm{R})$ isomer formation showed a pH optimum of 6.0 using microsomes from rats treated with $3 \mathrm{MC}$ and ISF, and 6.5 using microsomes from rats treated with $\mathrm{PB}$ and $\mathrm{PCN}$ and was not inhibited by antioxidants or iron chelators. In contrast, both the $4(\mathrm{~S})$ isomer formation and lipid peroxidation showed a $\mathrm{pH}$ optimum of 7.0 - 7.5 and both activities were highly sensitive to inhibition by antioxidants and iron chelators. Lipid peroxides were not involved in the $4(\mathrm{~S})$ isomer formation since addition of linoleic acid hydroperoxide to microsomes did not give rise to the $4(\mathrm{~S})$ isomer. Cytochrome P-450 appeared to be essential since other hemoproteins such as horseradish 
peroxidase and hemoglobin were ineffective in metabolizing CTA. Microsomes from rats pretreated with Co-protoporphyrin IX resulted in no metabolism of ochratoxin A. 7-Ethoxy- and 7-pentoxyresorufin assays showed specificity towards cytochromes P-450 induced by $3 \mathrm{MC}$ (IA 1/IA2) and PB (IIB1) respectively. Also, metyrapone (inhibitor of cytochronie P-450 IIB1) preferentially inhibited OTA metzbolism by microsomes from rats treated with $\mathrm{PB}$, and \&-naphthoflavone (inhibitor of cytochrome P-450 LA1//A2) preferentially inhibited OTA metabolism by microsomes from $3 \mathrm{MC}$ and ISF treated rats. Monoclonal antibodies (MAbs) 1-7-1 (against P-450 IA1/IA2) and 2-66-3 (against P-450 JIB1) showed preferential inhibition of OTA metabolism by microsomes from $3 \mathrm{MC}$ and $\mathrm{PB}$ treated rats respectively.

Excretion of renal enzymes in urine is a sensitive non-invasive index of renal damage. Therefore, we examined the effect of cytochrome P-450 induction on the excretion of alkaline phosphatase and $\zeta$-glutamyl transferase ( $\gamma-\mathrm{CT})$ in a PB-treated rat group and a control rat group (both groups received O'TA). At the fourth day of OTA treatment higher enzyme levels were found in the control group compared to the $\mathrm{PB}$ group. The results provide evidence for the stimulatory role of " cytochrome P-450 in OTA-induced lipid peroxidation. Also, our data suggest that 4(R)-4OH-OTA is formed by normal mixed function oxidation but that 4(S)-4-OH-OTA formation may involve free iron. Also, our results show that the major cytochrome P-450 isoforms catalyzing OTA hydroxylation are IA1/LAZ (3MC and ISF), IIB1 (PB) and IIIAI/IIIA2 (PCN). The results from in vivo experiments suggest a protective effect of $\mathrm{PB}$ agairst $\mathrm{OTA}$ toxicity (through inducing cytochrome P-450, thus metnbolizing more OTA and excreting it faster from the body compared to controls). This was also evident from in vivo experiments of [ ${ }^{3 H] O T A}$ distribution. Therefore, OTA hydroxylation is more likely to be a detoxification pathway. 
iii

To my parents, my wife

and my daughter 


\section{ACKNOWLEDGEMENTS}

I would like to thank Dr. A.D. Rahimtula for his supervision and his valuable guidance throughout this work and also for giving me the opportunity to do research at MUN and for helping me to stand on my feet in the areas of toxicology and biochemistry. I also thank the members of my supervisory committee, Dr. E, Barnsley and Dr. Phil Davis for their constructive suggestions, guidance and support. A special thanks to Ms. Marie Codner for technical assistance and helping to make bench work a more enjoyable experience. I thank $\mathrm{my}$ friend Edward Randell for his valuable discussions and suggestions. Thanks also to Ms Gwyn Barrowman for her help in the OTA distribution experiment. Thanks to Dr. Joseph Banoub and to Nathalie Brunet for helping me running and interpreting NMR profiles of OTA. Thanks are extended to Towhid Bin Muzaffar for his help formating the thesis. I am also grateful to the members of Biochemistry Department for their kindness and support and for having me here. I am indebted to the School of Graduate Studies of Memorial University of Newfoundland for financial support in the form of fellowship. In particular, I would like to thank Dr. C. Sharpe for his kind support during the five and half years of my stay in St. John's. Thanks are also due to the Kidney Foundation of Canada for financially supporting this research.

I wish to extend special thanks to my parents for their kind blessings, and to my caring loving wife and daughter for their support and patience throughout my studies. 


\section{TABLE OF CONTENTS}

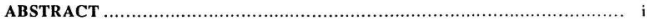

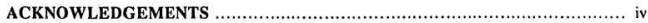

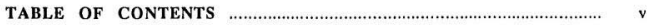

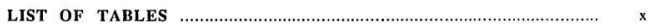

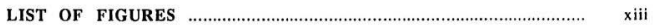

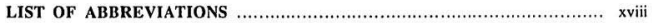

CHAPTER 1: Introduction..................................................... 1

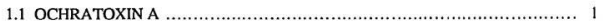

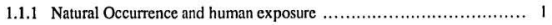

1.1.2 Stability of OTA and effects of food processing ...................... 2

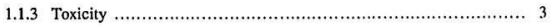

1.1.4 Teratogenicity .................................................... 4

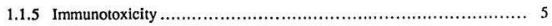

1.1.6 Carcinogenicity and mutagenicity.....................................

1.1.7 Absorption, distribution and metabolism .......................... 7 


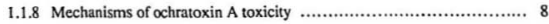

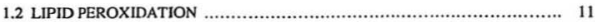

1.2 .1 Definition ............................................................ 11

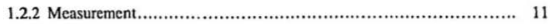

1.2.3 Enzymatic lipid peroxidation systems.................................... 12

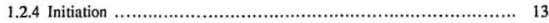

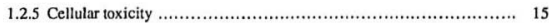

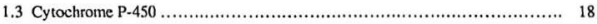

1.3.1 Background and role in xenobiotic metabolism....................... 18

I.3.2 Induction............................................................. 20

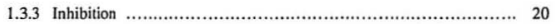

1.3.3a Reversible Inhibitors....................................... 21

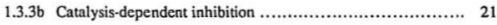

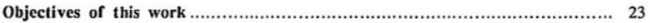

CHAPTER 2: Materials and Methods ........................................ 38

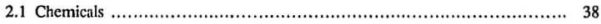

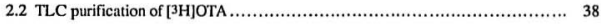

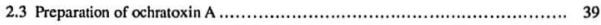

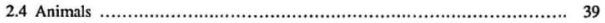

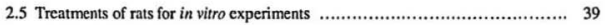


vii

2.5.1 Treatments with cytochrome P-450 inducers

2.5.2 Treatments with Cobalt protoporphyrin IX for microsomal preparations

2.6 Treatments of rats for $I n$ vivo studies

2.6.1 Inducing cytochrome P-450 40

2.6.2 [3H]OTA experiments 41

2.7 Preparation of liver microsomes 41

2.8 Cytocnrome $\mathrm{P}-450$ purification 41

2.9 Cytochrome P-450 assay 43

2.10 NADPH-cytochrome $\mathrm{P}-450$ reductase purification 44

2.11 NADPH-cytochrome P-450 reductase assay........................................ 45

2.12 Fortification of microsomes from Co-heme treated rats with Fp ................ 46

2.13 Preparation of phospholipid vesicles ...................................... 46

2.14 Lipid peroxidation assays (incubation conditions) $\ldots \ldots \ldots \ldots \ldots \ldots \ldots \ldots \ldots \ldots . . . \ldots 7$

2.14.1 Reconstituted system incubations ................................ 47

2.14.2 Microsomal incubations............................................. 47

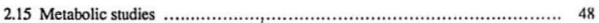

2.15.1 Metabolism of OTA In vitro ............................................ 48

2.15.2 Effect of monoclonal antibodies on OTA metabolism in vitro .......... 48

2.15.3 Analysis of OTA metabolites formed in in vitro studies................. 49

2.16 Ethoxy- and pentoxyresorufin O-dealkylation assays............................ 49

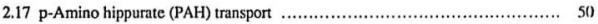

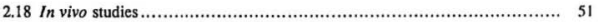

2.18.1 Urinary enzyme experiments ................................... 51

2.18.1 a Preparation of urine samples ............................... 51 
2.18.1b Gel filtration of urine samples ........................... 51

$2.18 .1 \mathrm{c}$ Measurement of urinary enzymes ......................... 51

$2.18 .2\left[{ }^{3} \mathrm{H}\right] \mathrm{OTA}$ experiments.......................................... 51

2.19 Spectrophotometric/spectrofluorometric measurements .................... 52

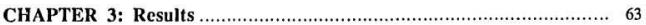

3.1 Role of EDTA and cytochrome P-450 in the stimulation of OTA-induced lipid peroxidation in reconstituted systems .........................................

3.2 Effect of microsomes from Co-heme pretreated rats on OTA-stimulated lipid peroxidation ............................................................. 64

3.3 Effect of various cytochrome P-450 inducers on OTA metabolism by liver microsomes ............................................................... 64

3.4 Substrate specificity of cytochromes P-450 IIB1 and IA $1 / 1 \mathrm{~A} 2 \ldots \ldots \ldots \ldots \ldots \ldots .65$

3.5 Effect of specific inhibitors of cytochromes P-450 IA1/IA2 and IIB1 on the metabolism of OTA in vitro

3.6 Effect of monoclonal antibodies (MAbs) against cytochromes P-450 on the metabolism of OTA by liver microsomes in vitro 66

3.7 Time course of OTA metabolism by liver microsomes from rats treated with

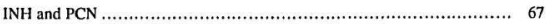

$3.8 \mathrm{Effect}$ of $\mathrm{pH}$ on OTA metabolism and lipid peroxidation ...................... 68

3.9 Effect of active oxygen scavangers, antioxidants and iron chelators on OTA metabolism and lipid peroxidation.................................................. 68

3.10 Effect of various hemoproteins and hydroperoxides on OTA metabolism ........ 70

3.11 Effect of $\mathrm{pH}$ on OTA metabolism and lipid peroxidation by liver microsomes, 
and on cytochrome P-450 content of these microsomes

3.12 Effect of $\mathrm{pH}$ on the $\mathrm{Km}$ and $\mathrm{Vmax}$ of OTA hydroxylase in liver mierosomes from rats treated with $3 \mathrm{MC}$ and ISF

3.13 In vivo experiments

3.13.1 Effect of PB treatment on OTA-induced release of enzymes in the urine

3.13.2 Effect of PB treatment on the distribution of OTA

4.1 Involvement of cytochrome P-450 in the stimulation of ochratoxin A. induced lipid peroxidation

4.2 Role of cytochrome P-450 in OTA metabolism ................................ 142

4.3 Possible mechanism of the formation of 4(S)-4-OH-OTA ....................... 146

4.4 Role of cytochrome P-450 in OTA-induced toxicity ............................. 152

CONCLUSIONS 


\section{LIST OF TABLES}

Table1 Ocurrence of OTA in food and animal feed of plant origin ............

of

Table 2 The incidence of OTA in meat and meat products

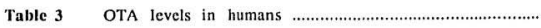

Table $4 \quad$ LD $_{50}$ of OTA in different species

Table 5 Regulation of cytochrome P-450 gene expresion

Table 6 The $R_{f}$ values of ochratoxin $A$

Table 7 List of various treatments and cytochromes P-450 induced ..........

Table 8 Involvement of EDTA or cytochrome P-450 in the stimulation

of lipid peroxidation by OTA in a reconstituted system

Table 9 Requirement for cytochrome P-450 in NADPH-dependent

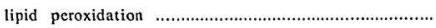

Table 10 Effect of Co-heme pretreatment on liver microsomal NADPH-cytochrome P-450 reductase and cytochrome

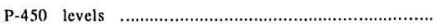


Table 11 Total cytochrome P-450 content of liver microsomes isolated from rats following various treatments

Table 12 Ethoxy-and pentoxyresorufin O-dealkylation by liver microsomes from control, $3 \mathrm{MC}$ and $\mathrm{PB}$ treated rats

Table 13 Cross reactivity of MAB 1-7-1, 2-66-3 and 2-3-2 with cytochromes $\mathrm{P}-450$ in liver microsomes isolated from rats treated with $\mathrm{PB}, 3 \mathrm{MC}$ or Dxm

Table 14 Effect of INH-and PCN-pretreatments on hepatic cytochrome P-450 levels and OTA metabolism 100

Table 15 Effect of active oxygen scavengers and antioxidant on NADPHdependent OTA metabolism and lipid peroxidation

Table 16 Effect of antioxidants and iron chelators on OTA metabolism and cytochrome P-450 content

Table 17 Effect of BHA and mannitol on ascorbate-dependent OTA metabolism and lipid peroxidation

Table 18 Ability of various hemoproteins/hydroperoxides to metabolize OTA 
xii

Table 19 Comparison of the ability of microsomes isolated from rats

treated with $3 \mathrm{MC}$, ISF and PB to metabolize OTA at pH 6.0

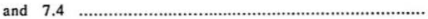

119

Table 20 The effect of $\mathrm{pH} 6.0$ and 7.4 on the ability of microsomes

isolated from rats treated with $3 \mathrm{MC}$, ISF and $\mathrm{PB}$ to undergo

lipid peroxidation and cytochrome P-450 destruction ..................

120

Table 21 Sequential homology between rat and human cytochrome P-450

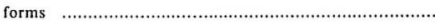




\section{xiii \\ LIST OF FIGURES}

Page

Figure 1 Structures of ochratoxin A and its metabolites

Figure 2 Factors influencing the occurrence of mycotoxins in human food

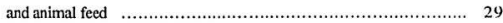

Figure 3 Simplified reactions of the process of lipid peroxidation

Figure 4 The catalytic cycle of cytochrome P-450-catalyzed reactions and OTA hydroxylation.

Figure 5 The UV spectrum of OTA. 53

Figure 6 The fluorescence spectrum of OTA. 5.5

Figure 7 The HPLC profile of our OTA preparation. 58

Figure 8 Proton NMR spectrum of my OTA preparation. 60)

Figure 9 Effect of cytochrome P-450 concentration on OTA stimulated lipid peroxidation.

Figure 10 Effect of depleting cytochrome P-450 on OTA stimulated lipid peroxidation. 
xiv

Figure 11 HPLC profile of OTA and its metabolites 4(R)- and 4(S)-4-OH-

Figure 12 The formation of $4(\mathrm{R})$ - and $4(\mathrm{~S})$-4-OH-OTA in vitro using liver microsomes from rats treated with different inducers of cytochrome P-450.

Figure 13 The formation of $4(\mathrm{R})$ - and $4(\mathrm{~S}) \cdot 4-\mathrm{OH}-\mathrm{OTA}$ in vitro using liver microsomes from rats treated with different inducers of cytochrome P.450 (Batch II)

Figure 14 The formation of $4(\mathrm{R})$ - and $4(\mathrm{~S})$-4-OH-OTA in vitro using liver microsomes from control rats treated with the vehicles of the different inducers of cytochrome P-450.

Figure 15 Effect of the cytochrome P-450 inhibitors $\alpha$-naphthoflavone (50 $\mu \mathrm{M})$ and metyrapone $(100 \mu \mathrm{M})$ on the metabolism of OTA to $4(\mathrm{R})$ 4-OH-OTA by liver microsomes from $\mathrm{PB}, 3 \mathrm{MC}$ and ISF treated rats.

Figure 16 Effect of the cytochrome P-450 inhibitors $\alpha$-naphthoflavone (50 $\mu \mathrm{M})$ and metyrapone $(100 \mu \mathrm{M})$ on the metabolism of OTA to $4(\mathrm{~S})$ 4-OH-OTA by liver microsomes from PB, 3MC and ISF treated rats. 
Figure 17a Effect of monoclonal antibody (MAb) 1-7-1 on the metabolism of OTA by microsomes from rats treated with $3 \mathrm{MC}$.

Figure $17 \mathrm{~b}$ Effect of rabbit IgG on the metabolism of OTA by microsomes from rats treated with $3 \mathrm{MC}$

Figure 18a Effect of monoclonal antibody (MAb) 2-66-3 on the metabolism of OTA by microsomes from rats treated with PB.

Figure 18b Effect of rabbit IgG on the metabolism of OTA by microsomes from PB treated rats.

Figure 19 A. Time course of OTA metabolism by liver microsomes from INHtreated rats.

B. Time course of lipid peroxidation in liver microsomes from INH-treated rats.

101

Figure 20 A. Time course of OTA metabolism by liver microsomes from PCN-treated rats.

B. Time course of lipid peroxidation in liver microsomes from $\mathrm{PCN}$-treated rats.

Figure 21 Effect of $\mathrm{pH}$ on OTA metabolism (A) and lipid peroxidation (B) by liver microsomes from INH-treated rats. 
xvi

Figure 22 Effect of $\mathrm{pH}$ on OTA metabolism (A) and lipid peroxidation (B) by liver microsomes from PCN-treated rats.

Figure 23 Effect of varying BHA concentrations on (A) OTA matabolism and (B) lipid peroxidation by liver microsomes from INH-treated rats. ...

Figure 24 Effect of $\mathrm{pH}$ on OTA metabolism (A), and lipid peroxidation (B) by

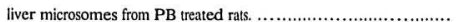

Figure 25 Effect of $\mathrm{pH}$ on OTA metabolism by liver microsomes from $3 \mathrm{MC}$ treated rats.

Figure 26 Effect of $\mathrm{pH}$ on the $\mathrm{Km}$ and Vmax of OTA hydroxylation by liver microsomes from $3 \mathrm{MC}$ treated rats.

Figure 27 Effect of $\mathrm{pH}$ on the $\mathrm{Km}$ and $\mathrm{Vmax}$ of OTA hydroxylation by liver microsomes from ISF treated rats...............................

Figure 28 Alkaline phosphatase activity in urines of control and PB-treated rats following OTA administration.

Figure 298 -Glutamyl transferase activity in urines of control and PB-treated rats following OTA administration.

127

Figure 30 [14C]PAH transport in tenal cortex slices from control (C), OTA treated (OTA) and PB-pretreated treated with OTA (PB + OTA) rats. . 
xvii

Figure $31 \quad\left[{ }^{3} \mathrm{H}\right] \mathrm{OTA}$ levels in serum and urine of control and PB-treated rats 6 hours after one oral treatment with [3H]OTA $(288 \mu \mathrm{g} / \mathrm{kg}$ in $50 \mathrm{mM}$ $\mathrm{NaHCO}$

Figure 32 [3H]OTA levels in liver and kidney of control and PB-treated rats 6 hours after one oral treatment with [3H]OTA $(288 \mu \mathrm{g} / \mathrm{kg}$ in $50 \mathrm{mM}$ $\mathrm{NaHCO}$

Figure $33\left[{ }^{3} \mathrm{H}\right] \mathrm{OTA}$ levels in the gut of control and PB-treated rats 6 hours after one oral treatment with [3H]OTA $(288 \mu \mathrm{g} / \mathrm{kg}$ in $50 \mathrm{mM}$ $\mathrm{NaHCO}$ )

Figure 34 [3H]OTA levels in the gut contents of control and PB-treated rats 6 hours after one oral treatment with [ ${ }^{3 \mathrm{H}}$ ]OTA $(288 \mu \mathrm{g} / \mathrm{kg}$ in $50 \mathrm{mM}$ $\mathrm{NaHCO}_{3}$

Figure 35 Scheme representing the possible mechanism of $4(\mathrm{R})$ - and $4(\mathrm{~S})-4$ OH-OTA formation and of OTA-stimulated lipid peroxidation. 
xviii

\section{LIST OF A BBREVIATIONS}

$\begin{array}{ll}\text { BEN, } & \text { Balkan endemic nephropathy } \\ \text { BHA, } & \text { butylated hydroxyanisole } \\ \text { BHT, } & \text { Butylated hydroxytoluene } \\ \text { BNF, } & \text { B-naphthoflavone } \\ \text { BPS, } & \text { bathophenanthrolinedisulfonic acid } \\ \text { C, } & \text { control (no treatment) } \\ \text { CHAPS, } & \text { 3-I(cholamidopropyl)-dimethylammonio]-1-propanesulfonate } \\ \text { CHP, } & \text { cumene hydroperoxide } \\ \text { CLF, } & \text { clofibrate } \\ \text { DPPD, } & \text { N,N-diphenyl-1,4-phenylenediamine } \\ \text { DTT, } & \text { dithiothreitol } \\ \text { FMN, } & \text { flavin mononucleotide } \\ \text { Fp, } & \text { NADPH-cytochrome P-450 reductase } \\ \text { HRP, } & \text { horseradish peroxidase } \\ \text { INH, } & \text { isoniazid } \\ \text { ISF, } & \text { isosafrole } \\ \text { LAHP, } & \text { linoleic acid hydroperoxide } \\ \text { MAb, } & \text { monoclonal antibody } \\ \text { 3MC, } & \text { 3-methylcolanthrene } \\ \text { MDA, } & \text { malondialdehyde } \\ \text { OTA, } & \text { ochritoxin A } \\ \text { PB, } & \text { sodium phenobarbital } \\ \text { PCN. } & \text { pregnenolone-16o-carbonitrile } \\ \text { SOD, } & \text { superoxide dismutase } \\ & \end{array}$


xix

TBA, 2-thiobarbituric acid

TCA, trichloroacetic acid.

$\mathrm{v} / \mathrm{v}, \quad$ volume/volume

w/v, weight/volume

NOTE - For the sake of brevity, in some tables and figures, the term INH microsomes, PCN microsomes or Co-heme microsomes. This is meant to imply that the microsomes were isolated from the livers of INH-, PCN- or cobalt protoporphyrin IX (Co-heme)pretreated rats and not that the microsomes contained INH, PCN or Co-heme. 


\section{CHAPTER 1}

\section{INTRODUCTION}

\subsection{OCIIRATOXIN A}

\subsubsection{Natural Occurrence and human exposure}

Ochratoxin A (OTA) is a mycotoxin produced by some species of the fungal genera Aspergillus and Penicillium. It was first reported in 1965 by De Scott (1965) as the toxic metabolite in a culture medium of Aspergillus ochraceus wilh. OTA was chemically characterized by Van der Merwe et al. (1965a,b) and Steyn and Holzapfel (1967). It consists of a 5'-chloro-8-hydroxy-3,4-dihydro-3-methyl isocoumarin moiety linked by an amide bond to L-B-phenylalanine (Figure 1). The toxigenic molds known to produce the toxin are the following species:

Aspergillus species Penicillium species
A. ochraceus
P. viridicatum
A. ostianus
P. cyclopium
A. melleus
P. commune
A. petrakii
R. palitans
A. sclerotionm
D. purpurescens
A. sulphureus
R. variabile

The occurrence of OTA in food and feed is widespread (Krogh and Nesheim, 1982). It is present as a contaminant in plant products, especially, cereals, beans and peanuts (Kurata, 1978). OTA is also found in meat, dried fish (Ueno, 1985a) as well as in the kidneys, liver and blood of slaughtered pigs (Hult et al., 1980). Scott et al. (1972) detected OTA in concentrations of up to $27 \mu \mathrm{g} / \mathrm{gm}$ in 18 out of 29 samples of heated grain from Saskatchewan farms in Canada. In a recent analysis of 1200 blood samples obtained from pigs slaughtered in Westem Canada, Marquardt et al. (1988) found that $76 \%$ of the samples had detectable levels of OTA and $11.3 \%$ had OTA levels of $>10 \mathrm{ng} / \mathrm{mL}$ with the 
highest being $229 \mathrm{ng} / \mathrm{mL}$. The World Health Organization (WHO) recently reviewed OTA. and in their report they summarized the occurrence of OTA in food and animal feed of plant origin around the world (WHO, 1990). Among the highest levels of OTA were those samples listed in Table 1.

Residues of OTA are not generally found in ruminants, because OTA is cleaved in their forestomachs by protozoan and bacterial enzymes (Galtier and Alvinerie, 1976; Hult et al., 1976); the non-toxic hydrolytic cleavage product is ochratoxin a (ochratoxin A without the phenylalanine moiety). When two milking cows were fed a ration containing $317-1125$ $\mu \mathrm{g}$ OTA $/ \mathrm{kg}$ body weight for 11 weeks, a residue of $5 \mu \mathrm{g}$ OTA $/ \mathrm{kg}$ was found in the kidneys of one of the animals but not in any other tissue or the milk. Ochratoxin a was not found in any tissue (Shreeve et al., 1979). Residues of OTA have been detected in a number of tissues in single-stomached animals such as pigs (Hult et al., 1980). OTA is also present in the blood because it binds to serum albumin (Chu, 1971; Galtier, 1974). The incidence of OTA in meat and meat products has been reviewed by Marquardt et al. (1990) (Table 2).

Human exposure occurs through the direct consumption of contaminated food (mainly cereals) and/or meat from animals that retain OTA in their tissues after being fed contaminated feed (see diagram, Figure 2), OTA levels in humans were reported in a review by Kuiper-Goodman and Scott (1989) (Table 3).

\subsubsection{Stability of OTA and effects of food processing}

OTA is moderately stable, even on heating, in such food as wheat, pork and beans. However, levels of OTA added to ground green coffee bcans were reduced $87 \%$ on roasting for $20 \mathrm{~min}$ at $200^{\circ} \mathrm{C}$ (Levi et al., 1974). Tsubouchi et al. (1987) found that after inoculation with $\mathrm{A}$ ochraceus, roasting green coffee beans for $20 \mathrm{~min}$ at $200^{\circ} \mathrm{C}$ reduced the concentration of OTA by a maximum of only $12 \%$ in whole beans and $18-22 \%$ in ground beans. They concluded that the mode of OTA contamination was the reason for this greater 
heat resistance. Cleaning and milling of wheat and barley did not remove OTA in naturally contaminated samples, and levels in flour and bran were the same (Chelkowski et al., 1981). There was, however, a marked reduction of OTA levels in pearled barley compared to those in bran (Chelkowski et al., 1981). Cooking of polished wheat by an Egyptian method destroyed only $6 \%$ of added OTA, and during cooking of faba beans by two methods the average destruction was $16 \%$ and $20 \%$ (El-Banna and Scott, 1984).

\subsubsection{Toxicity}

OTA is known to be highly toxic to animals (Van der Merwe et al., 1965 a; Purchase and Theron, 1968; Peckham et al, 1971 and Szczech et al,, 1973). The main pathological changes associated with OTA toxicity are primarily damage to the kidney and to a lesser extent damage to the liver (Harwig, 1974 and Krogh, 1978). Alterations in a variety of biochemical and immunological parameters have also been observed following OTA administration (Berndt et al., 1980; Meisner and Meisner, 1981; Haubeck et al., 1981; and Kane et al., 1986). The changes in renal function in OTA-exposed rats and pigs are characterized by polyuria, glucosuria, proteinuria and an increase in blood urea nitrogen, and a decrease in urine osmolarity and glomerular filtration rate (Berndt et al., 1980). Kane et al. (1986) observed a good correlation between the increase in urinary excretion of glutamyl transferase, alkaline phosphatase and leucine aminopeptidase and a decrease in renal activities of these same enzymes within a week of the oral administration of $145 \mu \mathrm{g}$ OTA $/ \mathrm{kg}$ body weight for 12 weeks.

An inhibition of renal gluconeogenesis was also observed in kidney-cortex slices from rats which had been fed with $2 \mathrm{mg}$ OTA/kg body weight for 2 days (Meisner and Selanik, 1979) most probably because renal phosphoenolpyruvate carboxykinase was inhibited (Meisner and Meisner, 1981). After the daily administration of OTA (5 mg/kg) for 3 days to rats, Suzuki et al. (1975) observed a $60 \%$ decrease in hepatic glycogen levels 
and a concomitant increase in serum glucose, and blood and liver lactate levels. Later, Subramanian et al. (1989) showed that OTA drastically depleted liver glycogen, increased blood glucose level and lowered serum insulin level in rats. The concluded that OTA is diabetogenic in nature, and exerts its effect by reducing the level of insulin, thereby suppressing glycogenesis and enhancing glycogenolysis. Administration of OTA to mice inhibited protein synthesis, the degree of inhibition $5 \mathrm{hr}$ after administration of $1 \mathrm{mg}$ OTA $/ \mathrm{kg}$ body weight being $26 \%$ in liver, $68 \%$ in kidney and $75 \%$ in spleen (Creppy et al., 1984). Phenylalanine ( $100 \mathrm{mg} / \mathrm{kg}$ body weight) injected together with OTA (10 mg/kg body weight) prevented the inhibition of protein synthesis in all of these organs (Creppy et al., 1984). OTA is thought to inhibit protein synthesis through its competition with phenylalanine in the reaction catalyzed by phenylalanyl t-RNA synthetase (Creppy et al., 1984). Interestingly, babies born with phenylketonuria are less susceptible to OTA toxicity because of their elevated level of phenylalanine (Woolf, 1986). OTA was shown to inhibit rat liver mitochondrial respiration by acting as a competitive inhibitor of carrier proteins located in the inner mitochondrial meinbrane (Meisner and Chan, 1974; Wei ct al., 1985). The $\mathrm{LD}_{50}$ values of OTA in various species are listed in Table 4 (WHO, 199()).

\subsubsection{Teratogenicity}

OTA is a potent teratogen in mice, rats and hamsters. OTA injected intraperitoneally in pregnant mice at $5 \mathrm{mg} / \mathrm{kg}$ body weight on one of gestation days $7-12$ resulted in increased prenatal mortality, decreased fetal weight, and various malformations, including exencephaly and anomalies of the eyes, face, digits and tail (Hayes et al., 1974). When rats were treated orally with OTA at $0.25,0.50,0.75,1,2,4$ or $8 \mathrm{mg} / \mathrm{kg}$ body weight on gestation days 6-15, maternal toxicity was not observed below $4 \mathrm{mg} / \mathrm{kg}$ body weight, but an increased incidence of fetal resorptions was observed above $0.50 \mathrm{mg} / \mathrm{kg}$ body weight (Brown et al., 1976). Subcutaneous administration of OTA $(1.75 \mathrm{mg} / \mathrm{kg}$ 
body weight) on gestation days 5-7 resulted in the highest number of malformations, including hydrocephaly, omphalocele and anophthalmia, as well as a shift in position of the oesophagus (Mayura et al., 1982). Increased prenatal mortality and malformations, including hydrocephaly, micrognathia, and heart defects were observed in hamsters injected intraperitoneally with OTA at doses of $5-20 \mathrm{mg} / \mathrm{kg}$ body weight on one of gestation days 7-9 (Hood et al., 1976).

\subsubsection{Immunotoxicity}

In chickens fed 2-4 ppm OTA in the diet for 20 days there was a depression of IgG, IgA and IgM in lymphoid tissues and serum (Dwivedi and Burns, 1984). OTA in the concentration range studied $(20.1667 \mu \mathrm{mol} / \mathrm{L})$ caused a $47-50 \%$ inhibition of macrophage migration (Klinkert et al., 1981); this effect could be prevented by the simultaneous addition of phenylalanine to the medium. In Balb/c mice, a dose of OTA as low as 0.005 $\mu \mathrm{g} / \mathrm{kg}$ was able to suppress the immune response to sheep red blood cells (Haubeck et al., 1981). In the standard plaque counting assay for the estimation of antibody-producing spleen lymphocytes OTA ( $1 \mu \mathrm{g} / \mathrm{kg}$ body weight) given once intraperitoneally to Balb/c mice, 8-12 weeks of age, had an immunosuppressive effect on both IgM and IgG responses to a single injection of sheep red blood cells (Creppy et al., 1982). The immunosuppressive effects of OTA could be prevented by the intraperitoneal administration of phenylalanine at $10 \mu \mathrm{g} / \mathrm{kg}$ body weight (Haubeck et al., 1981 and Creppy et al., 1982). Thus, the immunosuppressive action of OTA could be due to its action on protein synthesis. When female $\mathrm{B} 6 \mathrm{C} 3 \mathrm{Fl}$ mice were administered ochratoxin $\mathrm{A} 6$ times during 12 days in amounts of $0.34,6.7$, or $13.4 \mathrm{mg} / \mathrm{kg}$ body weight (total dosage), the natural killer cell activity was inhibited at all dose levels, and the growth of transplantable tumour cells was increased without affecting T-cell or macrophage-mediated antitumour activity. Ochratoxin B did not influence immune function at $13.4 \mathrm{mg} / \mathrm{kg}$ body weight administered 6 
times during 6 days (Luster et al., 1987). The inhibition by OTA of nitural killer cell activity appeared to be caused by reduced production of basal interferon. Because natural killer cells are involved in the destruction of tumour cells, the ability of OTA to modulate the activity of these cells might contribute to its capacity to induce renal and hepatic carcinomas. Lea et al. (1989) reported that OTA has a potent inhibitory effect both at the level of B and T lymphocytes.

\subsubsection{Carcinogenicity and mutagenicity}

Kanisawa and Suzuki (1978) reported renal and hepatic tumors in male ddY mice after they were fed a diet containing $40 \mathrm{mg}$ OTA/kg for 45 weeks. Dietary feeding of OTA (40) $\mathrm{mg} / \mathrm{kg}$ diet for 20 months) has also been shown to induce renal adenomas and hepatocellular carcinomas in mice (Bendele et al., 1985). It was quoted that ochratoxin A is one of the most potent toxic carcinogens ever tested by the National Toxicology Progrum (Dietrich and Swenberg, 1993). In that extensive carcinogenicity study by the National Toxicology Program, OTA administered by gastric intubation in corn oil at levels of 70 and $210 \mu \mathrm{g} / \mathrm{kg}$ body wcight/day for 24 months induced high incidences of renal cell carcinoma of a clearly malignant nature in rats (Boorman, 1988). OTA has also been associated with Balkan endemic nephropathy (BEN), a disease that affects residents of certain regions of Bulgaria, Romania and Yugoslavia where OTA contaminates their crops (Krogh, 1974). In these infected areas, OTA was isolated from the sera of residents and those who had high OTA levels also (often) had urinary tract tumors and BEN (Hult et al., 1982a,b and Petkova-Bocharova et al., 1988). Creppy et al.(1985) showed that OTA administration induced single strand breaks in DNA in liver, kidney and spleen, and they suggested that OTA is weakly genotoxic to mammalian cells. OTA has been tested for mutagenicity in a variety of Salmonella typhimurium strains, with and without metabolic activation, and

results were negative (Wehner et al., 1978; Bartsch et al., 1980). Recently, after 
incubation with cultured hepatocytes for 24 hours, OTA was shown to be mutagenic to Salmonella typhimurium in a cell-free culture medium (Hennig et al., 1991).

\subsubsection{Absorption, distribution and metabolism}

Small intestine is thought to be the primary site of ochratoxin A absorption. When OTA was injected into the lumen of the stomach, the small intestine, cecum or colon of male Wistar rats, the highest absorption was in the proximal jejunum (Kumagai and Aibara, 1982). When OTA was given orally to mice, the site of highest absorption was the duodenum (Lee et al., 1984). In the latter study, immunohistochemical staining revealed that the highest concentration of OTA was in the intestine with decreasing levels in the kidney and liver (Lee et al., 1984).

Galtier et al. (1979) studied the pharmacokinetic profile of ochratoxin A using [14C]OTA injected intravenously to rats. Six hours after injection, $24 \%$ of the administered dose was in the plasma, $12 \%$ in muscle, $11.1 \%$ in skin, $2.63 \%$ in the liver and $0.64 \%$ in the kidneys. Per gram of tissue, the concentration of OTA was higher in the kidneys than in the liver. The stomach, small intestine, cecum and large intestine contained $0.34 \%$, $2.14 \%, 0.66 \%$ and $0.57 \%$ of the administered dose, respectively. The contents of the stomach, small intestine, cecum and large intestine contained $0.33 \%, 4.02 \%, 4.02 \%$ and $1.87 \%$ of the administered dose respectively.

A number of studies have shown that, in the presence of NADPH, OTA is metabolized by liver microsomes from several species (rat, pig and human) primarily to 4(R)-4-OH-OTA, and to a smaller extent to the epimeric 4(S)-4-OH-OTA (Stormer et al., 1981, 1983; Ueno, 1985; Oster et al., 1991; Hietanen et al., 1986). OTA metabolism has also been shown to be induced by PB and/or 3MC in Wistar rats (Stormer and Pedersen, 1980), DA and Lewis rats (Hietanen et al., 1986) and in rats of unidentified strain (Jeno, 1985). 


\subsubsection{Mechanisms of ochratoxin A toxicity}

The mechanism by which ochratoxin A exerts its toxic effects is not known yet. In a recent review by Marquardt and Frohlich (1992), three potential mechanisms of ochratoxicosis were recognizad which involved effects of OTA on the enzymes involved in phenylslanine metabolim, effects on lipid peroxidation, and effects on mitochondrial function.

Ochratoxin A affects DNA, RNA, and protein synthesis in many different organisms (Meisner and Meisner, 1981; Creppy et al., 1983a,b, 1986; Meisner et al., 1983; Roschenthaler et al., 1984; Meisner and Krogh, 1986; Meisner and Polsinclli, 1986), presumably due to an effect by the phenylalanine moiety of OTA. Administration of OTA to mice inhibited protein synthesis; the degree of inhibition $5 \mathrm{hr}$ after administration of $1 \mathrm{mg}$ OTA $/ \mathrm{kg}$ body weight was $26 \%$ in liver, $68 \%$ in kidney and $75 \%$ in spleen (Creppy et al., 1984). Phenylalanine (100 $\mathrm{mg} / \mathrm{kg}$ body weight) injected together with ochratoxin $\mathrm{A}$ (10 $\mathrm{mg} / \mathrm{kg}$ body weight) prevented the inhibition of protein synthesis in all of these organs (Creppy et al., 1984). OTA is thought to inhibit protein synthesis through competition with phenylalanine in the reaction catalyzed by phenylalanyl t-RNA synthetase. OTA also inhibited phenylalanine hydroxylase (Creppy et al., 1990). Ochratoxin a (OTA without the phenylalanine moiety), in contrast, was not effective, which indicates the importanec of phenylalanine for the inhibition. The blockage of phenylalanine hydroxylase activity could produce phenylketonuria-like effects, including an enhanced production of phenylpyruvate and phenylacetate (Creppy et al., 1990)

Rahimtula et al. (1988) have demonstrated that when administered to rats in vivo or when added to liver or kidney microsomes in vitro, OTA greatly enhanced lipid peroxidation (Rahimtula et al., 1988). In a subsequent study (Omar et al., 1990) using a reconstituted microsomal lipid peroxidation system consisting of microsomal phospholipid, the flavoprotein NADPH cytochrome P-450 reductase (Fp) and iron ions, we demonstrated 
that OTA induced lipid peroxidation by chelating $\mathrm{Fe}^{3+}$, that the resulting OTA- $\mathrm{Fe}^{3+}$ chelate was readily reducible by the flavoprotein NADPH cytochrome P-450 reductase to the OTA-Fe $2+$ complex. The latter in the presence of oxygen provided the active species that initiated lipid peroxidation. Using electron spin resonance, the production of the extremely toxic hydroxyl radicals by the $\mathrm{Fe}^{3+}$ complex of OTA in the presence of the NADPHcytochrome P-450 reductase system was also demonstrated (Hasinoff et al., 1990). Khan et al. (1989) have shown that OTA-stimulated lipid peroxidation is also accompanied by the leakage of calcium from calcium-loaded microsomes. Lipid peroxidation may be an important process in hepatotoxicity, which results in structural changes in the cell membrane sufficient to allow an influx of extracellular calcium to cause changes in metabolic activity within the cell (Orrenius and Bellomo, 1986). Aleo et al. (1991) have reported that OTA stimulated lipid peroxidation in isolated rat renal proximal tubules. Because mitochondrial toxicity was not changed by the iron chelator desferal, they suggested that OTA toxicity in the proximal tubules (indicated by increased lactate dehydrogenase release and decreased ATP production) was primarily related to inhibition of mitochondrial function and not to iron-mediated lipid peroxidation. OTA toxicity to the mitochondria is discussed further below. The latter study, however, did not conclusively rule out the importance of lipid peroxidation, and the role of lipid peroxidation in the toxicity of OTA remains to be determined.

Ochratoxin A has been shown to inhibit the respiration of whole mitochondria by acting as a competitive inhibitor of carrier proteins located in the inner mitochondrial membrane (Meisner and Chan, 1974; Wei et al., 1985). Meisner (1976) has shown that the uptake of OTA by mitochondria resulted in intramitochondrial ATP depletion. He also showed that OTA inhibited intramitochondrial phosphate transport, resulting in deterioration of the mitochondria. The failure of OTA to deplete ATP in rat hepatocytes 
observed by Creppy et al. (1990), and the ability of $0 \alpha$ which is non-toxic, to inhibit mitochondrial respiration more effectively than OTA (Moore and Truelove, 1970) would suggest that mitochondrial effects probably did not contribute to the toxic effects of OTA. 


\subsection{LIPID PEROXIDATION}

\subsubsection{Definition}

In eukaryotes, membrane fluidity is maintained by the incorporation of polyunsaturated fatty acid (PUFA) chains into membrane lipids. Most of these PUFA chains occur on the 2-C position of the glycerol moiety of phospholipids, particularly phosphatidylcholine and phosphatidylethanolamine, although some also occur in neutral lipids. In the membranes of rat liver microsomes, the most abundant PUFAs (expressed as percentage of total fatty acids) are: arachidonic acid (17\%), linoleic acid (10\%), docosahexanoic acid (5\%) and linolenic acid (5\%) (Horton and Fairhurst, 1987). In these acids the presence of an adjacent double bond weakens the carbon-hydrogen bonds. These allylic hydrogens can be abstracted by reactive species containing one or more unpaired electrons (free radicals). The lipid radical thus formed will then react with molecular oxygen, and the ensuing chain reaction results in the breakdown of the PUFA. This reaction sequence is known as lipid peroxidation. Lipid peroxidation propagates by reaction between a radical molecule and a non-radical molecule and terminates when two radicals react with each other. The reactions of lipid peroxidation may be categorized into three main steps: 1) initiation, 2) propagation and 3) termination (Figure 3).

\subsubsection{Measurement}

The products of lipid peroxidation include lipid epoxides, hydroperoxides, epoxy alcohols, and the short-chain compounds such as malondialdehyde (MDA), ethane, pentane, and 4-hydroxy alkenals (Dahle et al., 1962; Dillard et al., 1977; Hafeman and Hoekstra, 1977: Benedetti et al., 1980). Lipid peroxidation has been measured by the detection of conjugated dienes formed during the early phase of the peroxidation reaction sequence (Corongiu and Milia, 1983; Recknagel and Glende, 1984), and less commonly 
by measurement of lipid hydroperoxides (Catheart et al., 1983; Pryor and Castle, 1984). The most common procedures are based on the measurement of the products of lipid hydroperoxide breakdown such as malondialdehyde (MDA). This is the most widely used method because of its simplicity and sensitivity, and MDA has been commonly detected by the thiobarbituric acid (TBA) reaction (Niehaus and Samuelsson, 1968; May and McCay, 1968; Frankel and Neff, 1983; Csallany et al., 1984). In addition, lipid peroxidation has been assayed recently by the evolution of short-chain alkanes (ethane and pentane) both in vivo and in vitro (Lawrence and Cohen, 1982; Kappus and Muliawan, 1982; Kunert and Tappel, 1983; Muller and Sies, 1984).

\subsubsection{Enzymatic lipid peroxidation systems}

Enzymatically induced microsomal lipid peroxidation was first described by Hochstein and Ernster in 1963, who demonstrated the requirement for reduced nicotinamide adenine dinucleotide phosphate (NADPH) and adenosine 5 '-diphosphate (ADP) and the enzymatic nature of the process (Hochstein and Ernster, 1963). In a subsequent study, they showed the necessity for iron (Hochstein et al., 1964) which had been a contaminant of their original ADP solutions. Later, Pederson and Aust (1972) characterized the enzymatic nature of lipid peroxidation further by demonstrating that NADPH-cytochrome P-450 reductase (Fp) was the enzyme linking NADPH oxidation to the ADP-Fe ${ }^{3+-d e p e n d e n t ~ p e r o x i d a t i o n ~ o f ~ m i c r o s o m a l ~ m e m b r a n e s ~(P e d e r s o n ~ a n d ~ A u s t, ~}$ 1972). They also developed a reconstituted lipid peroxidation system consisting of phospholipid vesicles (liposomes), purified Fp, ferric chelates and NADPH. In their reconstituted system, a second ferric chelate, EDTA-Fe ${ }^{3+}$ (in addition to $\mathrm{ADP}-\mathrm{Fe}^{3+}$ ) was also required (Pederson and Aust, 1972). Therefore, they suggested that there may be a microsomal component(s) that directly reduces ADP-Fe ${ }^{3+}$ (for which EDTA-Fe ${ }^{3+}$ can 
substitute) in the reconstituted lipid peroxidation system. Later, Morehouse and Aust (1988) demonstrated that when cytochrome P-450 was incorporated into phospholipid yesicles, EDTA. Fe ${ }^{3+}$ was not required suggesting that cytochrome P-450 may be the endogenous component replacing EDTA-Fe ${ }^{3+}$.

\subsubsection{Initiation}

Lipid peroxidation is sometimes a major cause of cellular injury in organisms subjected to oxidative stress (reviewed Comporti, 1985; Sies, 1985; Poli et al., 1987). Surprisingly little is known about the chemistry of initiation of peroxidation in membrane systems such as liposomes or microsomes, and the nature of the free radical species responsible for the initiation of iron-der endent lipid peroxidation has been the subject of considerable debate. The principal candidates suggested for this role are the hydroxyl radical $(\cdot \mathrm{OH})$ and the ferrous dioxygen complex $\left(\mathrm{Fe}^{2+-}-\mathrm{O}_{2}\right.$, perferryl ion). The hydroxyl radical is an extremely reactive species, reacting very rapidly with most organic molecules. Hydroxyl radicals can be formed via the Fenton reaction as follows:

$\mathrm{Fe}^{2+}+\mathrm{H}_{2} \mathrm{O}_{2} \rightarrow-\cdots \mathrm{Fe}^{3+}+\mathrm{OH}^{-}+. \mathrm{OH}$

The superoxide radical $\left(\mathrm{O}_{2}{ }^{-}\right)$is produced at a number of intracellular sites (Fridovich, 1974; Mishin et al., 1976; Chance et al., 1979) and $\mathrm{H}_{2} \mathrm{O}_{2}$ can then be formed readily from the nonenzymic or superoxide dismutase-catalyzed dismutation of $\mathrm{O}_{2}{ }^{*}$ (Chance et al, 1979). Subsequently, chelated iron can yield. $\mathrm{OH}$ in the Fenton reaction as above. An altemative hypothesis is that ferrous ion, in undergoing autoxidation to ferric ion, passes through $\mathrm{Fe}^{2+.-\mathrm{O}_{2}}$ and $\mathrm{Fe}^{3+--\mathrm{O}_{2} \cdot *}$ as intermediates. Upon the discovery of ADP-Fe3+initiated microsomal lipid peroxidation, Huchstein and Ernster (1964) postulated the 
involvement of an ADP-Fe2+-.- $\mathrm{O}_{2}$ complex. Aust's group has also pursued the idea that an ADP-ferrous dioxygen complex initiates lipid peroxidation (Svingen et al., 1978; Aust and Svingen, 1982). The involvement of a ferrous dioxygen complex has been criticized on the grounds that the complex is insufficiently reactive towards PUFA (Halliwell and Gutteridge, 1985). The highly reactive hydroxyl radical etn often be detected in microsomal or liposomal lipid peroxidation systems (Gutteridge, 1982; Morehouse et al., 1983; Gutteridge, 1984; Beloqui and Cederbaum, 1986). The hydroxyl radical is known to be capable of initiating lipid peroxidation by abstracting a hydrogen atom from fatty acids (Barber and Thomas, 1978; O'Connell and Garner, 1983). $\mathrm{H}_{2} \mathrm{O}_{2}$-degrading enzymes or scavengers of $\mathrm{OH}$, however, rarely inhibit iron-dependent peroxidation in microsomal or liposomal systems (Gutteridge, 1982; Morehouse et al., 1983; Gutteridge, 1984; Beloqui and Cederbaum, 1986). It has been proposed that the ferryl ion (sce below) is the true Fenton reagent, rather than. $\mathrm{OH}$, and this would not be scavenged by conventional. $\mathrm{OH}$ scavengers (Fee, 1981; Koppenol, 1981).

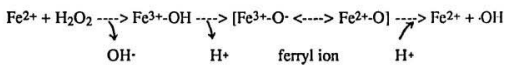

Supcroxide radicals may play a minor role in initiating lipid peroxidation under conditions in which they act to reduce $\mathrm{Fe}^{3+}$ to $\mathrm{Fe}^{2+}$ (Gutteridge 1977; Tien et al., 1981). Recently, Minotti and Aust (Minotti and Aust, 1987a and b) have proposed that a specific Fe2t-O $\mathrm{Fe}^{3+}$ complex, or at least a $1: 1$ ratio of $\mathrm{Fe}^{2+}$ to $\mathrm{Fe}^{3+}$, acts as an initiator of peroxidation in liposomal and microsomal systems, but some doubts have been raised about this complex as a specific initiator of peroxidation. Attempts to isolate such a complex have failed (Minotti and Aust, 1987a; Aust, 1988). The $\mathrm{Fe}^{2+} / \mathrm{Fe}^{3+}$ ratios required for maximal 
stimulation of peroxidation have been reported to vary from 1:1 to $1: 7$ in different experiments (Braughler et al., 1986), perhaps suggesting that a specific stoichiometric complex is not required. It must be concluded that the identity of the initiating species of lipid peroxidation produced by ferrous iron is still an open question.

\subsubsection{Cellular toxicity}

Membrane lipid peroxidation is an important part of oxidative tissue injury and can be an effect as well as a cause of reactions culminating in cytotoxicity (Halliwell and Gutteridge, 1986). The peroxidative breakdown of PUFA has been implicated in the pathogenesis of many types of injury and especially in the hepatic damage induced by several toxic substances. Among these toxic substances are the haloalkanes, carbon tetrachloride (Comporti, 1965; Slater, 1972), trichlorobromomethane (Slater, 1972; Slater, 1982), chloroform (Ekstrom and Hogberg, 1980), 1,2-dibromomethane (Albano et al., 1984) and halothane (Tomasi et al., 1983). In addition, paracetamol (Wendel and Feuerstein, 1981), bromobenzene (Casini et ql., 1985), iron (Halliwell, 1981), bipyridyl compounds (Sandy et al., 1986), allyl alcohol (Jaeschke et al., 1987) and in some instances, ethanol (Dianzani and Torrielli, 1981; Sies, 1985; Dianzani, 1985) have been shown to stimulate lipid peroxidation. The peroxidation of PUFA within biological membranes results in a complex series of biochemical and biophysical events which lead to inactivation of enzymatic functions in several subcellular organelles (Comporti, 1965; Slater, 1972; Dianzani and Ugazio, 1978; Vladimirov et al., 1980; Recknagel et al., 1982). These alterations include changes in the physical properties of the lipid bilayer, reactions between acylperoxyl radicals and membrane proteins, and the formation of reactive products originating from the degradation of peroxidized fatty acids (Dianzani and Ugazio, 1978; Vladimirov et al., 1980; Recknagel et al., 1982).

The stimulation of lipid peroxidation in either artificial membranes of liposomes or 
in subcellular organelles has been shown to increase membrane rigidity (Curtis et al., 1984; Bruch and Thayer, 1983). Such a loss of fluidity does not seem to be dependent upon an increase in the ratio between cholesterol and phospholipids (Curtis et al., 1984), but is rather an effect of the formation of cross-linking between acyl chains (Eichenberg et al., 1982) and of the depletion of long chain PUFA (Curtis et al., 1984). In addition to the changes in fluidity, lipid peroxidation causes an increase in the ionic permeability, and affects the surface potentials of the membranes (Vladimirov et al., 1980). In the liver, the membranes of the mitochondria and endoplasmic reticulum contain unsaturated fatty acids in high proportion, and therefore are vulnerable to peroxidative attack. At the same time they contain enzymes of the electron transport systems which make them capable of producing free radical species (Comporti, 1965; Slater, 1972; Vladimirov et al., 1980; Recknagel et al., 1982). The consequences for the cell of lipid peroxidation reactions and products are many. Microsomal membranes undergoing peroxidation in vitro show fragmentation and turbidity changes, destruction of cytochrome P-450 (Hogberg et al., 1973), and loss of latency and activity of glucose-6-phosphate and UDP-glucuronyl transferase (Hogberg et al., 1973; Ferrali et al., 1980; de Groot et al., 1985). The plasma membrane $\mathrm{Ca}^{2+-A}$ TPase is inactivated because of the oxidation of essential sulfhydryl groups in the enzyme (Jones et al., 1983), resulting in defective control of cytosolic calcium. Ribosomes become detached from the endoplasmic reticulum during lipid peroxidation (Palmer et al., 1978). In mitochondria, peroxidation causes membrane swelling, deterioration of electron transport, and organelle lysis (Hunter et al., 1963; Narabayashi et al., 1982). Lipid peroxidation of lysosomes causes lysis and enzyme release (Wills and Wilkinson, 1966; Fong et al., 1973), and the erythrocyte plasma membrane responds in a similar manner (Brownlee et al., 1977).

Like OTA, cephaloridine, a beta-lactam antibiotic of the cephalosporin type, causes renal injury in man and in animals, affecting the proximal tubules (Atkinson et al., 1966; 
Silverblatt et al., 1970). Of the several biochemical mechanisms proposed to explain the nephrotoxic effects of cephaloridine (Tune and Fravert, 1980; Kuo et al., 1983; Goldstein et al., 1986), the most recent hypothesis suggests an involvement of lipid peroxidation (Kuo et al., 1983; Cojocel et al., 1985a). Furthermore, recent studies showed that the formation of cephaloridine-induced reactive oxygen species and peroxidation of renal cortical membrane lipids was inhibited by radical scavengers and antioxidants (Cojocel, 1985b; Goldstein et al., 1986). 


\subsection{Cytochrome P.450}

\subsubsection{Background and role in xenobiotic metabolism}

The carbon monoxide-binding pigments of liver microsomes are hemoprotcins that have a characteristic absorption maximum near $450 \mathrm{~nm}$, and hence are called cytochromes P-450. The cytochromes P-450 enzymes comprise a family of hemoproteins with an identical prosthetic group (a thiolate-bound heme), but widely different apoprotein structures which are responsible for the different substrate specificities of reactions in which they are involved. Many families of cytochrome P-450 have been described including itn in mammals, two in insects, one in snail, one in plants and six in bacteria (Gonzalez, 1992). The ten mammalian cytochrome P-450 families can be functionally subdivided into two major classes; those involved in synthesis of steroids and bile acids (families $7,11,17,19,21$ and 27) and those that primarily metabolize xenobiotics (families 1, 2, 3 and 4) (Gonzalez, 1992). Xenobiotic-metabolizing cytochromes P-450) probably evolved from the cytochromes P-450 involved in steroid synthesis, and then began to oxidatively degrade dietary chemicals which were ensily eliminated from tnimals only after they were converted to more hydrophilic derivatives. The cytochromes P-450) are terminal oxidoreductases of the microsomal, nuclear membrane, mitochondrial and soluble monooxygenases. Microsomal cytochromes P.450 catalyze the metabolism of a wide variety of endogenous and exogenous compounds including drugs, steroids, prostaglandins, chemical carcinogens and other xenobiotics (Guengerich, 1991); Wislocki et al., 1980). They catalyze the incorporation of one atom of molecular oxygen into the substrate to give a product, while the other oxygen atom is reduced by two electrons to give water. Although these enzymes play an important role in the detoxification of many drugs, chemical carcinogens, and other toxic agents, they are also responsible for catalyzing the metabolic activation of some compounds to highly reactive intermediates which then react 
with critical cellular macromolecules and initiate toxic and carcinogenic events (Guengerich. 1991). The critical role these enzymes play, in the metabolic activation and detoxification of a wide variety of carcinogens and other toxic agents, makes them of particular importance in light of human exposure to these compounds in the environment. The microsomal cytochrome P-450-dependent mixed function oxidase enzyme system is composed of NADPH-cytochrome P-450 reductase, phospholipids, and cytochromes P450 , and for catalytic activity it exhibits an absolute requirement for $\mathrm{NADPH}$ and $\mathrm{O}_{2}$ (Guengerich, 1990 and 1991; Wislocki et al., 1980).

$\mathrm{RH}+\mathrm{O}_{2}+\mathrm{NADPH}+\mathrm{H}+\quad \begin{gathered}\text { cytochrome } \mathrm{P}-450 \\ \text { reductase }\end{gathered}$

The catalytic cycle for cytochrome P-450-catalyzed reactions is thought to consist of at least six discrete reactions (Hollenberg, 1992) (sec Figure 4). The first step involves binding of the substrate to the ferric form of the enzyme. The second step involves the transfer of one electron from the NADPH-cytorhrome P-450 reductase to the iron of the ferric cytochrome P.450 enzyme to give a ferrous enzyme-substrate complex. The reduced cytochrome P-450-substrate complex then binds $\mathrm{O}_{2}$ to form a ferrous enzyme- $\mathrm{O}_{2}$-substrate termary complex with the $\mathrm{O}_{2}$ bound to the iron. The addition of a second electron to this ternary complex by the reductase results in the formation of an iron peroxo species, best represented as $\mathrm{Fe}^{3}+\mathrm{O}_{2} 2$. The next step involves cleavage of the oxygen-oxygen bond. One of the oxygens is released with the uptake of two protons, resulting in the formation of water. The retained oxygen remains associated with the heme iron as an activated oxygen. The activated oxygen atom associated with the iron is then inserted into the substrate, resulting in a two-electron oxidation of the substrate to the alcohol (ic. hydroxylated 
substrate).

\subsubsection{Induction}

Some cytochrome P-450 isozymes are expressed constitutively, while others are expressed only in response to specific chemical stimuli (see Table 5). Examples of such chemical stimuli (inducers) are polycyclic aromatic compounds (c.g, 3tnethylcholanthrene), barbiturates (e.g. phenobarbital), and steroids (e.g. pregnenolone 16ex-carbonitrile) (more inducers and references to them are given in the Methods section 2.5.1). After induction, the activity of particular isoforms increases and the concentration of total cytochrome P-450 may increase. Elevated levels of cytochrome P-45() are the result of multiple mechanisms (Monostory et al., 1992). The major mechanism is increased transcription of cytochrome P-450 genes (higher levels of specilic mRNAs are detectable soon after treatment with some inducers of cytochrome P-450). A second mechanism is message stabilization and enhanced transport of mRNA from the nucleus to the cytoplasm so that mRNA levels at the site of protein synthesis are increased. A third mechanism is stabilization of cytochrome P-450 protein and/or mRNA (Monostory ct al., 1992). The fact that induction is produced by a large variety of xenobiotics and is mediated by multiple mechanisms suggests that the cytochrome $P-450$ system can provide increased protection for organisms in potentially hostile chemical environments. Since cytochrome P-450 induction is a characteristic response to chemicals of various tiving organisms ranging from microorganisms to man, it seems to be an adaptive mechanism for organisms to increase the chance for survival.

\subsubsection{Inhibition}

The catalytic cycle of cytochrome P-450 (see Figure 4) traverses three main steps that are particularly vulnerable to inhibition: 1) the binding of substrates, 2) the binding of 
molecular oxygen subsequent to the first electron transfer, and 3) the catalytic step in which the substrate is actually oxidized (Ortiz de Montellano and Reich, 1986). Cytochrome P450 inhibitors can be divided into three mechanistically differentiable categories: 1) agents that bind reversibly, 2) agents that form quasi-irreversible complexes with the heme iron atom, and 3) agents that bind irreversibly to the protein or the prosthetic heme group (Ortiz de Montellano and Reich, 1986). On the whole, inhibitors that interfere in the catalytic cycle prior to the actual oxidative event are reversible inhibitors, whereas agents that act during or subsequent to the oxygen transfer step are generally irreversible.

\subsection{3a Reversible Inhibitors:}

Inhibitors that compete reversibly with substrates for occupancy of the active site include substances that bind to its hydrophobic domain, that coordinate to the prosthetic heme iron atom, or that participate in specific hydrogen bonding or ionic interactions with specific active-site residues (Testa and Jenner, 1981) include the following:

i. Coordinators to ferric heme - e.g. cyanide (Kitada et al., 1977).

ii. Coordinators to ferrous heme - e.g. carbon monoxide (Hanson et al., 1976).

iii. Heme coordinators and lipophilic binders - e.g. metyrapone (Dominguez and Samuels, 1963).

\subsection{3b Catalysis-dependent inhibition:}

Several classes of inhibitors are known to be catalytically activated by the enzyme to transient species that irreversibly or quasi-irreversibly inhibit the enzyme. These mechanism-based (catalysis-dependent) inhibitors are potentially more enzyme-specific than reversible inhibitors because: 1) the inhibitor must first bind to the ferric enzyme, 2) the inhibitor must then be catalytically activated and therefore must be acceptable as a substrate, and finally, 3) the reactive species produced irreversibly alters the enzyme and renders it inactive (Ortiz de Montellano and Reich, 1986). Examples include compounds 
which undergo or cause:

i. Covalent binding to the protein - e.g. carbon disulfide (Bond and De Matteis, 1969).

ii. Quasi-irreversible coordination to the prosthetic heme - e.g. alkyl and aromatic amines (Testa and Jenner, 1981; Franklin, 1977).

iii. Covalent binding to the prosthetic heme - c.g. dihydropyridines and dihydroquinolines (heme alkylation) (Ortiz de Montellano and Correia, 1983; Gayarthri and Padmanaban, 1974).

iv. Heme degradation - e.g. carbon tetrachloride (Guzelian and Swisher, 1979). 


\section{Objectives of this work}

Recently, using a reconstituted microsomal lipid peroxidation system, we demonstrated that OTA induced lipid peroxidation by chelating $\mathrm{Fe}^{3+}$ ions, and that the resulting complex was more readily reducible by the flavoprotein NADPH cytochrome P450 reductase to the OTA-Fe2+ complex which, in the presence of oxygen, provided the active species that initiated lipid peroxidation (Omar et al., 1990). Cytochrome P-450 has been shown to play an important role in stimulating lipid peroxidation in ADP.Fe $3+$ system (Morehouse and Aust, 1988).

A number of studies have shown that OTA is metabolized by liver microsomes from several species (rat, pig and human) primarily to $4(\mathrm{R})-4-\mathrm{OH}-\mathrm{OTA}$ and to a smailer extent to the epimeric 4(S)-4-OH-OTA in the presence of NADPH (Stormer et al., 1981, 1983; Ueno, 1985; Creppy et al., 1991; Hietanen et al., 1986). OTA metabolism has also been shown to be induced by PB and/or 3MC in rats (Stormer and Pedersen, 1980; Ueno, 1985; Hietanen et al., 1986). In most of these studies, the effects of these cylochrome P450 inducers were not examined under the same experimental conditions. In addition, the effects of other important cytochrome P-450 inducers were never investigated.

\section{The objectives of my study were:}

i) To investigate the possible role of cytochrome P-450 in OTA-induced lipid peroxidation.

ii) To characterize which cytochrome P-450 isoforms are capable of metabolizing OTA through the use of a varicty of inducers, specific inhibitors and monoclonal antibodies.

iii) To examine the effect of cytochrome P-450 induction on OTA-induced toxicity in 
rats.

iv) To examine the effect of cytochrome P-450 induction on OTA absorption and distribution in rats. 
Figure 1 Structures of ochratoxin $\mathbf{A}$ and its metabolites. 


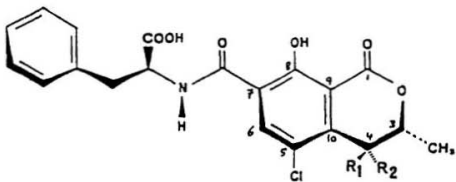

$$
\begin{array}{ll}
\text { Ochratoxin A (OTA) } & \mathrm{R}_{1}=\mathrm{R}_{2}=\mathrm{H} \\
4(\mathrm{R})-4 \text {-OH-OTA } & \mathrm{R}_{1}=\mathrm{OH}, \mathrm{R}_{2}=\mathrm{H} \\
4(\mathrm{~S})-4 \text {-OH-OTA } & \mathrm{R}_{1}=\mathrm{H}, \quad \mathrm{R}_{2}=\mathrm{OH}
\end{array}
$$


Table 1. Occurrence of OTA in food and animal feed of plant origin (adapted from WHO, 1990).

\begin{tabular}{|c|c|c|c|c|c|}
\hline Commodity & Country & $\begin{array}{l}\text { Samples } \\
\text { analyzed }\end{array}$ & $\%$ Cont. & $\begin{array}{l}\text { OTA range } \\
\text { (ug/kg) }\end{array}$ & Reference \\
\hline Wheat, hay & Canada & 95 & 7.4 & $30-6,000$ & Prior, 1976 \\
\hline $\begin{array}{l}\text { Wheat, oats, } \\
\text { barley, rye }\end{array}$ & Canada & 32 & 56.3 & $30-27,000$ & Scott et al., 1972 \\
\hline Mix. feed & Canada & 51 & 7.8 & $48-5,900$ & Abramson et al., 1983 \\
\hline Maize & Yugoslavia & 191 & 25.7 & $45-5,125$ & Balzer et al., 1977 \\
\hline Mix. feed & Australia & 25 & 4 & 70,000 & Connole et al., 1981 \\
\hline Flour & U.K. & 7 & 28.5 & $490-2,900$ & Osborne, 1980 \\
\hline Maize & U.K. & 29 & 37.9 & $50-500$ & $\begin{array}{l}\text { Ministry of } \\
\text { Agriculture, } 1980\end{array}$ \\
\hline Cornflour & U.K. & 13 & 30.8 & $50-500$ & " $"$ " \\
\hline Soya bean & U.K. & 25 & 36 & $50-500$ & $“$ \\
\hline $\begin{array}{l}\text { Cocoa beans } \\
\text { (raw) }\end{array}$ & U.K. & 56 & 17.9 & $100-500$ & $“$ \\
\hline Barley & Czechoslovakia & 48 & 2.1 & 3,800 & Vesela et al., 1978 \\
\hline Coffee beans & U.S.A & 267 & 7.1 & $20-360$ & Levi et al., 1974 \\
\hline wheat & U.S.A. & 577 & 3.8 & $5-115$ & Shottwell et al., 1976 \\
\hline Beans & Sweden & 71 & 8.5 & $10-442$ & $\begin{array}{l}\text { Akustrand and } \\
\text { Josefsson, } 1979\end{array}$ \\
\hline Maize & France & 924 & 3.9 & $15-200$ & Galtier et al., 1977b \\
\hline
\end{tabular}


Table 2. The incidence of OTA in meat and meat products (adapted from Marquardt et al., 1990).

\begin{tabular}{|c|c|c|c|c|c|c|c|}
\hline Product & Country & $\begin{array}{l}\text { Samples } \\
\text { analyzed }\end{array}$ & $\%$ Cont. & $\begin{array}{l}\text { OTA range } \\
\text { (ug/kg) }\end{array}$ & \multicolumn{3}{|c|}{ Reference } \\
\hline \multicolumn{8}{|l|}{ Kidney } \\
\hline Swine & Hurigary & 122 & 39 & $2-100$ & \multicolumn{3}{|c|}{ Jelinek et al., 1989} \\
\hline \multicolumn{8}{|l|}{ Meats } \\
\hline Ham & Yugoslavia & 206 & 29 & $40-70$ & “ & “ & “ \\
\hline Bacon & & & 19 & $37-200$ & “ & “ & “ \\
\hline Kulen & & & 13 & $10-460$ & $a$ & “ & “ \\
\hline Sausage & & & 12 & $10-920$ & “ & “ & $“$ \\
\hline \multicolumn{8}{|l|}{ Blood } \\
\hline Swine & Canada & 1200 & 0.5 & $100-229$ & \multirow{3}{*}{\multicolumn{3}{|c|}{ Marquardt et al., 1988}} \\
\hline & & & 4.1 & $20-229$ & & & \\
\hline & & & 72 & $5-20$ & & & \\
\hline
\end{tabular}


Figure 2 Factors influencing the occurrence of mycotoxins in human food and animal feed (redrawn from Smith and Moss, 1985). 


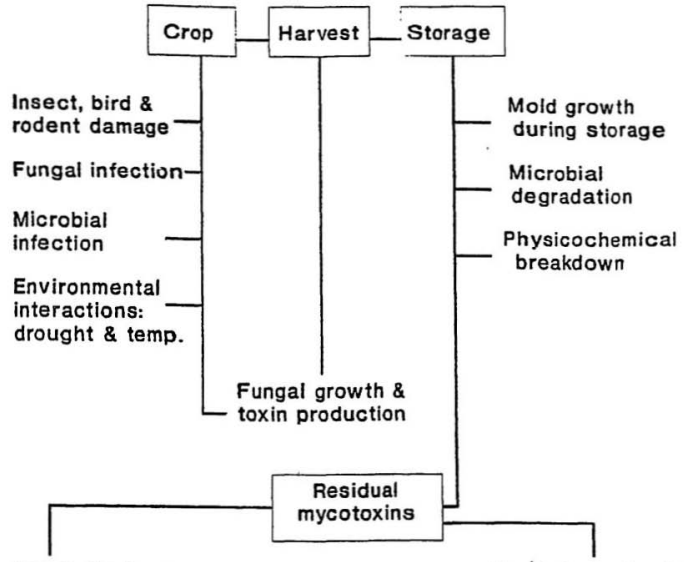

Foods for human

Feeds for animals consumption

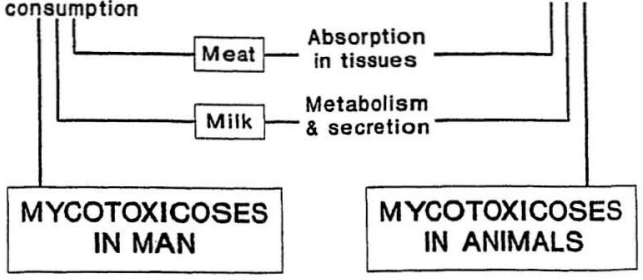


Table 3. OTA levels in humans (adapted from Kuiper-Goodman and Scott, 1989).

\begin{tabular}{llll}
\hline Sample $\quad$ Country $\quad$ Incidence & $\begin{array}{l}\text { OTA levels } \\
(\text { ug/kg or } / \mathbf{L})\end{array}$ & Reference \\
& &
\end{tabular}

Blood serum (from patients with urinary tract tumors/BEN)

$\begin{array}{lll}\text { Bulgaria } & 26 \% & \text { Up to } 35 \\ \text { (mean 20) } & \text { Petkova-Bocharova et al., } 1988\end{array}$

Blood serum (from non-endemic areas)

\begin{tabular}{|c|c|c|c|c|c|}
\hline \multirow{3}{*}{ Blood serum } & Bulgaria & 7.7 & mean 10 & “ $\quad \cdots$ & “ \\
\hline & Germany & $173 / 306$ & $0.1-14.4$ & \multicolumn{2}{|c|}{ Bauer and Gareis, 1987} \\
\hline & Germany & $3 / 46$ & $0.1-0.3$ & “ $"$ & “ \\
\hline Milk & Germany & $4 / 36$ & $0.02-0.03$ & $“$ & “ \\
\hline Blood serum & Poland & 9/216 & $1.3-4.8$ & \multicolumn{2}{|c|}{$\begin{array}{l}\text { Golinski and Grabarkiewicz-Szczesna, } \\
1985\end{array}$} \\
\hline \multicolumn{6}{|c|}{ Blood serum (from village with endemic nephropathy) } \\
\hline & Yugoslavia & $25 / 420$ & $1-40$ & \multicolumn{2}{|c|}{ Hult et al., 1982 a,b } \\
\hline \multicolumn{6}{|c|}{ Blood serum (from non-endemic village) } \\
\hline & Yugoslavia & $1 7 \longdiv { 2 1 9 }$ & $1-10$ & “ & “ \\
\hline
\end{tabular}


Table 4. LD $\mathbf{D}_{50}$ of OTA in different species.

\begin{tabular}{|c|c|c|}
\hline Species & $\begin{array}{l}\text { Route of } \\
\text { administration }\end{array}$ & $\mathrm{LD}_{50}(\mathrm{mg} / \mathbf{k G})^{*}$ \\
\hline Rat (male) & oral & 28.0 \\
\hline Rat (female) & oral & 21.4 \\
\hline Rat (male) & i.p. & 12.6 \\
\hline Rat (female) & i.p. & 14.3 \\
\hline Rat & i.v. & 12.7 \\
\hline Mouse & oral & 46.0 \\
\hline Mouse & i.p. & 22.0 \\
\hline Mouse & i.v. & 25.7 \\
\hline Dog & oral & 0.2 \\
\hline Guinea Pig (m) & oral & 9.1 \\
\hline Guinea Pig (f) & oral & 8.1 \\
\hline Chicken & oral & 3.3 \\
\hline Turkey & oral & 5.9 \\
\hline RainbowTrout & i.p. & 4.7 \\
\hline
\end{tabular}

* Adapted from WHO, 1990 and MSDS, Sigma Chemical Co., 1989. 
Figure 3 Simplified reactions of the process of lipid peroxidation (Gutteridge, 1988). 


\section{Lipid peroxidation}
(1) $\mathrm{LH}+\mathrm{R}^{\circ} \longrightarrow \mathrm{L}^{\circ}+\mathrm{RH}$
Initiation
(2) $\mathrm{L}^{\circ}+\mathrm{O}_{2} \longrightarrow \mathrm{LOO}^{\circ}$ $\mathrm{LH}+\mathrm{LOO}^{\circ} \longrightarrow \mathrm{LOOH}+\mathrm{L}^{\circ}$ Propagatiol
(3) $L^{\dot{L}}+\dot{L}^{\circ}$ $\mathrm{LOO}^{\circ}+\mathrm{LOO}^{\circ} \longrightarrow \mathrm{LOOL}+\mathrm{O}_{2}$ $\mathrm{LOO}^{\circ}+\mathrm{L}^{\circ} \longrightarrow \mathrm{LOOL}$
Terminatior

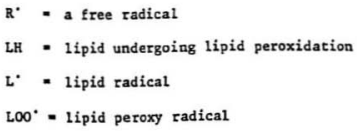


Figure 4 The catalytic cycle of cytochrome P-450-catalyzed reactions and OTA hydroxylation. 


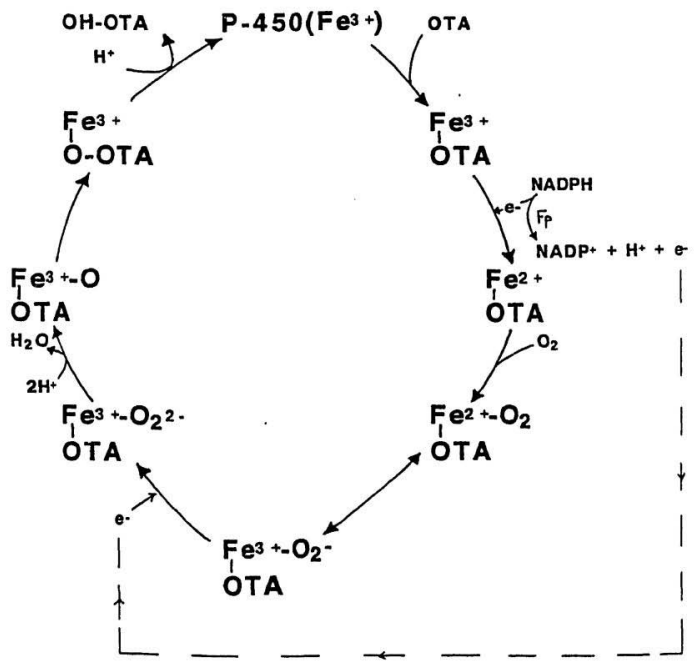


Table 5. Regulation of P-450 gene expression (Adapted from Soucek and Gut., 1992).

CYP Regulation of gene expression

IA1 Inducible (all tissues) - 3MC, ISF, BNF: transcriptional activation + mRNA stabilization.

IA2 Constitutive (liver): transcriptional activation + mRNA stabilization. Inducible (all tissues) - 3MC, ISF, BNF: transcriptional activation + mRNA stabilization.

IIB1 Constitutive (lung, testis) - age, strain, colony, growth hormone: transcriptional activation.

Inducible (all tissues) - PB, starvation: transcriptional activation + mRNA stabilization.

IIE1 Constitutive (liver, kidney) - age, growth hormone: transcriptional activation; diabetes, starvation: transcriptional activation + mRNA stabilization.

Inducible (liver, lung) - INH, EtOH: protein stabilization.

IIIA1 Inducible - PCN, DEX, hypertension: transcriptional activation + mRNA stabilization.

III2 Constitutive (liver, intestine) - age, sex (m), growth hormone, diabetes, starvation: transcriptional activation.

Inducible - PB: activation.

IVAl Constitutive (liver, kidney): transcriptional activation. Inducible - Clofibrate, starvation, transcriptional activation. 


\section{CHAPTER 2}

\section{MATERIALS AND METHOHS}

2.1 Chemicals - Ascorbate, bathophenanthroline disulfonic acid, butylated hydroxyanisole, catalase, clofibrate, $\mathrm{FeCl}_{3}, \mathrm{FeSO}_{4}, \mathrm{H}_{2} \mathrm{O}_{2}$, isocitric acid, isocitric dehydrogenase, isoniazid, isosafrole, lipoxygenase, mannitol, 3-methylcolanthrene, metyrapone, NADPH, $\alpha$-naphthoflavone, ochratoxin A, phenobarbital, pregnenolone-16 $\alpha-$ carbonitrile, superoxide dismutase and 2-thiobarbituric acid were purchased from Sigma Chemical Co. (St. Louis, MO). [3 $\left.{ }^{3}\right]$ Ochratoxin A was obtained from Amershan, U.K. and further purified by thin layer chromatography (TLC), 7-Ethoxyresorufin, 7pentoxyresorufin and resorufin were purchased from Pierce (Rockford, IL). Co-protoporphyrin IX was obtained from Porphyrin Products (Logan, UT, USA). Sephadex G-50 was obtained from Pharmacia (Toronto, Canada). All other chemicals were of the highest grade commercially available.

2.2 TLC purification of [3H]OTA - Two $\mathrm{mL}$ of $[3 \mathrm{H}] \mathrm{Och}$ ratoxin $\mathrm{A}$ was uscd, the solvent was evaporated under a stream of nitrogen and the residue was redissolved in methanol. The whole sample was applied as a streak along the lower part (the origin) of a thin layer chromatography (TLC) plate (K5F silica gel, layer thickness $250 \mu$, Whatman). Standard OTA was applied as a spot. The chromatogram was developed with benzene : acetic acid ( $4: 1, v / v)$, and after evaporation of solvent examined under U.V. light. The silica gel with the band corresponding to OTA was scraped off (removed), and OTA cluted with methanol. The solution contained $83 \%$ of the total radioactivity. The latter OTA from the first chromatography was further purified on a second TLC plate following the same steps described above and the resulting OTA solution from the second chromatography contained $92 \%$ of the total radioactivity. 
2.3 Preparation of ochratoxin A - OTA was purchased from Sigma chemical Co., but was also produced in the lab according to the procedures described in my Master's thesis (Omar, 1990). The UV and fluorescence spectra of OTA are given in Figures 5 and 6. The purity of the prepared toxin was checked by TLC (Table 6) and HPLC (Figure 7). The identity of the toxin was checked by NMR (Figure 8). OTA concentration was calculated using the absorption coefficient at $332 \mathrm{~nm}$ of $6330 \mathrm{~cm}^{-1} \mathrm{M}^{-1}$ (Pohland et al., 1982).

2.4 Animals - Male Sprague-Dawley rats (200-250 g), obtained from Canadian Breeding Farms, Halifax, Nova Scotia, were used in all experiments. Animals were maintained on a $12 \mathrm{hr}$ light and dark cycle and had free access to standard laboratory chow and water.

\subsection{Treatments of rats for in vitro experiments}

2.5.1 Treatments with cytochrome P.450 inducers - The various treatments (n $=4 /$ treatment) are listed in Table 7 . Isoniazid was given as a $0.1 \%$ solution in drinking water for 10 days and rats were sacrificed on day 11 (Ryan et al., 1985). PCN $(100 \mathrm{mg} / \mathrm{kg}$ body weight in $1 \mathrm{~mL}$ of $1 \%$ Tween 80 ) was administered by gastric intubation once daily for 4 days and rats were sacrificed $24 \mathrm{hr}$ after the last dose (Graves et al., 1987). PB was given as a $0.1 \%$ solution in drinking water for 5 days and rats were sacrificed at the end of that time period (Graves et al., 1987). 3MC (20 mg/kg body weight in corn oil) was administered intraperitoneally once each day for 3 successive days, and rats were sacrificed $24 \mathrm{hr}$ after the last dose (Guengerich et al., 19820). Clofibrate was administered by gastric intubation (200 mg/kg in $1 \mathrm{~mL}$ sucrose syrup containing $1 \%$ gum arabic) once each day for 5 consecutive days, and rats were sacrificed $24 \mathrm{hr}$ after the last dose (Fournel et al., 1987). Isosafrole (120 mg/kg body weight in corn oil) was given intraperitoneally once each day 
for 3 successive days, and rats were sacrificed 24 hr after the last dose (Fisher et al., 1981).

\subsubsection{Treatments with Cobalt protoporphyrin IX for microsomal} preparations - Rats received 2 doses each of $50 \mu \mathrm{mol} / \mathrm{kg}$ body weight of cobalt protoporphyrin IX (Co-heme) 9 days and 2 days prior to killing, while control rats received the vehicle saline at the same time as the treated rats (Drummond and Kappas, 1982). Coheme $(24.8 \mathrm{mg}$ ) was dissolved in $0.4 \mathrm{~mL}$ of $0.1 \mathrm{M} \mathrm{NaOH}$, the $\mathrm{pH}$ was adjusted to 7.4 with $\mathrm{HCl}$ and the solution was made up to $4 \mathrm{~mL}$ with nomal saline (final concentration of Co-heme, $10 \mathrm{mM}$ ). The freshly prepared Co-heme was administered subcutaneously to rats at a dose of $0.5 \mathrm{~mL} / \mathrm{I00} \mathrm{gm}$ body weight. After sacrifice, liver microsomes showed $20 \%$ of cytochrome P-450 content of controls.

\subsection{Treatments of rats for In vivo studies .}

2.6.1 Inducing cytochrome P-450 - Four rats were used per treatment group. Control rats had free access to drinking water and standard lab chow for 5 days, whereas PB treated rats were given $0.1 \% \mathrm{~PB}$ in drinking water for five days (as the control rats, $\mathrm{PB}$ treated rats also had free access to the drinking water with PB and standard lab chow) (Graves et al., 1987). Animals were maintained on a $12 \mathrm{hr}$ light and dark cycle. On the fifth day, all animals ( 8 rats) were placed individually in metabolic cages and $24 \mathrm{hr}$ urines were collected (for a base line). On the sixth day, all rats received OTA by gastric intubation ( $0.5 \mathrm{mg} / \mathrm{kg}$ body weight in $50 \mathrm{mM}$ sodium bicarbonate, about $0.5 \mathrm{~mL}$ ); this treatment of OTA was given for five successive days. Twenty four hour urines were collected every day until one day after the last OTA treatment. After sacrifice, liver microsomes from PB treated rats showed a 2.5 fold increase in cytochrome P-450 contents as compared to control microsomes. 
2.6.2 [3H]OTA experiments - Rats were divided into two groups $(n=4 /$ treatment), one group received drinking water whereas the other received water containing $0.1 \% \mathrm{~PB}$ for five days before OTA treatment. All animals had free access to food (standard lab chow). After the five days, both groups were treated with [3H]OTA $(288 \mu \mathrm{g} / \mathrm{kg}$ body weight in $50 \mathrm{mM} \mathrm{NaHCO}$ once by gastric intubation). Rats were kept in metabolic cages for $6 \mathrm{hr}$ to collect urine.

2.7 Preparation of liver microsomes - Liver microsomes werc isolated by differential centrifugation of liver homogenates as described earlier (Rahimtula et al., 1979). Liver from each rat was excised and placed in ice-cold $0.1 \mathrm{M}$ potassium phosphate buffer, $\mathrm{pH}$ 7.4. The liver was chopped into pieces, homogenized in 3 parts (by volume) of the above buffer to one part liver (wet weight) using a polytron homogenizer (Brinkman Instruments), and the homogenate was centrifuged at $10,000 \times \mathrm{g}$ for $10 \mathrm{~min}$. Following filtration of the resulting supernatant through cheesecloth, microsomes were isolated from the $10,000 \times \mathrm{g}$ supernatant by ultracentrifugation at $110,000 \times \mathrm{g}$ for $60 \mathrm{~min}$. The microsomal pellet was suspended in $0.1 \mathrm{M}$ potassium phosphate buffer, $\mathrm{pH} 7.4$ and again centrifuged at $110,000 \times \mathrm{g}$ for $60 \mathrm{~min}$. The final microsomal pellet was suspended in 0.1 $\mathrm{M}$ potassium phosphate buffer, $\mathrm{pH} 7.4$ and stored at $-70^{\circ} \mathrm{C}$ until used. Protein was measured by the Lowry method (Lowry et al., 1951). Cytochrome P-450 levels were measured as described by Omura and Sato (1964) (see below, section 2.9). NADPHcytochrome P-450 reductase (Fp) was assayed as described by Lake (1987) using cytochrome $c$ as the electron acceptor (explained below, section 2.11).

2.8 Cytochrome P-450 purification - Cytochrome P-450 was purified from liver microsomes isolated from PB-pretreated rats as described by Guengerich (1982). All steps 
were carried out at $4^{\circ} \mathrm{C}$. Microsomes were suspended to $2 \mathrm{mg}$ protein/ $/ \mathrm{mL}$ in $0.1 \mathrm{M}$ potassium phosphate buffer ( $\mathrm{pH} 7.25$ ) containing $20 \%$ glycerol, $1 \mathrm{mM}$ EDTA, and $20 \mu \mathrm{M}$ BHT. Sodium cholate (recrystallized from $50 \%$ aqueous ethanol) was added dropwise (from a separatory funnel) to the stirring suspension over $20 \mathrm{~min}$ to give a final concentration of $0.6 \%$ (weigh/volume, w/v). After stirring for an additional $30 \mathrm{~min}$, the clarified solution was centrifuged at $100,000 \times \mathrm{g}$ for $1 \mathrm{hr}$. An amount of the supernatant equivalent to 2,000 nmoles of cytochrome P-450 was applied at a flow rate of $1 \mathrm{~mL} / \mathrm{min}$ to an w-amino-octyl agarose column $(2.5 \times 50 \mathrm{~cm})$ previously equilibrated with 30$) \mathrm{mL}$ of $0.1 \mathrm{M}$ potassium phosphate buffer ( $\mathrm{pH} 7.25$ ) containing $1 \mathrm{mM}$ EDTA, 20\% glycerol, and $0.6 \%(\mathrm{w} / \mathrm{v})$ sodium cholate. The cytochrome $\mathrm{P}-450$, a reddish brown protein, was bound to the top one-third of the column. The column was washed with $800 \mathrm{~mL}$ of $0.1 \mathrm{M}$ potassium phosphate buffer ( $\mathrm{pH} 7.25$ ) containing 1 niM EDTA, 20\% glycerol (volume/volume, $v / v$ ) and $0.42 \%(w / v)$ sodium cholate. Cytochrome $\mathrm{P}-450$ was eluted using about $1,500 \mathrm{~mL}$ of $0.1 \mathrm{M}$ potassium phosphate buffer containing $1 \mathrm{mM}$ EDTA, 20\% glycerol, $0.33 \%$ (W/v) sodium cholate and $0.06 \%$ (w/v) Renex 690 (ICI Americas Inc., WA, U.S.A.). The eluted fractions were monitored for cytochrome $\mathrm{P}-450$ by measuring the absorption at $417 \mathrm{~nm}\left(\mathrm{~A}_{417}\right)$. The $\mathrm{A}_{417}$ peak fractions were pooled and concentrated to about $50 \mathrm{~mL}$ using an Amicon ultrafiltration apparatus and a PM-30 membranc. The concentrated solution was dialyzed against $1 \mathrm{~L}$ of a $20 \%$ glycerol- $0.1 \mathrm{mM}$ EDTA solution (about $3 \mathrm{hr}$ ) and then versus $1 \mathrm{~L}$ of $10 \mathrm{mM}$ potassium phosphate buffer ( $\mathrm{pH} 7.7$ ) containing $0.1 \mathrm{mM}$ EDTA, $20 \%$ glycerol, $0.1 \%(w / v)$ Lubrol PX and $0.2 \%(w / v)$ sodium cholate (not recrystallized) (about $3 \mathrm{hr}$ ).

The cytochrome P-450 was further purified by DEAE cellulose chromatography at room temperature (about $22^{\circ} \mathrm{C}$ ). The dialyzed cytochrome $\mathrm{P}-450$ solution was applied to a $2.5 \times 50 \mathrm{~cm}$ column of Pharmacia DEAE-Sephacel previously equilibrated with $1 \mathrm{~L} \mathrm{of} 10$ 
$\mathrm{mM}$ potassium phosphate buffer ( $\mathrm{pH} 7.7$ ) containing $0.1 \mathrm{mM}$ EDTA, $20 \%$ glycerol, $0.1 \%$ $(w / v)$ Lubrol PX and $0.2 \%(w / v)$ sodium cholate (not recrystallized). The column was washed with $700 \mathrm{~mL}$ of the same buffer in which the concentration of $\mathrm{NaCl}$ was increased linearly to $0.25 \mathrm{M}$. The last major $\mathrm{A}_{417}$ peak contains the bulk of the cytochrome P-450. The peak fractions were pooled and concentrated to about $20 \mathrm{~mL}$ with an Amicon ultrafiltration apparatus using a PM-30 membrane. The concentrated solution was stirred with Bio-Beads SM-2 (Bio-Rad Labs; $0.2 \mathrm{gm} / \mathrm{mg}$ protein) for $3 \mathrm{hr}$ to remove excess detergent and then filtered through glass wool. Finally, the enzyme preparation (cytochrome P-450 IIB1) was dialyzed overnight against 50 volumes of $10 \mathrm{mM}$ Trisacetate buffer ( $\mathrm{pH} 7.4$ ) containing $0.1 \mathrm{mM}$ EDTA and $20 \%$ glycerol. The dialyzed enzyme was stored in aliquots $\left(0.25 \mathrm{~mL}\right.$ each) at $-80^{\circ} \mathrm{C}$. SDS-polyacrylamide gel electrophoresis revealed the presence of a single major protein band with a very minor band undemeath.

2.9 Cytochrome P-450 assay - Cytochrome P-450 concentration was measured spectrophotometrically as described by Omura and Sato (1964) using an extinction coefficient of $91 \mathrm{~cm}^{-1} \mathrm{mM}^{-1}$. Briefly, $6 \mathrm{mg}$ microsomal protein or $0.6 \mathrm{mg}$ protein of the cytochrome $\mathrm{P}-450$ preparation was suspended in $6 \mathrm{~mL}$ of $0.1 \mathrm{M}$ potassium phosphate buffer (pH 7.4) and a few small crystals of sodium dithionite were added to it. After mixing, the contents were divided between 2 matched cuvettes ( $3 \mathrm{~mL}$ in each) and scanned between $500-400 \mathrm{~nm}$ (scan speed $120 \mathrm{~nm} / \mathrm{min}$, chart speed $30 \mathrm{~mm} / \mathrm{min}$ ) to obtain a baseline. The sample cuvette was bubbled for about $\mathbf{4 0} \mathrm{sec}$ to $1 \mathrm{~min}$ with $\mathrm{CO}$ gas and then re-scanned to obtain the characteristic peak for the cytochrome P-450 at $450 \mathrm{~nm}$. My cytochrome $\mathrm{P}-450$ preparation contained $8.25 \mathrm{nmol} / \mathrm{mg}$ protein which was lower than expected, but it showed a complete absence of NADPH cytochrome P-450 reductase activity (Fp). Morehouse and Aust, 1988 satisfactorily used a cytochrome P-450 
preparation which contained $10 \mathrm{nmol} / \mathrm{mg}$ protein. Also, Laethem et al. (1992) purified cytochrome P-450 with specific activity of $8.4 \mathrm{nmol} / \mathrm{mg}$ protein. Usually, some of the enzyme activity is lost during the purification and also some heme-degradation occurs with the use of detergents. Attempts to restore cytochrome $\mathrm{P}-450$ by addition of heme has not met with success so far.

\subsection{NADPH-cytochrome P-450 reductase purification - The flavoprotein} NADPH-cytochrome $\mathrm{P}-450$ reductase (Fp) was purified from liver microsomes isolated from PB-pretreated rats essentially as described by Ardies et al. (1987). The following procedures were all performed at $4{ }^{\circ} \mathrm{C}$. Microsomes $(50 \mathrm{mg}$ protein $/ \mathrm{mL})$ were diluted to 10 $\mathrm{mg}$ protein/mL with $100 \mathrm{mM}$ Tris-HCl buffer (pH 7.7) containing $1.0 \mathrm{mM}$ EDTA, $1.0 \mathrm{mM}$ dithiothreitol (DTT), $20 \mu \mathrm{M}$ BHT, $5 \mu \mathrm{M}$ flavin mononuclcotide (FMN), and $30 \%$ glycerol (buffer A). CHAPS (3-[(cholamidopropyl)-dimethylammoniol-1-propsnesulfonate) was prepared as a $20 \%(\mathrm{w} / \mathrm{v})$ solution in water, and neutralized to $\mathrm{pH} 7.4$ with dilute $\mathrm{KOH}$. Immediately prior to use, CHAPS was diluted 1:1 (v/v) with buffer $A$. The latter solution of $10 \%$ CHAPS (in buffer $A$ ) was added to the microsomes slowly dropwise with stirring to a final concentration of $1 \%$ and the mixture was stirred for $30 \mathrm{~min}$. Then, a $1.5 \%$ solution of protamine sulfate was added dropwise to a final concentration of $0.07 \%(w / v)$. After stirring for an additional $20 \mathrm{~min}$, the mixture was centrifuged at $110,00(1) \times \mathrm{g}$ for 60 min. The supernatant was removed, and the resulting gray-colored, tightly packed pellet was resuspended with the aid of a Teflon/glass homogenizer at a protein concentration of $50 \mathrm{mg} / \mathrm{mL}$ in buffer A; protein was measured by the Lowry method (Lowry et al., 1951). A $10 \%(w / v)$ solution of sodium cholate in water was then added dropwise with stirring to a final detergent : protein ratio of $3 \mathrm{mg} / \mathrm{mg}$. Ten minutes later, a $20 \%(\mathrm{v} / \mathrm{v})$ solution of Lubrol PX (Sigma Chemical Co., MO, USA) in water was added dropwise to a final concentration of $0.5 \%(\mathrm{w} / \mathrm{v})$ and the mixture was stirred for an additional $30 \mathrm{~min}$. The 
detergent-treated fraction was centrifuged at $110,000 \times \mathrm{g}$ for $60 \mathrm{~min}$, and the resulting supernatant was applied directly to a $2^{\prime}, 5^{\prime} \cdot$ ADP agarose column $(2.5 \times 4.0 \mathrm{~cm})$ at a flow rate of $1 \mathrm{~mL} / \mathrm{min}$. The affinity column had been equilibrated previously with $100 \mathrm{mM}$ phosphate buffer (pH 7.7) containing $0.4 \%$ cholate, 0.1 mM EDTA, $0.1 \mathrm{mM}$ DTT, $20 \mu \mathrm{M}$ BHT, $5 \mu \mathrm{M}$ FMN, and $20 \%$ glycerol. Once loaded, the column was washed with 15 column volumes of equilibration buffer, 12 column volumes of $100 \mathrm{mM}$ phosphate buffer (pH 7.7) containing $1.0 \%(\mathrm{v} / \mathrm{v})$ CHAPS, $0.5 \%(\mathrm{v} / \mathrm{v})$ Lubrol PX, $0.1 \mathrm{mM}$ EDTA, $0.1 \mathrm{mM}$ DTT, $20 \mu \mathrm{M}$ BHT, $5 \mu \mathrm{M}$ FMN, and $20 \%(v / v)$ glycerol, and again with 15 column volumes of equilibration buffer. All washes were performed at a flow rate of a $1 \mathrm{~mL} / \mathrm{min}$. Fp was then eluted from the affinity resin with a small volume (about $25 \mathrm{~mL}$ ) of equilibration buffer to which $10 \mathrm{mM} \mathrm{NADP}+$ had been added (flow rate $=0.5 \mathrm{~mL} / \mathrm{min}$ ). The peak Fp-containing fractions (detected by measuring Fp in fractions as described below, section 2.11) were pooled and dialyzed twice against $2 \mathrm{~L}$ of $50 \mathrm{mM}$ phosphate buffer (pH 7.4) containing $0.1 \mathrm{mM}$ DTT and $20 \%$ (v/v) glycerol for a total of $28 \mathrm{hr}$. The purified $\mathrm{Fp}$ was then stored in small aliquots $(0.5 \mathrm{~mL}$ each $)$ at $-800 \mathrm{C}$. The purified $\mathrm{Fp}$ showed a single band on sodium dodecyl sulfate-polyacrylamide gel electrophoresis.

\subsection{NADPH-cytochrome P-450 reductase assay - The enzyme was assayed as} described by Lake (1987) using cytochrome $c$ as the electron acceptor. Briefly, $1 \mathrm{~mL}$ of $0.125 \mathrm{mM}$ cytochrome $c$ solution in $0.1 \mathrm{M}$ phosphate buffer, $\mathrm{pH} 7.0$, and $0.2 \mathrm{~mL}$ of 15 $\mathrm{mM} \mathrm{KCN}$ in water were pipetted into each of two matched $3 \mathrm{~mL}$ spectrophotometer cuvettes. Ten microliters of $\mathrm{Fp}$ was pipetted into each cuvette and phosphate buffer was added to the test and reference cuvette contents to bring the volumes up to 2.4 and $2.5 \mathrm{~mL}$, respectively. After mixing, the cuvettes were placed in the spectrophotometer (thermostatted to $22^{\circ} \mathrm{C}$ ), and $3 \mathrm{~min}$ later the reaction was initiated by adding $0.1 \mathrm{~mL}$ of 10 
mM NADPH to the test cuvette only. The contents were again mixed and the increase in absorbance with time was recorded at $550 \mathrm{~nm}$. Using an extinction coefficient for the reduced cytochrome $c$ at $550 \mathrm{~nm}$ of $0.021 \mathrm{~cm}^{-1} \mu \mathrm{M}-1$, the specific activity of the flavoprotein was calculated to be 18,000 units (or nmol)/min/mg protein (Lake, 1987). One unit of enzyme activity is defined as that amount which catalyzes the reduction of 1 nmol cytochrome $c / \mathrm{min}$.

2.12 Fortification of microsomes from Co-heme treated rats with $\mathrm{Fp}$ - Coheme drastically depleted cytochrome P-450 ( $>80 \%$ depletion of cytochrome P-450) content compared to controls). NADPH-cytochrome P-450 reductase (Fp) was also depleted (about 75\% depletion). To avoid the possible effect of reduced Fp, Co-heme microsomes were fortified by incubating them with purified $\mathrm{Fp}$. Microsomes $(2 \mathrm{mg}$ protein) were incubated with Fp $(640 \mathrm{nmol})$ in a little microfuge tube (about $150 \mu \mathrm{L}$ capacity) for $1 \mathrm{hr}$ at $22^{\circ} \mathrm{C}$ and then centrifuged at $110,000 \mathrm{xg}$ for $5 \mathrm{~min}$ to collect microsomes. The microsomes were resuspended in buffer and assayed for Fp content as described above. Forified microsomes were found to have a Fp content of $70 \mathrm{nmol} / \mathrm{mg}$ protein as compared to $20 \mathrm{nmol} / \mathrm{mg}$ protein in non-fortified microsomes.

\subsection{Preparation of phospholipid vesicles - Total lipid was extracted from} untreated rat liver microsomes by the method of Folch et al, (1956) with care being taken to flush all solvents with nitrogen and to perform all operations under nitrogen at $0-4^{\circ} \mathrm{C}$ to minimize auto-oxidation of polyunsaturated lipids. The extracted lipid in chloroform : methanol $(2: 1)$ was stored in aliquots under nitrogen at $-80^{\circ} \mathrm{C}$. Total lipid phosphorus was determined as described by Barlett (1959). Phospholipid vesicles were preparcd fresh daily by sonication of the extracted lipid under anaerobic conditions as described by 
Pederson et al. (1973). Briefly, an aliquot of the phospholipid solution was evaporated to dryness in a plastic tube under nitrogen, and nitrogen-saturated Tris- $\mathrm{HCl}$ buffer $(0.25 \mathrm{M}$, $\mathrm{pH} 6.8$ ) was added to give a final lipid phosphorus concentration of $10 \mu \mathrm{mol} / \mathrm{mL}$. The tube was flushed with nitrogen, capped, and placed in a glass beaker filled with a mixture of ice and water. Phospholipid vesicles were obtained by placing the probe of a Branson sonifier (model W185) in the beaker and applying a power of $50 \mathrm{~W}$ for $5 \mathrm{~min}$.

\subsection{Lipid peroxidation assays (incubation conditions) -}

2.14.1 Reconstituted system incubations - Unless otherwise specified, incubations were carried out in duplicate at $37^{\circ} \mathrm{C}$ in $0.25 \mathrm{M} \mathrm{Tris}-\mathrm{HCl}$ buffer $/ 0.25 \mathrm{M} \mathrm{NaCl}$ (pH 6.8) and contained per mL: phospholipid vesicles ( $1 \mu \mathrm{mol}$ P), $3.2 \mathrm{U} \mathrm{Fp}, 110 \mathrm{nmol}$ $\mathrm{Fe}^{3}, 500 \mathrm{nmol}$ OTA, and $200 \mathrm{nmol}$ NADPH. Cytochrome P-450 IIB1 or EDTA, when included, were added at the indicated concentrations (see Table 8).

2.14.2 Microsomal incubations - Incubations were carried out at $37^{\circ} \mathrm{C}$ in $0.1 \mathrm{M}$ potassium phosphate buffer ( $\mathrm{pH} 7.4$ or different $\mathrm{pHs}$ as indicated in the figure legends) and contained per mL: 2 mg microsomal protein (from either control animals, those induced, those treated with $\mathrm{Co}$-heme, or those treated with $\mathrm{Co}_{0}$-heme and microsomes fortified with Fp), 125 nmol OTA and $1 \mathrm{mM} \mathrm{NADPH}$.

Lipid peroxidation was estimated by measuring MDA levels (Rahimtula et al., 1988). For this purpose, $0.5 \mathrm{~mL}$ of $30 \% \mathrm{TCA}$ and $50 \mu \mathrm{L}$ of BHT ( $2 \%$ in ethanol) were added to $0.5 \mathrm{~mL}$ of each incubation. Finally, $0.5 \mathrm{~mL}$ of $50 \mathrm{mM}$ TBA was added and the mixtures were placed in a boiling water bath for $15 \mathrm{~min}$. After centrifugation for $5 \mathrm{~min}$ at $2000 \mathrm{rpm}$ (bench top centrifuge), the absorbance of the MDA-TBA complex in the supernatant was read at $535 \mathrm{~nm}\left(E_{535}=156 \mathrm{mM}^{-1} \mathrm{~cm}^{-1}\right)$ (Jordan and Schenkman, 1982). 


\subsection{Metabolic studies}

2.15.1 Metabolism of OTA In vitro - Unless otherwise indicated, incubations were carried out in duplicate at $370 \mathrm{C}$ for $30 \mathrm{~min}$ in $0.1 \mathrm{M}$ potassium phosphate buffer $(\mathrm{pH}$ 7.4) and contained in a total volume of $1 \mathrm{~mL}: 2 \mathrm{mg}$ microsomal protein, $125 \mathrm{nmol}$ OTA and a NADPH-regenerating system (consisting of $0.4 \mu \mathrm{mol} \mathrm{NADP}+, 5 \mu \mathrm{mol} \mathrm{MgCl}_{2}, 5 \mu \mathrm{mol}$ DL-isocitrate and 0.65 units of isocitric dehydrogenase). Catalase ( 800 units), SOD (35 units), mannitol (11 mM), BHA $(10 \mu \mathrm{M})$, DPPD $(10 \mu \mathrm{M})$, Desferal $(50 \mu \mathrm{M})$ or BPS (100) $\mu \mathrm{M})$, when included, were added prior to initiating the reaction with NADPH. At the end of $30 \mathrm{~min}$, a $0.5 \mathrm{~mL}$ aliquot from each incubation was withdrawn for measurement of OTA metabolites and the remaining $0.5 \mathrm{~mL}$ was used to measure lipid peroxidation.

Time-course studies were carried out in an identical manner except that the incubation volume was $10 \mathrm{~mL}$.

Incubations with hemoproteins and hydroperoxides were carried out in duplicate in $0.5 \mathrm{~nL}$ of $0.1 \mathrm{M}$ potassium phosphate buffer ( $\mathrm{pH} 7.4)$ and contained (i) OTA (62.5 nmol), (ii) hemoprotein - microsomes from PCN treated rats (1 mg protein; $1.2 \mathrm{nmol}$ cylochrome P-450) or $\mathrm{Hb}(640 \mu \mathrm{g} ; 10 \mathrm{nmol})$ or hematin $(2.5 \mathrm{nmol})$ or HRP $(400 \mu \mathrm{g} ; 10 \mathrm{nmol})$ and (iii) hydroperoxide - $\mathrm{CHP}(0.5 \mathrm{mM})$ or $\mathrm{H}_{2} \mathrm{O}_{2}(5 \mathrm{mM})$ or LAHP $(65 \mu \mathrm{M})$. Incubations were terminated after $30 \mathrm{~min}$ and analyzed for OTA metabolites as described below. LAIIP was prepared from linoleic acid and lipoxygenase as described earlier (O'Brien, 1969).

\subsubsection{Effect of monoclonal antibodies on OTA metabolism in vitro .} Monoclonal antibodies (MAbs) were obtained from Dr. H. Gelboin, U.S. National Cancer Institute, Lab of Molecular Carcinogenesis, Bethesda, MD. Clones 1-7-1 (against cytochrome P-450 IA1/IA2) and 2-66-3 (against cytochrome P-450 IIB 1/IIB2) were used in my studies. Rabbit IgG was used to determine any non-specific reaction. Microsomes 
were preincubated with MAbs in buffer at room temperature 30 min (Nakajima et al., 1991) prior to initiating the OTA metabolism assay at $370^{\circ} \mathrm{C}$ by addition of a NADPH-regenerating system and substrate (OTA).

2.15.3 Analysis of OTA metabolites formed in in vitro studies - Aliquots (0.5 $\mathrm{mL}$ ) were removed from incubations, and the reaction was terminated by the addition of 1 $\mathrm{M} \mathrm{HCl}(0.1 \mathrm{~mL})$ and saturated $\mathrm{NaCl}(0.5 \mathrm{~mL})$. The mixtures were extracted with chloroform $(2 \times 2 \mathrm{~mL})$, and the two chloroform extracts from each incubation were combined and dried under nitrogen. The residues were dissolved in $500 \mu \mathrm{L}$ of methanol and $50 \mu \mathrm{L}$ of each sample was analyzed by HPLC (Perkin Elmer, Series 4) on a Partisil 10 ODS-2 column $(0.45 \mathrm{~cm} \times 25 \mathrm{~cm})$ using a solvent system consisting of (i) a mixture of acetonitrile : methanol $(1: 1, \mathrm{v} / \mathrm{v}) 60 \%$ and (ii) $5 \mathrm{mM}$ sodium acetate : acetic acid $(500: 14$, $\mathrm{v} / \mathrm{v}) 40 \%$. The flow rate was $1.5 \mathrm{~mL} / \mathrm{min}$. Ochratoxin $\mathrm{A}$ and its metabolites were detected fluorimetrically using light at $340 \mathrm{~nm}$ for excitation and measuring the emission at $465 \mathrm{~nm}$. 4(S)-4-OH-OTA, 4(R)-4OH-OTA and OTA eluted at 4.2, 5.1 and $10.1 \mathrm{~min}$ respectively. Metabolites were identified and quantitated using standards generously provided by $\mathrm{Dr}$. M. Castegnaro, IARC, Lyon, France.

\subsection{Ethoxy- and pentoxyresorufin 0-dealkylation assays - EROD/PROD} assays were carried out as described by Burke et al., 1985. Incubations were carried out at $370^{\circ} \mathrm{C}$ for $10 \mathrm{~min}$ in $0.1 \mathrm{M}$ potassium phosphate buffer $(\mathrm{pH} 7.8)$ and contained per $\mathrm{mL} 50$ $\mu \mathrm{g}$ microsomal protein, $1.6 \mathrm{mg} \mathrm{BSA}, 5 \mu \mathrm{M}$ substrate (ethoxy-or pentoxyresorufin) and a NADPH-regenerating system (consisting of $0.4 \mu \mathrm{mol} \mathrm{NADP+}, 5 \mu \mathrm{mol} \mathrm{MgCl}_{2}, 5 \mu \mathrm{mol}$ DL-isocitrate and 0.65 units of isocitric dehydrogenase). The incubation volume was 1.25 $\mathrm{mL}$. The reaction was stopped by the addition of $2.5 \mathrm{~mL}$ methanol (total volume 3.75 ), the mixture was centrifuged for $3 \mathrm{~min}$ (bench top centrifuge, at 2,000 rpm), and the 
flourescence due to the formation of resorufin in the clear supernatant was measured at excitation wavelength of $350 \mathrm{~nm}$ and ernission wavelength of $585 \mathrm{~nm}$, excitation/emission slits $5 / 3 \mathrm{~nm}$ respectively (Instrument: Perkin-Elmer LS-5 spectrofluorimeter). Standard curve of resorufin was used for calculations.

\subsection{7 p-Amino hippurate (PAH) transport - Three animal groups were used for} this experiment: control rats, rats treated with OTA, and PB-pretreated rats treated with OTA. The latter two groups were rats used to study urinary enzymes $(n=4)$. PAH transport was measured in vitro as described by Berndt and Hayes (1979). Briefly, after animal sacrifice by cervical dislocation, kidneys were removed rapidly and placed in cold Krebs-Ringer phosphate buffer pH 7.4. The renal slices $(0.25-0.40 \mathrm{~mm})$ were prepared freehand and stored in the same Krebs-Ringer phosplate buffer until used. All incubations were performed in a shaking wa er bath at $25{ }^{\circ} \mathrm{C}$ in an atmosphere of $95 \%$ oxygen $/ 5 \% \mathrm{CO}_{2}$ and were initiated within $30 \mathrm{~min}$ of the preparation of the slices. $100.150 \mathrm{mg}$ of tissues from all groups were incubated for 10 and $30 \mathrm{~min}$ in $2 \mathrm{~mL}$ of Krebs-Ringer phosphatc buffer after the addition of [14 C]PAH $(2 \mu \mathrm{Ci}, 50 \mu \mathrm{M}$; ICN, Canada). At the end of the incubation, tissues were removed from the buffer, blotted, and homogenized in $1 \mathrm{~mL}$ of distilled water. $0.5 \mathrm{~mL}$ of the homogenate was solubilized in $4.5 \mathrm{~mL}$ NCS-tissue solubilizer (Amersham), and $1 \mathrm{~mL}$ of the solution was counted for radioactivity after the addition of scintillation liquid (Scintiverse). Also, $1 \mathrm{~mL}$ of the bathing solution was used to count its radioactivity. The uptake (transport) of $\mathrm{PAH}$ is presented as slice/medium (S/M) ratio, i.e., the radioactivity/gm of tissue divided by the radioactivity $/ \mathrm{mL}$ of bathing solution. 


\subsection{In vivo studies -}

\subsubsection{Urinary enzyme experiments -}

2.18.1 a Preparation of urine samples - Samples (whole $24 \mathrm{hr}$ urines) were centrifuged for $5 \mathrm{~min}$ at $4,000 \mathrm{rpm}$. The clear supernatants were removed carefully and used for analysis.

2.18.1b Gel filtration of urine samples - This was done according to the method described by Werner et al. (1969). Eight glass columis with small dead space (inner diameter $1 \mathrm{~cm}$, height $30 \mathrm{~cm}$ ) were each filled to a height of about $18 \mathrm{~cm}$ with hydrated Sephadex G-50, giving a gel bed of about $14 \mathrm{~cm}^{3}$ in each column. Separation was performed at room temperature, and physiological saline $(0.154 \mathrm{M} \mathrm{NaCl})$ was used as eluant. Each urine sample $(3 \mathrm{~mL})$ was washed into the column with $1 \mathrm{~mL}$ saline followed by another $1 \mathrm{~mL}$ of saline. The liquid emerging from the column up to that time $(5 \mathrm{~mL})$ was discarded. Saline was placed on each column, and $6 \mathrm{~mL}$ of eluates was collected for enzyme analysis. The columns were finaliy filled and rinsed with saline to prepare them for reuse.

2.18.1c Measurement of urinary enzymes - Alkaline phosphatase and $\gamma$-glutamyl transferase activitics were measured using kits (kits numbers 104-LL and 545-A respectively) from Sigma Chemical Co. (St. Louis, MO). To avoid experimental variations due to different urine volumes, the enzyme activities were expressed as units/mg creatinine (creatinine kit number 555-A, Sigma Chemical Co.).

2.18.2 [3H]OTA experiments - Rats were anaesthetized with diethyl ether ( $6 \mathrm{hr}$ after OTA treatment), and blood was withdrawn from the abdominal aorta. After sacrifice, kidneys, liver, stomach, small intestine and caecum were excised, and the small intestine (Int.) was divided into four equal parts by length (Int.1 to Int.4). Gut segments [stomach, small intestine (Int.1, Int.2, Int.3 and Int.4), and cecum] were rinsed twice with $1 \mathrm{~mL}$ portions of $0.5 \%$ sodium taurocholate in nomal saline and then twice with $1 \mathrm{~mL}$ portions 
of $50 \mathrm{mM}$ sodium bicarbonate to ensure complete removal of all contents which was kept for extraction. Tissues were homogenized in water using a polytron. Tissuc homogenates and contents were acidified to $\mathrm{pH} 2$ and extracted three times with chloroform ( $5 \mathrm{~mL}$ lots). After drying the combined extracts under nitrogen, samples were redissolved in $40 \%) \mu \mathrm{L}$ methanol, and $200 \mu \mathrm{L}$ samples were counted for radioactivity. Blood samples were allowed to stand at room temperature for at least $\mathrm{I} \mathrm{hr}$ and then centrifuged to obtain serum. Calculation of total serum volume was done assuming the blood volume ( $\mathrm{mL}$ ) is given by the numerical value of $7 \%$ of $n$ rat's mass (in $\mathrm{gm}$ ) and the serum volume to be $55 \%$ of blood volume. Samples of urine and serum $(50-100 \mu \mathrm{L})$ were also counted for radioactivity.

\subsection{Spectrophotometric/spectrofluorometric measurements -} Spectrophotometric measurements were conducted on a Perkin-Elmer lambda $3 \mathrm{~B}$ double beam spectrophoto-meter in cells with a $1 \mathrm{~cm}$ light path. Fluorescence measurements were made in a Perkin-Elmer LS-5 spectrofluorimeter in cells with a square $\mathrm{cm}$ section, $1 \mathrm{~cm}$ in each dimension.

\subsection{Statistical analysis -}

The non-parametric Mann-Whitney test was used for the analysis of results from in vivo experiments $(\mathrm{N}=4$ or 8$)$ and results from in vitro experiments where $N=4$. $\wedge$ difference at $P<0.05$ was considered to be statistically significant. For the rest of in virro experiments, the number of animals from which samples were used was tors small to he analysed. When an experiment was done on samples from two different animals from each treatment group, the results from each animal were given separately. When an experiment was done on more than two animal (three or four) per treatment group, the results were given as the mean \pm S.D. from all animals. 


\section{Figure 5 The UV spectrum of OTA.}

The continuous line represents standard OTA from Sigma Chemical Co. $(\mathrm{ST}, \rightarrow$, while the broken line represents my OTA preparation (OT, --). Both samples were at a concentration of $25 \mu \mathrm{M}$ OTA in methanol. The instrument (Perkin Elmer Lambda 3B spectrophotometer) settings were: wavelength scan $400-200 \mathrm{~nm}$, chart speed $60 \mathrm{~mm} / \mathrm{min}$ and scan speed 60 $\mathrm{nm} / \mathrm{min}$. 


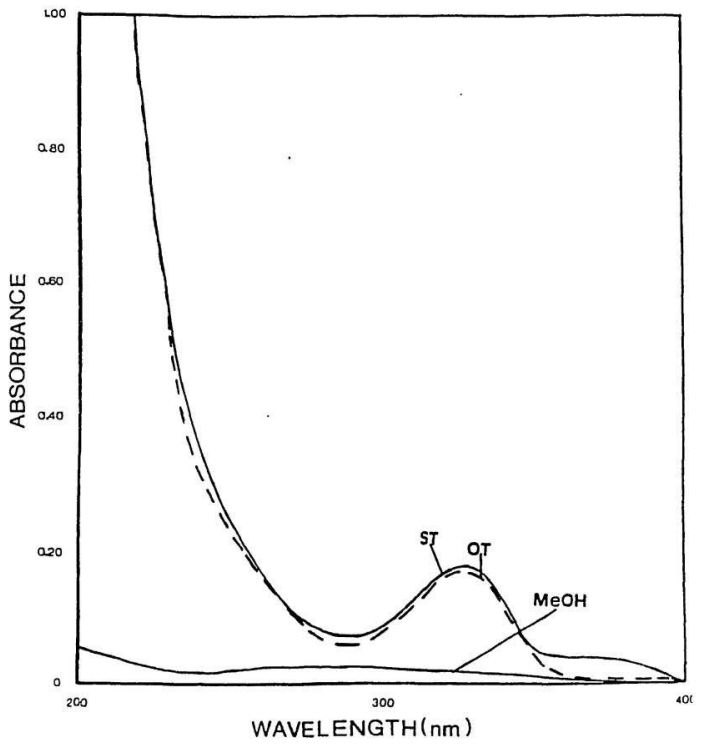


Figure 6 The nuorescence spectrum of OTA.

The continuous line represents standard OTA from Sigma Chemical Co. $(\mathrm{ST},-)$, while the broken line represents my OTA preparation (OT, -.). Both samples were at a concentration of $50 \mu \mathrm{M}$ OTA in methanol. The instrument (Perkin Elmer LS-5 spectrofluorimeter) settings were as follows: Excitation wavelength $340 \mathrm{~nm}$, while emission was scanned between 300 and $500 \mathrm{~nm}$. The excitation/emission slits were set at $5 / 3$ $\mathrm{nm}$, chart speed $60 \mathrm{~mm} / \mathrm{min}$ and scan speed $120 \mathrm{~nm} / \mathrm{min}$. 


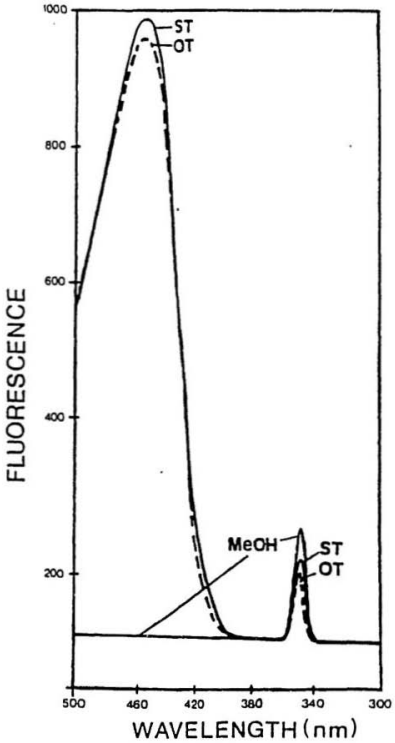


Table 6. The $R_{f}$ values of ochratoxin $A$.

\begin{tabular}{lcc}
\hline Solvent system & Sigma OTA* & Prepared OTA* \\
\hline $\begin{array}{l}\text { Benzene : acetic acid } \\
(4: 1, v / v)\end{array}$ & 0.68 & 0.68 \\
Benzene : acetic acid & 0.41 & 0.41 \\
$(8: 1, v / v)$ & & \\
Benzene : acetic acid : Methanol & 0.45 & 0.45 \\
$(95: 5: 5, v / v / v)$ & & \\
\end{tabular}

* $3 \mu \mathrm{L}$ of $25 \mathrm{mM}$ were spotted on TLC plates with fluorescent indicator. 
Figure 7 The HPLC profile of our OTA preparation.

Sample $(20 \mu \mathrm{L}$ of $25 \mu \mathrm{M}$ solution in methanol) was injected into a Perkin Elmer-Series 4 liquid chromatograph. The column used was Partisil 10 ODS-2. The solvent system consisted of a mixture of a) acetonitrile:methanol ( $500: 500$, v/v) $65 \%$ and b) $5 \mathrm{mM}$ sodium acctate:acetic acid (500:14, v/v) $35 \%$. The flow rate was $1.5 \mathrm{~mL} / \mathrm{min}$ and the fluorimetric detection was performed at excitation wavelength of 340 $\mathrm{nm}$, emission wavelength of $465 \mathrm{~nm}$ and excitation/emission slits of $5 / 10$ $\mathrm{nm}$. 
Detecror Response (mvolts)

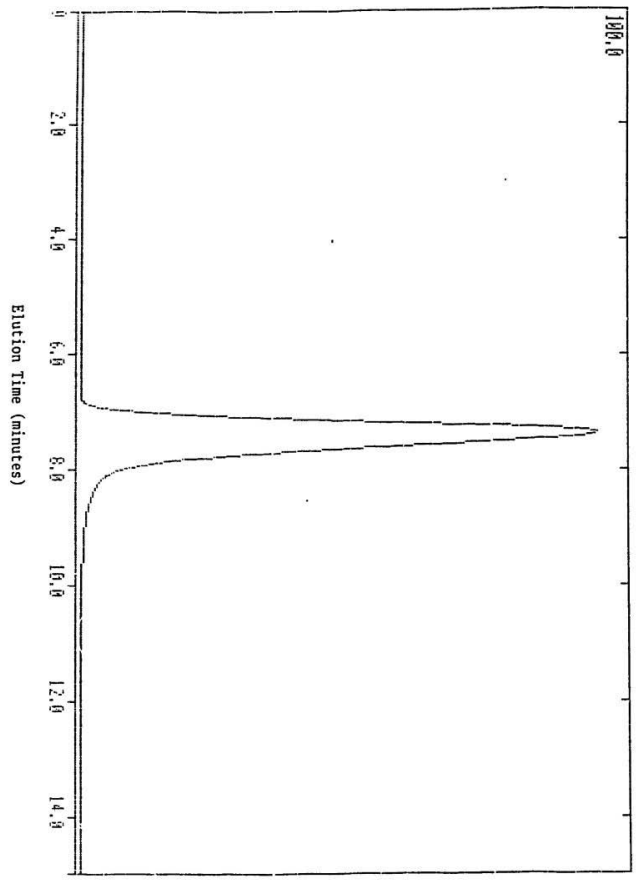


Figure 8 Proton NMR spectrum of my OTA preparation. 


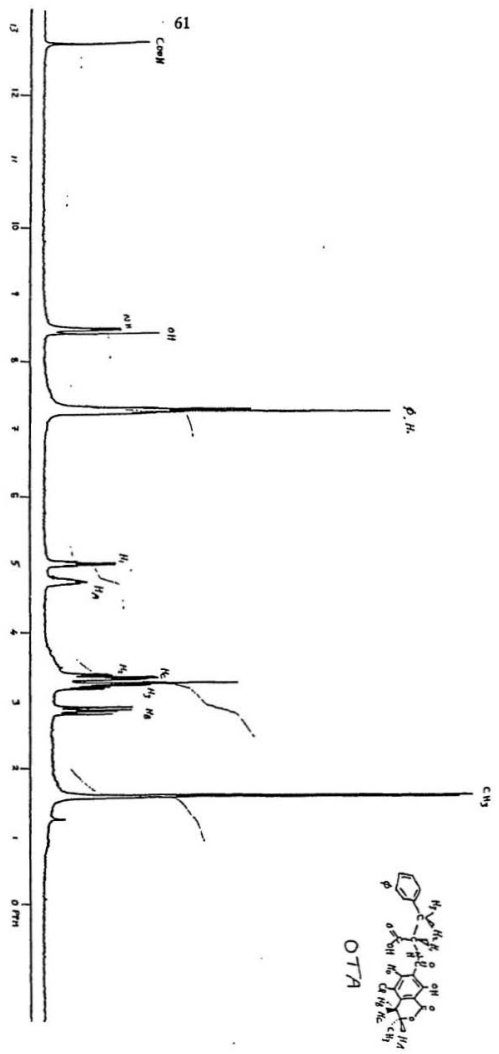


Table 7. List of various treatments and cytochromes $P-450$ induced.

\begin{tabular}{|c|c|c|c|}
\hline Treatment & $\begin{array}{l}\text { Major P-450 } \\
\text { Enz. Induced }\end{array}$ & $\begin{array}{l}\text { Route of Administration, Dose } \\
\text { and Vehicle }\end{array}$ & Reference \\
\hline Control & - & No treatment. & - \\
\hline PB & IIB1. & $0.1 \%$ in drinking water for 5 days. & Graves, 1987 \\
\hline 3-MC & IAI & $\begin{array}{l}20 \mathrm{mg} / \mathrm{Kg} \text { in com oil i.p. once each day } \\
\text { for } 3 \text { successive days. }\end{array}$ & Guengerich, 1982 \\
\hline Clofibrate & IVA1 & $\begin{array}{l}200 \mathrm{mg} / \mathrm{Kg} \text { in } 1 \mathrm{~mL} \text { sucrose syrup containing } \\
1 \% \text { arabic gum given by gastric intubation } \\
\text { once each day for } 5 \text { days. }\end{array}$ & Fournel, 1987 \\
\hline Isosafrole & IA1/LA2 & $\begin{array}{l}120 \mathrm{mg} / \mathrm{Kg} \text { in corn oil i.p. once each day } \\
\text { for } 3 \text { successive days. }\end{array}$ & Fischer, 1981 \\
\hline $\mathrm{PCN}$ & ША1/ША2 & $\begin{array}{l}100 \mathrm{mg} / \mathrm{Kg} \text { in } 1 \mathrm{~mL} 1 \% \text { Tween } 80 \text { given by } \\
\text { gastric intubation once each day for } 4 \text { days. }\end{array}$ & Graves, 1987 \\
\hline Isoniazid & IIE1 & $0.1 \%$ in drinking water for 10 days ( $\mathrm{pH} 7.4)$. & Ryan, 1985 \\
\hline
\end{tabular}

$N=4 /$ treatment group. 


\section{CHAPTER 3}

\section{RESULTS}

\subsection{Role of EDTA and cytochrome P-450 in the stimulation of OTA- induced lipid peroxidation in reconstituted systems.}

In a reconstituted microsomal lipid peroxidation system consisting of microsomal phospholipid, Fp, Fe3+, OTA and NADPH (see Methods section 2.14.1), the concentrations of MDA obtained were $1.65 / 2.20$ and $2.46 / 4.60 \mathrm{nmol} / \mathrm{mL}$. at the end of 20 and $40 \mathrm{~min}$ respectively (values from two different experiments). In this experiment, purified cytochrome $\mathrm{P}-450$ and Fp (from 6-8 rats each) not microsomes from individual rats were used. Hence, experiments 1 and 2 were performed on two different days (each in duplicate) but using the same purified enzymes (as these were isolated only once). Addition of EDTA ( $25 \mathrm{nmol} / \mathrm{mL}$ ) to the reconstituted system led to a three- to four-fold increase in the concentration of MDA formed yielding $6.62 / 7.45$ and $10.24 / 13.60 \mathrm{nmol}$ $\mathrm{MDA} / \mathrm{mL}$ at 20 and $40 \mathrm{~min}$ respectively (Table 8 ). Replacement of EDTA by purified cytochrome P-450 IIB 1 also led to a stimulation of lipid peroxidation. Figure 9 shows the extent of this stimulation in the presence of increasing concentrations of cytochrome P-450 IIB1. Concentrations up to $0.3 \mathrm{nmol}$ cytochrome $\mathrm{P}-450 / \mathrm{mL}$ had a stimulatory effect on MDA production, which amounted to $6.5 / 8.5$ and $10 / 13 \mathrm{nmol} M D A$ at the end of 20 and 40 min respectively. Intact cytochrome P-450 sceris to be essential since the heatdenatured enzyme, hematin, or a variety of hemoproteins were all ineffective in stimulating lipid peroxidation (Table 9), but carbon monoxide (CO) did not inhibit the stimulatory effect of cytochrome P-450. It is clear that the addition of only cytochrome P-450, but not other heme or hemoproteins stimulated lipid peroxidation (in this experiment, purified cytochrome P-450 from 6-8 rats not microsomes from individual rats was used). 


\subsection{Effect of microsomes from Co-heme pretreated rats on OTA-stimulated lipid peroxidation.}

This was studied as described in Methods sections 2.5.2, 2.12 and 2.14.2. The data in Figure 10 suggest that cytochrome P-450 may also be involved in OTA-dependent microsomal lipid peroxidation. The rate of MDA formation was highest in microsomes isolated from untreated rats. Thus, at the end of a 10-min incubation period, microsomes from untreated rats produced 7.6/9.4 nmol of MDA (values from two different experiments). In contrast, microsomes isolated from $\mathrm{Co}$-heme-pretreated rats showed a much lower rate of lipid peroxidation which, at $10 \mathrm{~min}$, amounted to only $2.2 / 2.5 \mathrm{nmol}$ of MDA. Treatment of rats with Co-heme has been shown to lower hepatic cytochrome P450 levels (Drummond and Kappas, 1982; Spaethe and Jollow, 1989), and also to reduce Fp activity (Spaethe and Jollow, 1989). Microsomes from Co-heme-pretreated rats contained $0.6 \mathrm{nmol}$ of cytochrome $\mathrm{P}-450$ and 22 units of $\mathrm{Fp}$ per $\mathrm{mg}$ protein. These values are $20 \%$ and $25 \%$ of the respective values in control microsomes (Table 10). To establish that the greatly reduced rate of MDA formation by microsomes from Co-heme-preireated rats was not due primarily to lower Fp levels, microsnmes from $\mathrm{Co}$-heme-pretreated rats fortified by Fp were examined for their ability to carry out OTA-dependent lipid peroxidation. Fp fortified microsomes contained $78 \%$ of Fp of control microsomes. The results show that Fp-fortification of microsomes from Co-heme-pretreated rats only marginally increased MDA formation to $3,4 / 4.1 \mathrm{nmol}$ at $10 \mathrm{~min}$.

\subsection{Effect of various cytochrome P-450 inducers on OTA metabolism by liver microsomes.}

The structures of OTA and its metabolites 4(R)- and 4(S)-4-OH-OTA are shown in Figure 1. Figure 11 shows the HPLC profile of OTA and its metabolites. Table 7 lists the various methods of inducing cytochrome P-450 (Methods section 2.5.1), together with the 
major isoforms of cytochrome P-450 induced (the unified nomenclature by Nebert et al., 1987 is used). The effect of these inducers on the hepatic cytochrome P.450 concentration in liver microsomes is shown in Table 11. Some of the cytochrome P-450 inducers increased the total cytochrome P-450 concentration (e.g. PB, 2.5 fold increase over control; 3MC, 2 fold; CLF and PCN, about 1.7 fold and ISF, about 1.2 fold) while other only induced specific cytochrome P-450 isozymes without increasing the total content (e.g. INH). The extent of OTA metabolism by microsomes from rats treated with the cytochrome $\mathrm{P}-450$ inducers is shown in Figure 12. After the induction of cytochrome P450 in liver with 3MC, ISF, PB and PCN, the microsomal metabolism of OTA in vitro (Methods section 2.15.1) gave 4(R)-4-OH-OTA as the major metabolite. After induction with CLF both $4(R)$ and $4(S)$ isomers were produced in increased but equal amounts, and after induction with INH the $4(\mathrm{~S})$ isomer predominated. Results from a completely different batch (Batch II) are shown in Figure 13. Figure 14 shows the effect of vehicle controls of the different inducers of cytochrome P-450 on the metabolism of OTA.

\subsection{Substrate specificity of cytochromes P-450 IIB1 and IA1/IA2.}

The substrates 7-pentoxyresonfin (specific for PB inducible cytochrome P-450 IIB1, Lubet et al., 1985) and 7-ethoxyresorufin (specific for 3MC-inducible cytochrome P$4501 A 1 / 1 A 2$, Burke and Mayer, 1983) showed selective or preferential reactions with the appropriate cytochrome P-450 isoforms. Thus, liver microsomes from PB-treated rats dealkylated 7-pentoxyresorufin about 30 times more rapidly than those from control rats, and liver microsones from 3MC-treated rats dealkylated it at a rate of only about 4.5 times that of microsomes from control animals. In contrast, microsomes from $3 \mathrm{MC}$ treated rats dealkylated 7-ethoxyresorufin about 82 / 88 times as rapidly as those from controls, and microsomes from PB treated rats increased its rate of metabolism only about 10.5/20 fold over controls (Table 12). 


\subsection{Effect of specific inhibitors of cytochromes P-450 IA1/IA2 and IIBI on the metabolism of OTA in vitro.}

Figure 15 shows the effect of $\alpha$-naphthonavone (a known inhibitor of cylochrome P-450 IA 1/2, Wiebel et al., 1971) and metyrapone (a known inhibitor of cytochrome P-450 IIB1, Jonen et al., 1974) on the metabolism of OTA to 4(R)-4-OH-OTA by microsomes from PB, 3MC, and ISF treatments. $\alpha$-Naphthoflavone selectively inhibited the formation of $4(\mathrm{R})$ isomer by microsomes from rats pretreated with $3 \mathrm{MC}$ and ISF $(\sim 89 / 95 \%$ inhibition in both cases; results from two different experiments). The inhibition was $\sim 18$ / $41 \%$ using microsomes from rats treated with $\mathrm{PB}$. On the other hand, metyrapone inhibited the formation of $4(R)$ isomer by microsomes from rats treated with $\mathrm{PB}$ ( 94/ $96 \%$ inhibition), and also inhibited (to a lesser extent) its formation ( $40 / 11 \%$ and $\sim 40$ / $16 \%$ inhibition) by microsomes from $3 \mathrm{MC}$ and ISF treated rats respectively (Figure 15). The effect of the two inhibitors on 4(S) isomer formation was less selective (Figure 16).

\subsection{Effect of monoclonal antibodies (MAbs) against cytochromes P-450 on the metabolism of OTA by liver microsomes in vitro.}

Two main monoclonal antibodies were used: clone 1-7-1 against cytochrome P-450 IA1/IA2 and clone 2-66-3 against cytochrome P-450 IIB 1/IIB2. The preincubation of liver microsomes from rats pretreated with $3 \mathrm{MC}$, with monoclonal antibody 1-7-1 reduced the subsequent formation of 4(R)-4-OH-OTA from OTA in vitro (Figure 17a). At a MAb to microsomal protein ratio of 5 , the formation of the 4 (S)-isomer was also partially inhibited (Figure 17a). Rabbit IgG was used to determine the non specific inhibition of OTA metabolism in vitro. The preincubation of liver microsomes from rats pretreated with $3 \mathrm{MC}$, with rabbit IgG slightly inhibited the formation of the $4(R)$-isomer only at IgG to microsomal protein ratio of 5 (Figure 17b). Preincubating liver microsomes from rats treated with PB, with MAb 2-66-3 reduced the subsequent formation of 4(R)-4.OH-OTA 
from OTA in vitro (Figure 18a). At MAb to microsomal protein ratios of 2, 3 and 5, the formation of the $4(\mathrm{~S})$ - isomer was also reduced (Figure 18a). The non specific inhibition of OTA metabolism resulting from preincubating rabbit IgG with liver microsomes from PB treated rats is shown in Figure 18b. Table 13 shows the cross reactivity of each of the two monoclonal antibodies with microsomes from rats treated with PB and $3 \mathrm{MC}$. With respect to inhibition of the $4(\mathrm{R})$-isomer formation, MAb 2-66-3 showed greater specificity towards microsomes from PB-treated rats whereas MAb 1-7-1 showed greater specificity towards microsomes from $3 \mathrm{MC}$ treated rats (Table 13). However, both antibodies especially 2-66-3 showed substantial cross-reactivity. MAbs against cytochrome P-450 enzymes are very expensive; if they were to be purchased, the cost would have been several thousand dollars. the fact that we obtained them as a gift and in small quantity, we were not able to repeat these experiments again.

\subsection{Time course of OTA metabolism by liver microsomes from rats treated with INH and PCN.}

OTA metabolism by liver microsomes from untreated (control) rats was very poor giving rise to $4(\mathrm{R})-4-\mathrm{OH}-\mathrm{OTA}$ and $4(\mathrm{~S})-4-\mathrm{OH}-\mathrm{OTA}$ levels that averaged 0.015 and 0.014 nmol/mg protein respectively over a $30 \mathrm{~min}$ incubation period (Table 14). Microsomes from INH-treated rats were chosen to further study the $4(\mathrm{~S})$-isomer formation since this pretreatment induces $4(\mathrm{~S})$-isomer formation (about 7 fold) without concomitantly increasing 4(R)-isomer formation. Microsomes from PCN-trated rats were selected to study $4(R)$-isomer formation since this pretreatment increased 4(R)-4-OH-OTA formation 55-fold over control microsomes. PCN pretreatment also increased 4(S)-isomer formation by 10 -fold over control microsomes (Table 14).

Figure 19 shows the time course of OTA metabolism and lipid peroxidation using microsomes from INH-pretreated rats. There was very little 4(R)-4-OH-OTA formation 
which reached a maximun of $0.017 \mathrm{nmol} / \mathrm{mg}$ protein at $40 \mathrm{~min}$. In contrast, 4(S)-4-OH-OTA formation continued to increase steadily reaching a maximum of 0.080 $\mathrm{nmol} / \mathrm{mg}$ protein at $40 \mathrm{~min}$, the final time point tested (Figure 19a). In parallel measurements, MDA formation also increased steadily reaching $16 \mathrm{nmol} / \mathrm{mg}$ protein by 40 $\min$ (Figure 19b). The time course of OTA metabolism and lipid peroxidation by microsomes from PCN-pretreated rats is shown in Figure 20. In contrust to microsomes from INH-treated rats, microsomes from PCN-treated rats efficiently catalyzed the formation of 4(R)-4-OH-OTA which steadily increased to about $1 \mathrm{nmol} / \mathrm{mg}$ protein al 40 $\mathrm{min}$, the final time point tested. 4(S)-4-OH-OTA formation also increased steadily with time to reach $0.17 \mathrm{nmol} / \mathrm{mg}$ protein by $40 \mathrm{~min}$ (Figure 20a). As in the case of microsomes from INH-treated rats, MDA formation also increased with time reaching $20.5 \mathrm{nmol} / \mathrm{mg}$ protein at $\mathbf{4 0} \mathrm{min}$ (Figure $20 \mathrm{~b}$ ).

\subsection{Effect of pH on OTA metabolism and /,pid peroxidation.}

The $\mathrm{pH}$ optima for the formation of 4(S)-4-OH-OTA (Figure 21a) and lipid peroxidation (Figure 21b) by microsomes from INH-treated rats were 7.0 and 6.5 - 7.5 respectively. $4(\mathrm{R})$ did not show a very distinct $\mathrm{pH}$ optimum, possibly because of the very low levels produced. The $\mathrm{pH}$ optima for the formation of $4(\mathrm{R})-4-\mathrm{OH}-\mathrm{OTA}$ (Figure 22a) and lipid peroxidation (Figure 22b) by microsomes from PCN-treated rats were 6.5 and 7.0 - 7.5 respectively. $4(S)$ did not show a distinct pH optimum, possibly because of low levels of production.

\subsection{Effect of active oxygen seavangers, antioxidants and iron chelators on} OTA metabolism and lipid peroxidation.

Tables 15 and 16 shows the effect of superoxide dismutase (SOD), catalase, mannitol, butylated hydroxyanisole (BHA), N,N-diphenyl-1,4-phenylenediamine (DPPD), 
bathophenanthrolinedisulfonic acid (BPS) and Desferal addition on OTA metabolism and lipid peroxidation by microsomes from $\mathrm{INH}$ - and $\mathrm{PCN}$-pretreated rats. With microsomes from INH-pretreated rats, 4(S)-4-OH-OTA formation was inhibited $100 \%$ by BHA, over $90 \%$ by DPPD, Desferal and BPS, about $30 \%$ by mannitol and about $15 \%$ by SOD and catalase. In contrast, none of these agents inhibited 4(R)-4-OH-OTA formation with the exception of DPPD which exerted about $40 \%$ inhibitory effect. In fact, both Desferal and BPS increased the yield of $4(\mathrm{R})-4-\mathrm{OH}-\mathrm{OTA}$ by $60-170 \%$ with microsomes from both sources (Table 16). Parallel determinations of lipid peroxidation indicated that BHA, DPPD, Desferal and BPS all inhibited MDA formation by $>90 \%$, mannitol was slightly inhibitory (15\%) while catalase and SOD had no effect.

When microsomes from $\mathrm{PCN}$ treated rats were used, both antioxidants (BHA and DPPD) inhibited 4(S)-4-OH-OTA formation by 50\% while BPS exerted a $35 \%$ inhibitory effect, but SOD, catalase and Desferal increased $4(\mathrm{~S})-4-\mathrm{OH}-\mathrm{OTA}$ levels by $35 \%, 50 \%$ and $30 \%$ respectively; mannitol was without effect. $4(\mathrm{R})-4-\mathrm{OH}-\mathrm{OTA}$ formation was not affected except by catalase, Desferal and BPS which increased it by about $30 \%, 100 \%$ and $170 \%$ respectively. As expected, MDA formation was strongly inhibited by BHA, DPPD, Desferal and BPS ( $>90 \%$ ) while both catalase and mannitol were mildly inhibitory (15 $20 \%$ ). Figure 23 shows that varying concentrations of BHA inhibited to roughly the same extent both 4(S)-4-OH-OTA formation and lipid peroxidation by liver microsomes from INH treated rats. Thus, $2.5 \mu \mathrm{M}$ and $5 \mu \mathrm{M}$ BHA were required to completely inhibit 4(S)-4-OH-OTA formation at $10 \mathrm{~min}$ and $30 \mathrm{~min}$ respectively (Figure 23a), and $5 \mu \mathrm{M}$ BHA was required to inhibit MDA formation (Figure 23b) at $10 \mathrm{~min}$ and $30 \mathrm{~min}$. Lower concentrations of BHA were correspondingly less inhibitory. In contrast, 4(R)-4 OH-OTA formation was not inhibited (data not shown; see also Tables 16 and 17).

Incubation of liver microsomes from INH- or PCN-treated rats with NADPH and OTA for 30 min resulted in the destruction of cytochrome $\mathrm{P}-450$ by $40 \%$ and $75 \%$ 
respectively. Inclusion of Desferal provided complete protection against cytochrome P-450 loss in the case of microsomes from INH-treated animals, and reduced the cytochrome P. 450 loss from $75 \%$ to $37 \%$ in the case of microsomes from PCN-treated animals (Table 16).

Replacement of NADPH by ascorbate/ $\mathrm{Fe}^{2+}$ almost virtually eliminated 4(R)-4-OH-OTA formation by microsomes from PCN-treated mats, but did not affect 4(S)-4-OH-OTA formation by microsomes from either PCN- or INH-trented rats (Table 17). As expected, the inclusion of BHA strongly inhibited 4(S)-4-OH-OTA formation as well as MDA formation ( $-90 \%)$ by microsomes from both sources. Addition of mannitol did not appreciably change the formation of 4(S)-4-OH-OTA, 4(R)-4-OH-OTA or MDA.

\subsection{Effect of various hemoproteins and hydroperoxides on OTA} metabolism.

Cumene hydroperoxide (CHP, $0.5 \mathrm{mM}$ ) could effectively replace NADPH in catalyzing the formation of 4(R)-4-OH-OTA and 4(S)-4-OH-OTA by liver microsomes from $\mathrm{PCN}$-treated rats ( $2 \mathrm{mg}$ microsomal protein $/ \mathrm{mL}$ ) giving 0.53 and $0.03 \mathrm{nmol}$ metabolite/nmol heme respectively (Table 18$)$. By comparison, $\mathrm{H}_{2} \mathrm{O}_{2}(5 \mathrm{mM})$ was much less effective giving only 0.015 and $0.013 \mathrm{nmol}$ of the $4(\mathrm{R})$ - and $4(\mathrm{~S})$-isomers respectively, while linoleic acid hydroperoxide (LAHP, $65 \mu \mathrm{M}$ ) was totally ineffective. When microsomes were replaced with other hemoproteins e.g, hemoglobin or HRP (20) $\mathrm{nmol} / \mathrm{mL}$ ), or by hematin $(5 \mathrm{nmol} / \mathrm{mL}$ ) no OTA metabolism was observed in the presence of a variety of hydroperoxides (CHP, $0.5 \mathrm{mM} ; \mathrm{H}_{2} \mathrm{O}_{2}, 5 \mathrm{mM}$ or LAHP, $65 \mu \mathrm{M}$ ) (Table 18). 


\subsection{Effect of pH on OTA metabolism and lipid peroxidation by liver microsomes, and on cytochrome P-450 content of these microsomes.}

The $\mathrm{pH}$ optima for the formation of 4(R)-4-OH-OTA and 4(S)-4-OH-OTA, and for lipid peroxidation in liver microsomes from rats given different treatments are given in Figures 22,24 and 25 . The $\mathrm{pH}$ optimum for the formation of $4(\mathrm{R})$-isomer was 6.0 using microsomes from rats treated with 3MC (Figure 25) and ISF (data not shown), and 6.5 using microsomes form rats treated with PB (Figure 24a) and PCN (Figure 22). The pH optimum for the formation of $4(\mathrm{~S})$-isomer was 7.0 (Figures $21 \mathrm{a}, 24 \mathrm{a}$ and 25 ), which coincided with that for lipid peroxidation (Figures $21 \mathrm{~b}, 22 \mathrm{~b}$ and $24 \mathrm{~b}$ ). Table 19 shows a comparison between the effect of $\mathrm{pH} 6.0$ and 7.4 on OTA metabolism using microsomes from rats treated with $3 \mathrm{MC}$, ISF and PB. The formation of $4(\mathrm{R})$-isomer increased at $\mathrm{pH}$ 6.0 (compared to $\mathrm{pH} 7.4$ ) by 7.7- and 5.0-fold using liver microsomes from $3 \mathrm{MC}$ and ISF treated rats respectively. At $\mathrm{pH} 7.4$ (compared to $\mathrm{pH} 6.0$ ), the formation of $4(\mathrm{~S})$-isomer increased 3.4- and 2.7-fold using liver microsomes from ISF and PB treated rats. Table 20 shows the MDA formed and cytochrome P-450 contents at the end of 30 min incubation using liver microsomes from rats treated with $3 \mathrm{MC}, 1 \mathrm{SF}$ and $\mathrm{PB}$ at $\mathrm{pHs} 6.0$ and 7.4. More MDA and lower cytochrome $\mathrm{P}-450$ contents were observed at $\mathrm{pH} 7.4$ as compared to $\mathrm{pH}$ 6.0. Thus, MDA formed at $\mathrm{pH} 7.4$ was $26.8,17.1$ and $20.2 \mathrm{nmol} / \mathrm{mg}$ protein using liver microsomes form rats treated with $3 \mathrm{MC}$, ISF and PB as compared to $18.9,8.0$ and 11.8 $\mathrm{nmol} / \mathrm{mg}$ protein respectively at $\mathrm{pH} 6.0$ for microsomes from the same sources. At $\mathrm{pH}$ 7.4, the percentage loss of cytochrome P-450 contents was 57,43 and 59 using liver microsomes form rats treated with $3 \mathrm{MC}$, ISF and $\mathrm{PB}$ as compared to 32,15 and 51 respectively at $\mathrm{pH} 6.0$ for microsomes from the same sources (Table 20). It seems that the loss in cytochrome P.450 at pH 6.0 for microsomes from $3 \mathrm{MC}$ and ISF treated rats is not as much as that for microsomes from PB treated rats. This may, partly, explain the 5.0and 7.7 -fold increase in the $4(\mathrm{R})$ isomer formation (at $\mathrm{pH} 6.0$ ) using microsomes from ISF 
and $3 \mathrm{MC}$ treated rats, and the 1.8-fold increase using microsomes from $\mathrm{PB}$ treated rats (Table 19). It also seems that the loss in cytochrome P-450 is greater when there is more lipid peroxidation (i.e. at $\mathrm{pH} 7.4$ compared to $\mathrm{pH} 6.0$, Table 20 ).

3,12 Effect of $\mathrm{pH}$ on the $\mathrm{Km}$ and Vmax of OTA hydroxylase in liver microsomes from rats treated with $3 \mathrm{MC}$ and ISF.

The $\mathrm{Km}$ for OTA hydroxylation (measured at $\mathrm{pH} 7.4$ ) was calculated to be $74 \mu \mathrm{M}$ for microsomes from rats treated with $3 \mathrm{MC}$ (Figure 26a), whereas the Vmax was 38 $\mathrm{pmol} / \mathrm{min} / \mathrm{mg}$ protein. At pH $6.0, \mathrm{Km}$ dropped to $31 \mu \mathrm{M}$ and $\mathrm{Vmax}$ increased to 195 $\mathrm{pmol} / \mathrm{min} / \mathrm{mg}$ protein (Figure 26b). The $\mathrm{Km}$ and Vmax for OTA hydroxylation (measured at $\mathrm{pH} 7.4$ ) by microsomes from rats treated with ISF were $92 \mu \mathrm{M}$ and $13 \mathrm{pmol} / \mathrm{min} / \mathrm{mig}$ protein (Figure 27a). The corresponding values at $\mathrm{pH} 6.0$ werc $150 \mu \mathrm{M}$ and 279 $\mathrm{pmol} / \mathrm{min} / \mathrm{mg}$ protein (Figure $27 \mathrm{~b}$ ).

\subsection{In vivo experiments.}

\subsubsection{Effect of PB treatment on OTA-induced release of enzymes in the urine.}

Figure 28 shows urine alkaline phosphatase levels both in PB-treated and control ral groups on day 0 (before OTA treatment) and days 1-5 of OTA treatment. Enzyme level was elevated significantly (compared to base line, day 0 ) on the fourth day of OTA treatment in the control group (four fold increase), whereas there was no increase in enzyme level for the PB-treated group. In case of $\mathbf{Y}$-glutamyl iransferase, enzyme levels in the urine increased on days $1,2,3$ and 4 of OTA treatment in the control group by 2.5., 2.0-, 1.7- and 3.5-fold (compared to base line, day 0). For the PB group, enzyme levels increased at days 2 and 3 by 2.7 - and 2.6-fold (Figure 29). 
Transport of the anion p-amino hippurate (PAH) in kidney cortex slices obtained from $\mathrm{PB}$-pretreated rats treated with OTA (PB + OTA) and rats treated with OTA (OTA) was significantly inhibited to the same extent $(55 \%-60 \%)$ as compared to control animals (C) (Figure 30). However, there was no significant difference between the two groups (OTA and PB + OTA) in terms of their inhibition of PAH transport (Figure 30).

\subsubsection{Effect of PB treatment on the distribution of OTA.}

Figure 31 shows the percentage of administered [ $\left.{ }^{3 \mathrm{H}}\right]$ OTA found in serum and urine of PB-treated and control rats $6 \mathrm{hr}$ after OTA administration. Even though it was not significantly different, there was a tendency of increased OTA concentration in the serum of control (12.4\% of administered dose) as compared to PB-treated rats (8.4\%), whereas there was an increase in OTA concentration in the urine of PB treated rats $(5.2 \%)$ as compared to control rats (2.0\%). OTA levels in the liver (1.5\% for control group and $1.24 \%$ for the PB-treated group) and the kidney $(0.15 \%$ for control group and $0.13 \%$ for PB group) did not show a significant difference between the two groups (Figure 32). The percentage of the toxin accumulated in the whole organ is more in the liver $(1.2 \%-1.5 \%)$ as compared to the kidney $(0.13 \%-0.15 \%)$, but on a per gram basis, more toxin accumulated in the kidney than in the liver. This is in agreement with the finding of Galtier et al. (1979). The percentage of administered [3H]OTA in the gut tissues (stomach, the four portions of small intestine and cecum) are shown in Figure 33. The levels of OTA in the gut contents are shown in Figure 34. Higher OTA levels were found in the small intestinal tissuc ( $2-6$ fold increase) and gut contents ( $4-8$ fold increase) of control rats than of $\mathrm{PB}$ rats. 
Table 8. Involvement of EDTA or cytochrome P-450 in the stimulation of lipid peroxidation by OTA in a reconstituted system.

Addition to system

MDA formed
$(\mathrm{nmol} / \mathrm{mL})$

$20 \mathrm{~min}$

Exp.1 Exp.2 Exp.1 E E

None

1.65

2.20

2.46

4.60

EDTA $(25 \mathrm{nmol} / \mathrm{mL})$

6.62

7.45

10.24

13.6

EDTA (50

$\mathrm{nmol} / \mathrm{mL}$ )

5.14

6.30

7.96

11.2()

Cyt. P-450 (0.3 nmol $/ \mathrm{mL})$

4.30

6.20

7.11

10.03

Cyt. P-450 (0.5 nmol/mL)

5.35

4.40

8.45

9.78

Incubations were carried out as described in the Methods section 2.14.1. Each of the two experiments were carried out in duplicate. MDA values are means of the two separate incubations. 
Figure 9 Effect of cytochrome P-450 concentration on OTA stimulated lipid peroxidation.

Incubations were carried out in duplicate at $37{ }^{\circ} \mathrm{C}$ for 20 and $40 \mathrm{~min}$ in $0.25 \mathrm{M} \mathrm{Tris}-\mathrm{HCl}$ buffer, $\mathrm{pH} 6.8 / 0.25 \mathrm{M} \mathrm{NaCl}$ and contained per $\mathrm{mL}$ : phospholipid vesicles ( $1 \mu \mathrm{mol}$ P), $177 \mathrm{ng}$ Fp (3.2 units), 500 nmol OTA. varying amounts of cytochrome P-450 (0-0.3 nmol), $110 \mathrm{nmol} \mathrm{Fe} 31$ and $200 \mathrm{nmol}$ NADPH. The reaction volume was $1 \mathrm{~mL}$. Results of two separate experiments ( $A$ and $B$ ) are shown. 
76
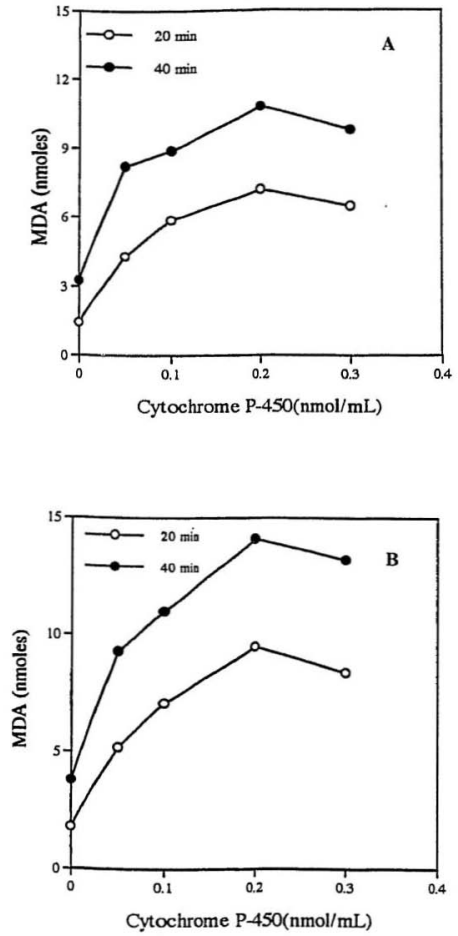
Table 9. Requirement for cytochrome P-450 in NADPH-dependent lipid peroxidation.

Hemoprotein/Treatment

MDA formed

( $\mathrm{nmol} / \mathrm{mL}$ )

None

1.98

Cyt. P-450

7.56

Cyt. P-450 (heat denatured) 1

2.02

Cyt. P-450 + carbon monoxide 2

7.33

Hematin

2.12

Hemoglobin

2.67

Cyt. $c$

2.43

Horseradish peroxidase

2.38

Incubations were carried out for $\mathbf{4 0} \mathrm{min}$ as described in the Methoo's section

2.14.1. Hematin and all hemoproteins were added in an amount corresponding to $0.3 \mathrm{nmol}$ heme/mL. MDA values are means from quadruplicate incubations that did not vary from each other by more than $10 \%$.

$1000 \mathrm{C}$ for $2 \mathrm{~min}$.

2 Carbon monoxide was bubbled through the incubation mixture for $30 \mathrm{sec}$ prior to addition of OTA. 
Figure 10 Effect of depleting cytochrome P-450 on OTA stimulated lipid peroxidation.

Incubations were carried out in duplicate at $376 \mathrm{C}$ for $0,5,20$ and $40 \mathrm{~min}$ in $0.1 \mathrm{M}$ phosphate buffer, $\mathrm{pH} 7.4$ and contained per mL: $2 \mathrm{mg}$ microsomal protein (from control or Co-heme treated rats), and $125 \mathrm{nmol}$ OTA. The reaction was initiated with $1 \mathrm{mM}$ NADPH (final concentration). The reaction volume was $1 \mathrm{~mL}$. Microsomes from rats treated with $\mathrm{Co}$-heme (before use in lipid peroxidation) were first fortified for Fp by incubating them with the purified enzyme preparation (as described is the Methods section 2.12. Results of two separate experiments ( $A$ and $B$ ) from two different animals are shown. 

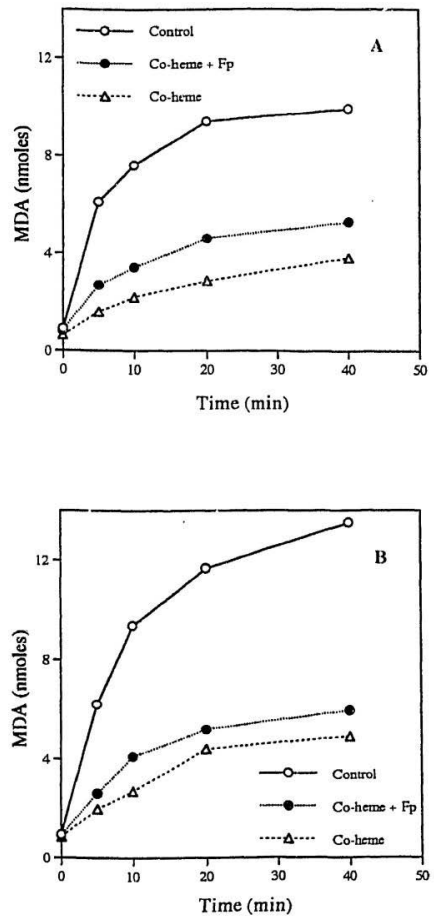
Table 10. Effect of Co-heme pretreatment on liver microsomal NADPH-cytochrome $\mathrm{P}-450$ reductase $(\mathrm{Fp})$ and cytochrome P-450 levels.

Treatment

P.450 Reductase 1

Cytochrome P-450 2

None

$88.2 \pm 7.3$

$2.92 \pm 0.31$

Co-heme

$22.3 \pm 2.7 *$

$0.60 \pm 0.05^{*}$

Co-heme + Fp

$70.1 \pm 6.8^{*}$

$0.57 \pm 0.05^{*}$

Animals received either $\mathrm{Co}$-heme ( $50 \mu \mathrm{mol} / \mathrm{kg}$ body weight; sub-cutaneously) or saline 9 days and 2 days prior to being sacrificed. NADPH-cytochrome P-450 reductase and cytochrome P-450 levels were determined as described in the Methods sections 2.11 and 2.9. Results are means \pm S.D of values from four individual rats/treatment group. ${ }^{*}$, means significantly different from no treatment $(p<0.05$, Mann Whitney test).

$1 \mathrm{nmol}$ cytochrome $c$ reduced $/ \mathrm{min} / \mathrm{mg}$ protein

$2 \mathrm{nmol} / \mathrm{mg}$ protein 
Figure 11 HPLC profile of OTA and its metabolites $4(\mathrm{R})$ - and 4(S)-4OH-OTA.

Typical HPLC profile of OTA metabolites fonned during incubation of OTA with liver microsomes from PCN treated rats. OTA metabolites wers: analysed as described in the Methods section 2.15.3. Extracted sample $(50 \mu \mathrm{L}$ of $0.5 \mathrm{~mL}$ ) was injected into a Perkin Elmer-Sıries 4 liquid chromatograph. The column used was Partisil 10 ODS-2. The solvent system consisted of a mixture of a) acctonitrile: methanol (500:500, v/v) $65 \%$ and b) $5 \mathrm{mM}$ sodium acetate:acetic acid $(500: 14, \mathrm{v} / \mathrm{v}) 35 \%$. The flow rate was $1.5 \mathrm{~mL} / \mathrm{min}$ and the fluorometric detection was performed at excitation wavelength of $340 \mathrm{~nm}$, emission wavelength of $465 \mathrm{~nm}$ and excitation/emission slits of $5 / 10 \mathrm{~nm}$. 


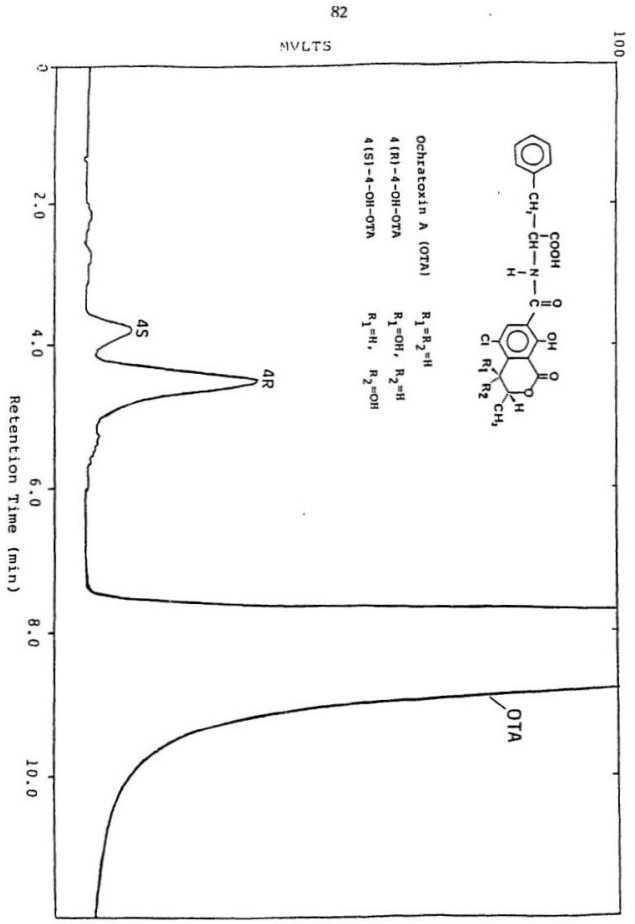


Table 11. Total cytochromes P-450 content of liver microsomes isolated from rats following various treatments.

Treatment*

Cyt. P-450 content

(nmol/mg protein)

Control

$0.85 \pm 0.07$

PB

$2.15 \pm 0.14^{*}$

3-MC

$1.70 \pm 0.18$ *

Clofibrate

$1.40 \pm 0.20$ *

Isosafrole

$1.00 \pm 0.02 *$

PCN

$1.45 \pm 0.17 *$

Isoniazid

$0.80 \pm 0.03$

* The various treatments are given in Table 7. Values shown are means \pm S.D. of duplicate determinations from four individual rats per treatment group. ${ }^{*}$, means significantly different from control ( $\mathrm{p}<0.05$, Mann Whitney test). 
Figure 12 The formation of $4(\mathrm{R})$ - and $4(\mathrm{~S}) \cdot 4 \cdot 0 \mathrm{H}-\mathrm{OTA}$ in vitro using liver microsomes from rats treated with different inducers of cytoch rome P.450.

Incubations were carried out in triplicate at $37^{\circ} \mathrm{C}$ for $30 \mathrm{~min}$ in $0.1 \mathrm{M}$ potassium phosphate buffer ( $\mathrm{pH}$ 7.4) and contained per mL: $2 \mathrm{mg}$ microsomal protein, $125 \mathrm{nmol}$ OTA and a NADPH-regenerating system (consisting of $0.4 \mu \mathrm{mol} \mathrm{NADP}+, 5 \mu \mathrm{mol} \mathrm{MgCl} 2,5 \mu \mathrm{mol} \mathrm{DL}$-isocitrate and 0.65 units of isocitric dehydrogenasc). Details are described in the Methods section 2.15 .3 for analysis of metabolites. Results are means \pm S.D. of four individual rats per treatment group. These results are from Batch III. Results from Batch II (a completely different batch of all treatments) are shown in figure 13. Batch I (not shown) was a trial run to determine the optimum doses of the various cytochrome P-450 inducers and their effects on OTA metabolism. *, means significantly different from control ( $\mathrm{p}<0.05$, Mann-Whitney test). 


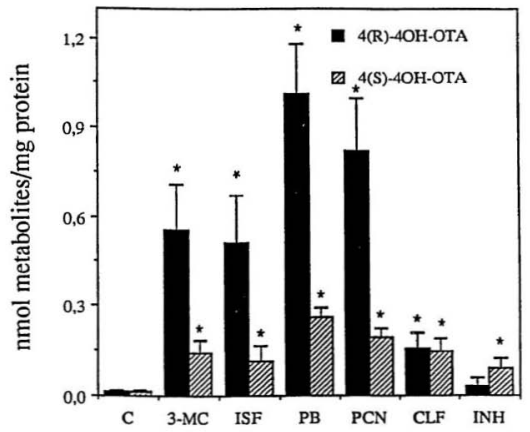


Figure 13 The formation of $4(\mathrm{R})$ - and $4(\mathrm{~S}) \cdot 4-0 H-O T A$ in vitro using liver microsomes from rats treated with different inducers of cytochrome P-450 (Batch II).

Incubations were carried out in triplicate at $37{ }^{\circ} \mathrm{C}$ for $30 \mathrm{~min}$ in $0.1 \mathrm{M}$ potassium phosphate buffer $(\mathrm{pH} 7.4)$ and contained per $\mathrm{mL}: 2 \mathrm{mg}$ microsomal protein, 125 nmol OTA and a NADPH-regenerating system (consisting of $0.4 \mu \mathrm{mol} \mathrm{NADP}+, 5 \mu \mathrm{mol} \mathrm{MgCl}, 5 \mu \mathrm{mol} \mathrm{DL}$-isocitrate and 0.65 units of isocitric dehydrogenase). Details are described in the Methods section 2.15.3 for analysis of metabolites. Results are means \pm S.D. of three individual rats per treatment group. 

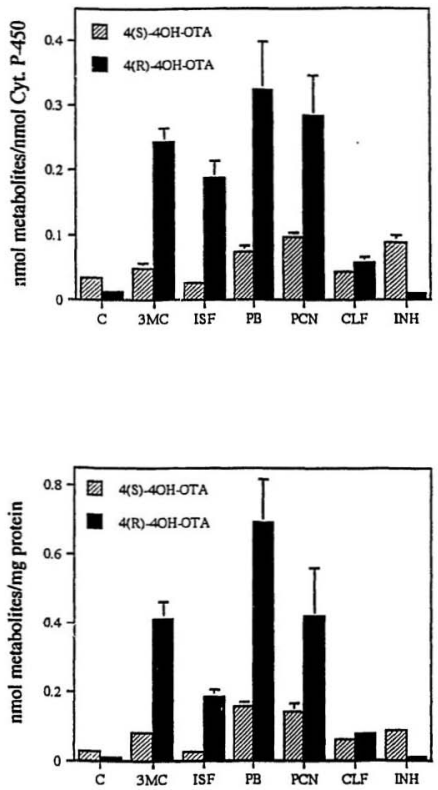
Figure 14 The formation of $4(\mathrm{R})$ - and $4(\mathrm{~S})-4-\mathrm{OH}-\mathrm{OTA}$ in vitro using liver microsomes from control rats treated with the vehicles of the different inducers of cytochrome P-450.

Incubations were carried out in triplicate at $37 \circ \mathrm{C}$ for $30 \mathrm{~min}$ in $0.1 \mathrm{M}$ potassium phosphate buffer ( $\mathrm{pH} 7.4$ ) and contained per $\mathrm{mL}: 2 \mathrm{mg}$ microsomal protcin, $125 \mathrm{nmol}$ OTA and a NADPH-regenerating system (consisting of $0.4 \mu \mathrm{mol} \mathrm{NADP}+5 \mu \mathrm{mol} \mathrm{MgCl}, 5 \mu \mathrm{mol} \mathrm{DL}$-isocitrate and 0.65 units of isocitric dehydrogenase). Details are described in the Methods section 2.15 .3 for analysis of metabolites. Results are means \pm S.D. of three individual rats per treatment group. Results are plotted to the same scale as the figure of the actual treatments (Figure 12 in the Results Section) for comparison. 


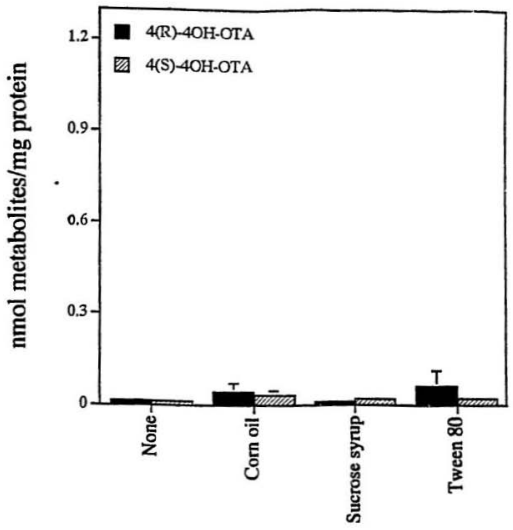


Table 12. Ethoxy- and pentoxyresorufin $\mathbf{O}$-dealkylation by liver microsomes from control, $3 \mathrm{MC}$ and $\mathrm{PB}$ treated rats.

Sample

PROD

EROD

(pmol resorufin released/min/mg protein)
Exp.1
Exp.2
Exp.1
Exp.2

$\begin{array}{lrrrr}\text { Control } & 17.8 & 19.4 & 22.4 & 19.0 \\ \text { 3MC } & 82.2 & 83.3 & 1840.0 & 1678.2 \\ \text { PB } & 543.6 & 594.7 & 234.1 & 379.7\end{array}$

Details are described in the Methods section 2.16. Results are means of triplicate incubations from two separate experiments. 
Figure 15 Effect of the cytochrome P-450 inhibitors $\alpha$-naphthoflavone $(50 \mu \mathrm{M})$ and metyrapone $(100 \mu \mathrm{M})$ on the metabolism of OTA to $4(\mathrm{R})-4-\mathrm{OH}-\mathrm{OTA}$ by liver microsomes from $\mathrm{PB}, 3 \mathrm{MC}$ and ISF treated rats.

Incubations were carried out in duplicate at $37^{\circ} \mathrm{C}$ for $20 \mathrm{~min}$ in $0.1 \mathrm{M}$ potassium phosphate buffer (pH 7.4) and contained per $\mathrm{mL}: 2 \mathrm{mg}$ microsomal protein, $125 \mathrm{nmol}$ OTA and a NADPH-regenerating system (consisting of $0.4 \mu \mathrm{mol} \mathrm{NADP}+, 5 \mu \mathrm{mol} \mathrm{MgCl}, 5 \mu \mathrm{mol} \mathrm{DL}$-isocitrate and 0.65 units of isocitric dehydrogenase). Details are described in the Methods section 2.15.3 for analysis of metabolites. Results are means of two separate experiments (A and B) from two different animals. The uninhibited activity was normalized to $100 \%$. The uninhibited values were $0.58 / 0.53$ for $\mathrm{PB}, 0.38 / 0.87$ for $3 \mathrm{MC}$ and $0.51 / 1$. 19 for ISF nmol $4(\mathrm{R})$ 4-OH-OTA/mg protein. 

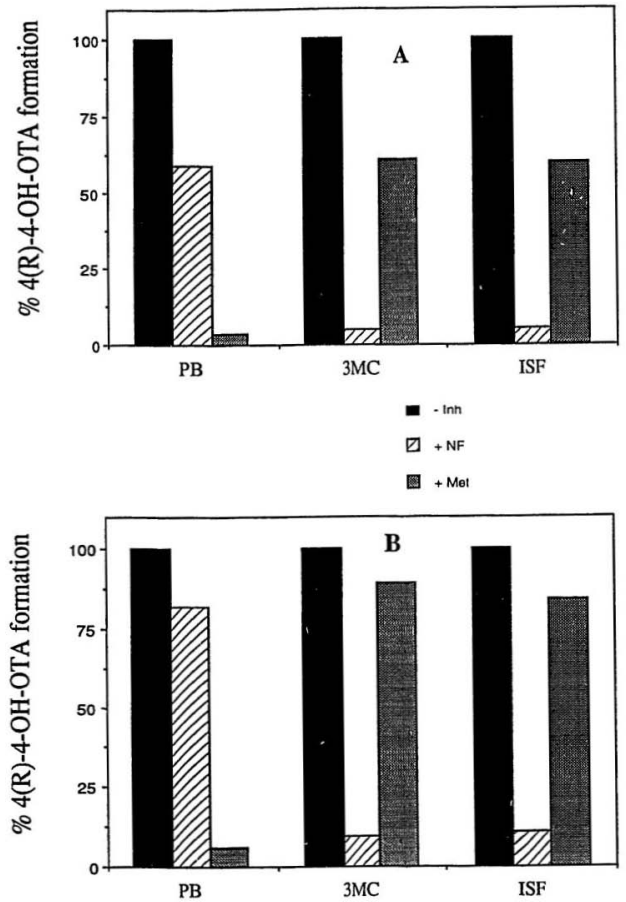
Figure 16 Effect of the cytochrome P.450 inhibitors \&-naphthoflavone $(50 \mu \mathrm{M})$ and metyrapone $(100 \mu \mathrm{M})$ on the metabolism of (OTA to $4(\mathrm{~S})-4-0 \mathrm{H}-\mathrm{O}$ TA by liver microsomes from $\mathrm{PB}, 3 \mathrm{MC}$ and ISF treated rats.

Incubations were carried out in duplicate at $370 \mathrm{C}$ for $20 \mathrm{~min}$ in $0.1 \mathrm{M}$ potassium phosphate buffer ( $\mathrm{pH} 7.4$ ) and contained per mL: $2 \mathrm{mg}$ microsomal protein, 125 nmol OTA and a NADPH-regenerating system (consisting of $0.4 \mu \mathrm{mol} \mathrm{NADP}+, 5 \mu \mathrm{mol} \mathrm{MgCl}, 5 \mu \mathrm{mol}$ DL-isocitrate and 0.65 units of isocitric dehydrogenase). Details are described in the Methods section 2.15.3 for analysis of metabolites. Results are means of two separate experiments (A and $B$ ) from two different animals. The uninhibited activity was normalized to $100 \%$. The uninhibited values were $0.18 / 0.20$ for PB, 0.13/0.14 for $3 \mathrm{MC}$ and $0.09 / 0.13$ for ISF nmol $4(\mathrm{R})$ 4-OH-OTA/mg protein. 

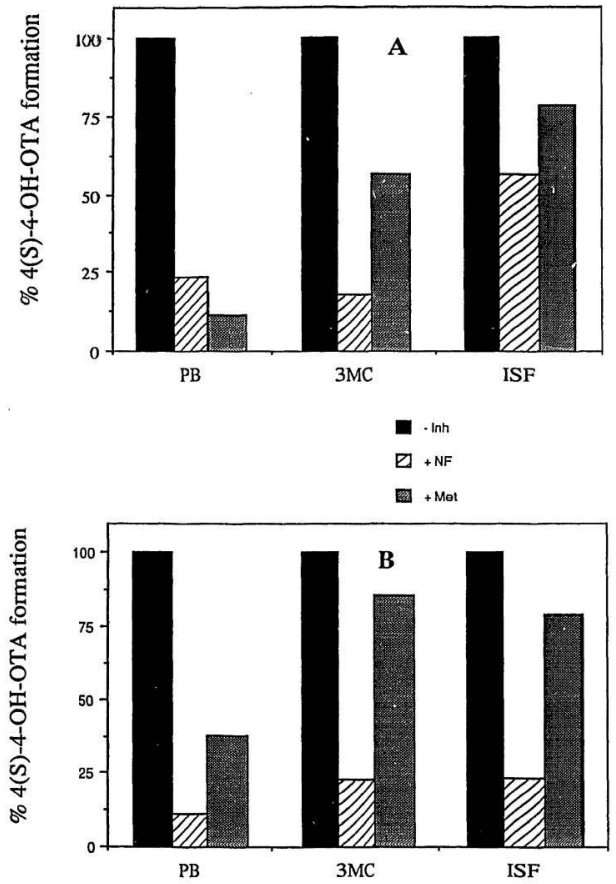
Figure 17a Effect of monoclonal antibody (MAb) 1-7-1 on the metabolism of OTA by microsumes from rats treated with $3 \mathrm{MC}$.

MAb was preincubated with microsomes in $0.1 \mathrm{M}$ potassium phosphate buffer ( $\mathrm{pH}$ 7.4) for $30 \mathrm{~min}$ at room temperature. Microsomal protein concentration was $2 \mathrm{mg} / \mathrm{mL}$ and the total incubation volume was $100 \mu \mathrm{L}$. Reaction was started by adding substrate (OTA, $125 \mu \mathrm{M}$ ) and NADPH. regenerating system and was carried out in duplicate at $370 \mathrm{C}$ for $30 \mathrm{~min}$.

Figure 17b Effect of rabbit IgG on the metabolism of OTA by microsomes from rats treated with $3 \mathrm{MC}$.

IgG was preincubated with microsomes in $0.1 \mathrm{M}$ potassium phosphate buffer ( $\mathrm{pH} 7.4$ ) for $30 \mathrm{~min}$ at room temperature. Microsomal protein concentration was $2 \mathrm{mg} / \mathrm{mL}$ and the total incubation volume was $100 \mu \mathrm{L}$. Reaction was started by adding substrate (OTA, $125 \mu \mathrm{M}$ ) and NADPH. regenerating system and was carried out in duplicate at $37^{\circ} \mathrm{C}$ for $30 \mathrm{~min}$. 

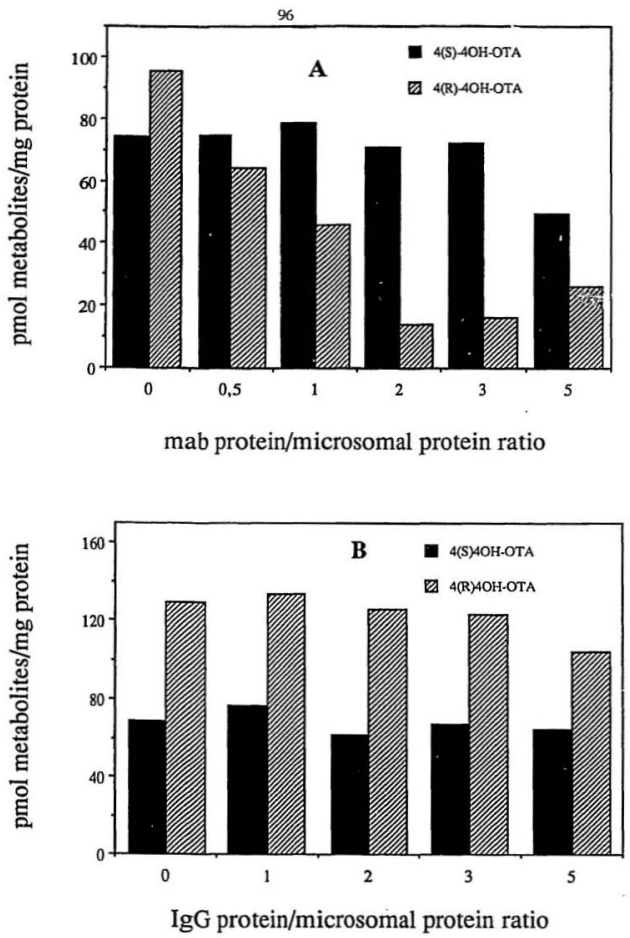
Figure 18a Effect of monoclonal antibody (MAb) 2-66-3 on the metabolism of OTA by microsomes from rats treated with PB.

MAb was preincubated with microsomes in $0.1 \mathrm{M}$ potassium phosphate buffer $(\mathrm{pH} 7.4)$ for $30 \mathrm{~min}$ at room temperature. Mierosomal protein concentration was $2 \mathrm{mg} / \mathrm{mL}$ and the total incubation volume was $100 \mu \mathrm{L}$. Reaction was started by adding substratc (OTA, $125 \mu \mathrm{M}$ ) and NADPHregenerating system and was carried out in duplicate at $37^{\circ} \mathrm{C}$ for $30 \mathrm{~min}$.

Figure 18b Effert of rabbit IgG on the metabolism of OTA by microsomes from $\mathrm{PB}$ treated rats.

IgG was preincubated with microsomes in $0.1 \mathrm{M}$ potassium phosphate buffer ( $\mathrm{pH}$ 7.4) for $30 \mathrm{~min}$ at room temperature. Microsomal protein concentration was $2 \mathrm{mg} / \mathrm{mL}$ and the total incubation volume was $100 \mu \mathrm{L}$. Reaction was started by adding substrate (OTA, $125 \mu \mathrm{M}$ ) and NADPHregenerating system and was carried out at $37^{\circ} \mathrm{C}$ for $30 \mathrm{~min}$. 


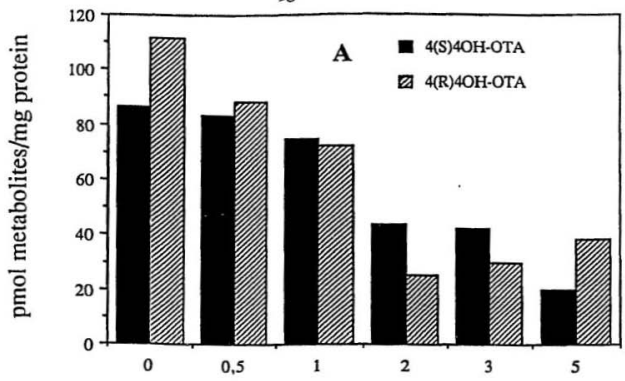

mab protein/microsomal protein ratio

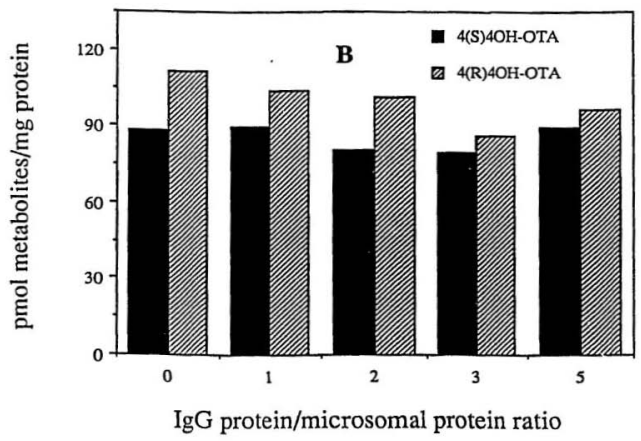


Table 13. Cross reactivity of MAb 1-7.1 and 2-66-3 with cytochromes P.450 in liver microsomes isolated from rats treated with $\mathrm{PB}$ or $3 \mathrm{MC}$.

\begin{tabular}{|c|c|c|}
\hline \multirow{3}{*}{ Treatment } & \multicolumn{2}{|c|}{ 4(R)-4-OH-OTA Inhibition (\%) } \\
\hline & & \\
\hline & $1.7 .1 *$ & $2.66 .3 *$ \\
\hline PB & 23.2 & 58.3 \\
\hline $3 \mathrm{MC}$ & 63.8 & 39.0 \\
\hline
\end{tabular}

4(S)-4-OH-OTA Inhibition (\%)

Treatment

MAb

\begin{tabular}{lll} 
& $1.7 .1^{*}$ & $2.66 \cdot 3^{*}$ \\
\hline PB & 19.2 & 38.0 \\
$3 \mathrm{MC}$ & 0.0 & 15.0
\end{tabular}

Conditions are as described in the legend of Figure $17 \mathrm{a}$.

* MAb protein/microsomal protein 2:1. 
Table 14. Effect of INH- and PCN-pretreatments on hepatic cytochrome P-450 levels and OTA metabolism.

Pretreatment

Cyt. P.450

4(R)-4-OH-OTA $\quad$ 4(S)-4-OH-OTA

nmollmg protein

$\begin{array}{llll}\text { None } & 0.85 \pm 0.07 & 0.015 \pm 0.004 & 0.014 \pm 0.006 \\ \text { INH } & 0.80 \pm 0.03 & 0.032 \pm 0.030(2) & 0.093 \pm 0.031(7)^{*} \\ \text { PCN } & 1.45 \pm 0.17 * & 0.816 \pm 0.150(55)^{*} & 0.194 \pm 0.040(14)^{*}\end{array}$

Pretreatment of rats and measurement of cytochrome P-450 levels and OTA metabolites was carried out as described in the Methods (sections 2.5.1, 2.9 and 2.15.3). Values shown are means \pm S.D. from four individual rats. The numbers in parenthesis are fold increase in OTA metabolites compared to no treatment.

*, means significantly different from no treatment $(\mathrm{p}<0.05$, Mann Whitney test). 
Figure 19 A. Time course of OTA metabolism by liver microsomes from INH-treated rats.

B. Time course of lipid peroxidation in liver microsomes from INH-treated rats.

Incubations were carried out at $370^{\circ} \mathrm{C}$ in $0.1 \mathrm{M}$ potassium phosphate buffer ( $\mathrm{pH} 7.4$ ) and contained per $\mathrm{mL}$ : microsomal protein $(2 \mathrm{mg}), 125 \mathrm{mmol}$ OTA and a NADPH-regenerating system (see Methods section 2.15.1). At timed intervals, two $0.5 \mathrm{~mL}$ samples were withdra vn, one for liPLC analysis of OTA metabolites, and the other for measurement of MDA levels. Details are described in the Methods sections 2.15.3 and 2.14.2. Results are means of duplicate determinations from each of two separate experiments. The difference between the two means at all time points was no more than $23 \%$ for metabolism and $16 \%$ for lipid peroxidation. The relatively high degree of variation is likely due to the cytochrome P-450 content of liver microsomes from every individual animal, also due to the fact that lipid peroxidation is a chain reaction and the rates of propigation are known to vary greatly from an experiment to another. 

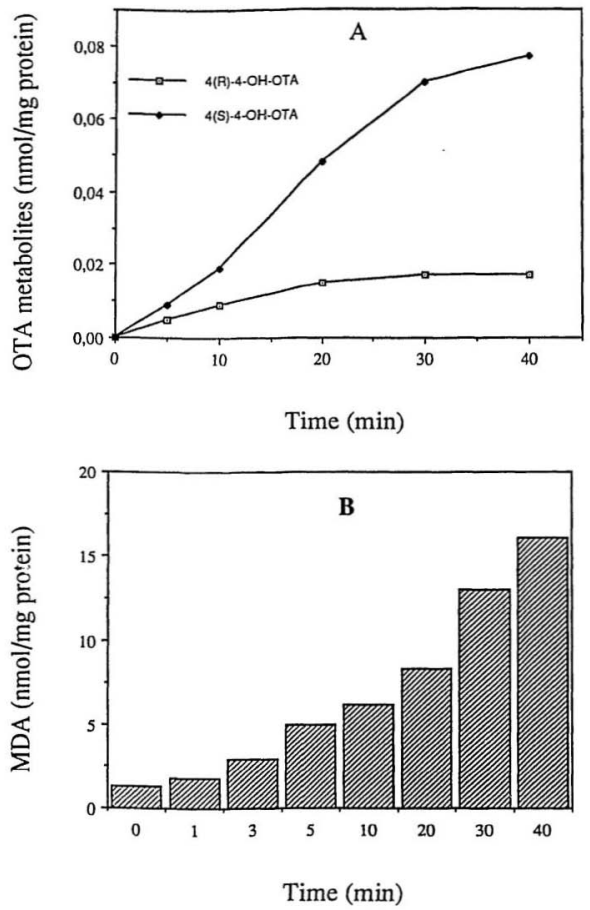
Figure 20 A. Tiine course of OTA metabolism by liver microsomes from PCN-treated rats.

B. Time course of lipid peroxidation in liver microsomes from PCN-treated rats.

Incubations were carried out at $37^{\circ} \mathrm{C}$ in $0.1 \mathrm{M}$ potassium phosphate buffer

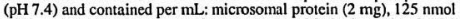
OTA and a NADPH-regenerating system (see Methods section 2.15,1). At timed intervals, two $0.5 \mathrm{~mL}$ samples were withdrawn, one for HPLC analysis of OTA metabolites, and the other for measurement of MDA levels. Details are described in the Methods sections 2,15.3 and 2.14.2. Results are means of duplicate determinations from ench of two separate experiments. The difference between the two means at all time points was no more than $18 \%$ for metabolism and $21 \%$ for lipid peroxidation. The relatively high degree of variation is likely due to the cy tochrome P-450 content of liver microsomes from every individual animal, also due to the fact that lipid peroxidation is a chain reaction and the rates of propagation are known to vary greatly from an experiment to another. 
104
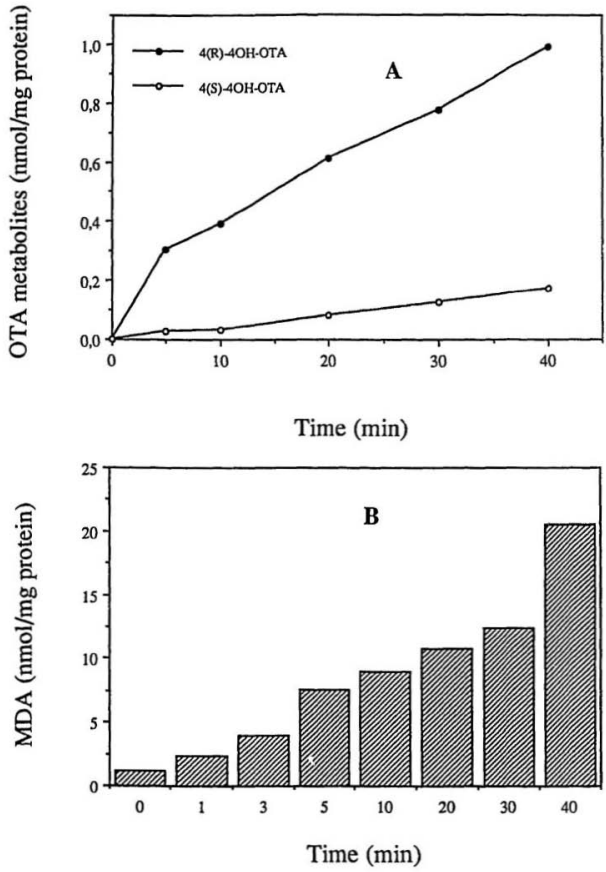
Figure 21 Effect of $\mathrm{pH}$ on OTA metabolism (A) and lipid peroxidation (B) by liver microsomes from INH-treated rats.

Incubations were carried out at $370^{\circ} \mathrm{C}$ in $0.1 \mathrm{M}$ potassium phosphate $(\mathrm{pH}$ $5.5-8.5)$ and contained in a total volume of $1 \mathrm{~mL}$ : microsomal protein (2 $\mathrm{mg}), 125$ nmol OTA and a NADPH-regenerating system (see Methods section 2.15.1). At the end of $30 \mathrm{~min}, 0.5 \mathrm{~mL}$ from each incubation was withdrawn for HPLC analysis of OTA metabolites, and the remaining 0.5 $\mathrm{mL}$ was used for measurement of MDA levels. Details are described in the Methods sections 2.15.3 and 2.14.2. Results are means of duplicate determinations from each of two separate experiments. The difference between the two means at all time points was no more than $20 \%$ for metabolism and $10 \%$ for lipid peroxidation. The relatively high degree of variation is likely due to the cytochrome P-450 content of liver microsomes from every individual animal. 
106
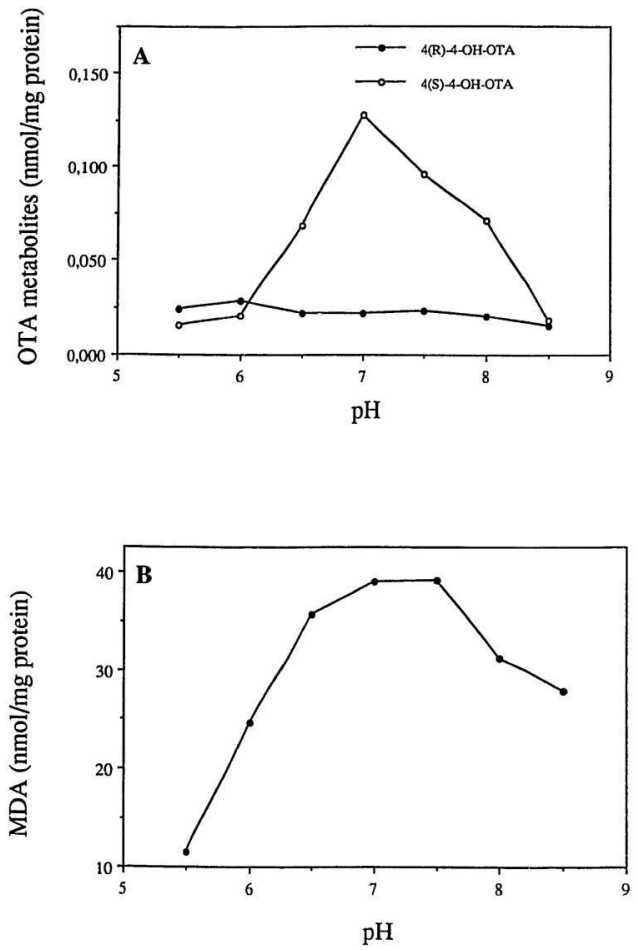
Figure 22 Effect of $\mathrm{pH}$ on OTA metabolism (A) and lipid peroxidation (B) by liver microsomes from PCN-treated rats.

Incubations were carried out at $370 \mathrm{C}$ in $0.1 \mathrm{M}$ potassium phosphate $(\mathrm{pH}$ $5.5-8.5)$ and contained in a total volume of $1 \mathrm{~mL}$ : microsomal protein (2 $\mathrm{mg}), 125 \mathrm{nmol}$ OTA and a NADPH-regenerating system (sec Methods section 2.15.1). At the end of $30 \mathrm{~min}, 0.5 \mathrm{~mL}$ from each incubation was withdrawn for HPLC analysis of OTA metabolites and the remaining 0.5 $\mathrm{mL}$ was used for measurement of MDA levels. Details are described in the Methods sections 2.15.3 and 2.14.2. Results are means of duplicate determinations from each of two separate experiments. The difference between the two means at all time points was no more than $40 \%$ for metabolism and $16 \%$ for lipid peroxidation. The high degree of variation is likely due to the cytochrome P-450 content of liver microsomes from every individual animal, also due to the fact that lipid peroxidation is a chain reaction and the rates of propagation are known to vary greatly from an experiment to another. 

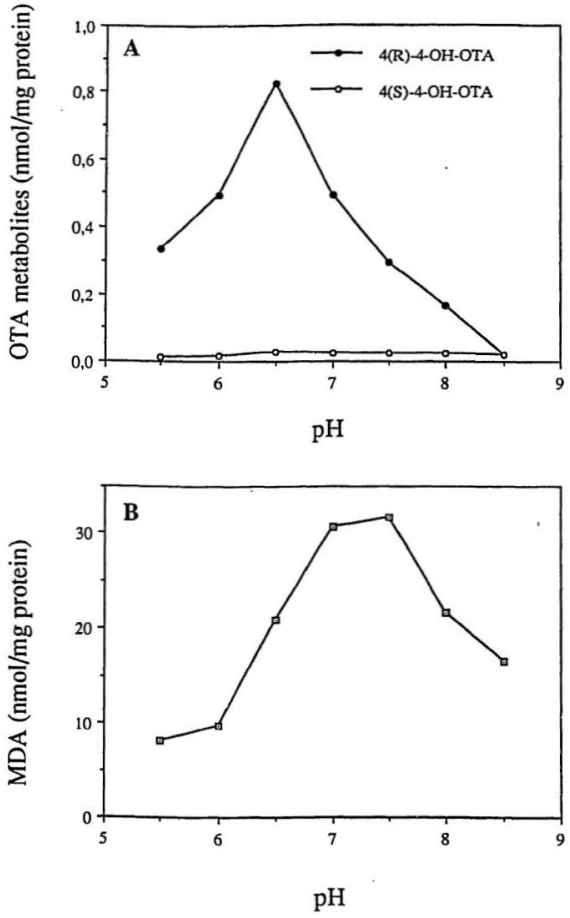
Table 15. Effect of active oxygen scavengers and antioxidant on NADPH-dependent OTA metabolism and lipid peroxidation.

\begin{tabular}{lccc}
\hline Addition & 4(R)-4-OH-OTA & 4(S)-4-OH-OTA & MDA \\
& & & \\
& Exp.1/ Exp.2 & Exp.1/ Exp.2 & Exp.1/ Exp.2 \\
\hline & & & \\
INH microsomes & & & \\
None & $0.012 / 0.016$ & $0.094 / 0.106$ & $26.4 / 26.9$ \\
SOD (35 units) & $0.012 / 0.018$ & $0.078 / 0.092$ & $26.2 / 27.5$ \\
Catalase (800 units) & $0.012 / 0.018$ & $0.079 / 0.093$ & $24.3 / 26.5$ \\
Mannitol (11 mM) & $0.012 / 0.016$ & $0.060 / 0.082$ & $22.7 / 22.9$
\end{tabular}

\section{PCN microsomes}

None

SOD (35 units)

Catalase ( 800 units)

Mannitol (11 mM)
$0.63 / 0.71$

$0.66 / 0.80$

$0.78 / 0.94$

$0.69 / 0.84$
$0.19 / 0.21$

$23.1 / 24.7$

$0.24 / 0.30$

$19.0 / 23.4$

$20.5 / 20.8$

$19.1 / 19.8$

Incubations were carried out for $\mathbf{3 0} \mathrm{min}$ as described in the Methods section 2.15.1. Results are means of duplicate determinations from each of two separate experiments. 
Table 16. Effect of antioxidants and iron chelators on OTA metabolism, lipid peroxidation and cytochrome P.450 content.

\begin{tabular}{|c|c|c|c|c|}
\hline \multirow[t]{2}{*}{ Addition } & \multirow[t]{2}{*}{ 4(R)-4-OH-OTA } & 4(S)-4-OH-OTA & MDA & \multirow[t]{2}{*}{ CytochromeP.450* } \\
\hline & & nmollmg prot & Exp1/Exp.2 & \\
\hline \multicolumn{5}{|l|}{ INH microsomes } \\
\hline None & $0.012 / 0.016$ & $0.094 / 0.106$ & $24.5 / 25.1$ & 0.49 (40 \% Loss) \\
\hline Desferal $(50 \mu \mathrm{M})$ & $0.025 / 0.031$ & $0.005 / 0.008$ & $1.15 / 1.31$ & 0.83 ( $0 \%$ Loss) \\
\hline BPS $(100 \mu \mathrm{M})$ & $0.019 / 0.025$ & $0.007 / 0.009$ & $0.81 / 0.89$ & \\
\hline BHA $(10 \mu M)$ & $0.011 / 0.015$ & $<0.001$ & $1.93 / 2.05$ & \\
\hline DPPD $(10 \mu \mathrm{M})$ & $0.005 / 0.011$ & $0.010 / 0.012$ & $0.68 / 0.72$ & \\
\hline \multicolumn{5}{|l|}{ PCN microsomes } \\
\hline None & $0.63 / 0.71$ & $0.19 / 0.21$ & $21.9 / 23.3$ & 0.33 (75 \% Loss) \\
\hline Desferal $(50 \mu \mathrm{M})$ & $1.32 / 1.54$ & $0.24 / 0.28$ & $1.20 / 1.64$ & 0.82 (37 \% Loss) \\
\hline BPS $(100 \mu \mathrm{M})$ & $1.74 / 1.86$ & $0.12 / 0.14$ & $1.00 / 1.10$ & \\
\hline BHA $(10 \mu \mathrm{M})$ & $0.65 / 0.69$ & $0.09 / 0.11$ & $1.90 / 2.4$ & \\
\hline DPPD $(10 \mu \mathrm{M})$ & $0.74 / 0.78$ & $0.09 / 0.11$ & $0.81 / 0.89$ & \\
\hline
\end{tabular}

Incubations were carried out for $30 \mathrm{~min}$ as described in the Methods section 2.15.1. Results are means of duplicate determinations from two separate experiments. * The original cytochrome P-450 contents were 0.83 and 1.32 $\mathrm{nmol} / \mathrm{mg}$ protein for microsomes isolated from rats treated with $\mathrm{INH}$ and $\mathrm{PCN}$ respectively. 
Figure 23 Effect of varying BHA concentrations on (A) OTA metabolism and (B) lipid peroxidation by liver microsomes from INH-treated rats.

Incubations were carried out at $37^{\circ} \mathrm{C}$ in $0.1 \mathrm{M}$ potassium phosphate $(\mathrm{pH}$ 7.4) for 10 and $30 \mathrm{~min}$ in the presence of $0-10 \mu \mathrm{M} \mathrm{BHA}$, and contained in a total volume of $1 \mathrm{~mL}$ : microsomal protein $(2 \mathrm{mg}), 125 \mathrm{nmol}$ OTA and a NADPH-regenerating system (see Methods section 2.15.1). At the end of $30 \mathrm{~min}, 0.5 \mathrm{~mL}$ from each incubation was withdrawn for HPLC analysis of OTA metabolites and the remaining $0.5 \mathrm{~mL}$ was used for measurement of MDA levels. Details are described in the Methods sections 2.15.3 and 2.14.2. Results are means of duplicate determinations from each of two separate experiments. The difference between the two means at all time points was no more than $33 \%$ for metabolism and $30 \%$ for lipid peroxidation. The relatively high degree of variation is likely due to the cytochrome P-450 content of liver microsomes from every individual animal, also due to the fact that lipid peroxidation is a chain reaction and the rates of propagation are known to vary greatly from an experiment to another. 


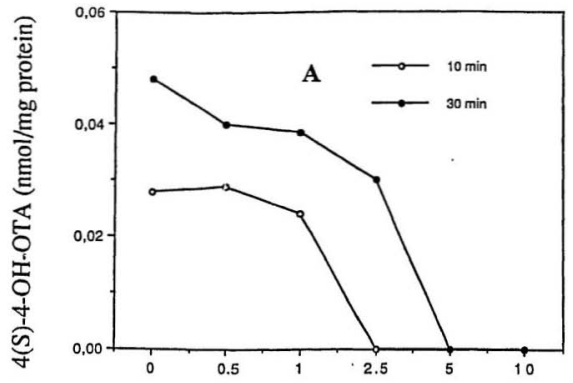

BHA ( $\mu \mathrm{M})$

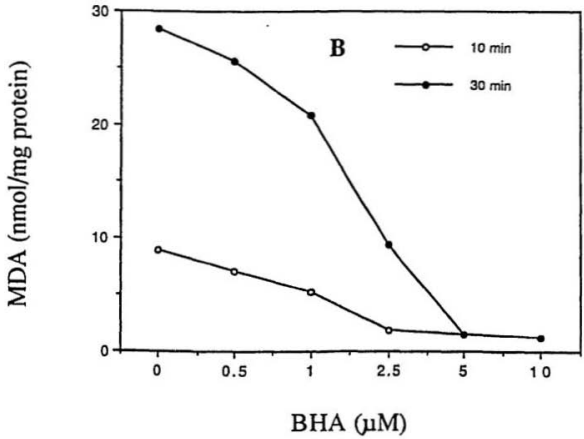


Table 17. Effect of BHA and mannitiol on ascorbate-dependent O'TA metabolism and lipid peroxidation.

Addition 4(R)-4-OH-OTA 4(S)-4-OH-OTA

MDA

nmollmg protein

Exp.1 / Exp.2 Exp.1/Exp.2 Exp.1 $/$ Exp.2

INH microsomes

None

$0.018 / 0.028$

$0.125 / 0.155$

$33.3 / 35.7$

Mannitol (11 mM)

$0.014 / 0.020$

$0.150 / 0.184$

$29.5 / 34.1$

$\mathrm{BHA}(10 \mu \mathrm{M})$

$0.014 / 0.021$

$0.012 / 0.028$

$2.50 / 2.96$

PCN microsomes

None

$0.010 / 0.050$

$0.129 / 0.137$

$28.2 / 33.4$

Mannitol (11 mM)

$0.021 / 0.037$

$0.122 / 0.138$

$26.1 / 29.0$

BHA (10 $\mu \mathrm{M})$

$0.020 / 0.026$

$0.008 / 0.016$

$0.77 / 0.81$

Incubations were carried out for $\mathbf{3 0} \mathrm{min}$ as described in the Methods section 2.15.1 with the exception that ascorbate/ $\mathrm{Fe}^{2}+(1 \mathrm{mM} / 5 \mu \mathrm{M}$, final concentrations $)$ were used instead of NADPH-regenerating system. Results are mean of duplicatc determinations from two separate experiments. 
Table18. Ability of various hemoproteins/hydroperoxides to metabolize OTA.

System

4(R)-4-OH-OTA

4(S)-4-OH-OTA

nmol /nmol heme

\begin{tabular}{lcc} 
PCN microsomes/CHP & $0.530 / 0.536$ & $0.017 / 0.039$ \\
$\mathrm{PCN}$ microsomes $/ \mathrm{H}_{2} \mathrm{O}_{2} \mathrm{a}$ & $0.014 / 0.016$ & $0.012 / 0.015$ \\
$\mathrm{PCN}$ microsomes/LAHP & $<0.001 \mathrm{~b}$ & $<0.001$ \\
$\mathrm{Hb} / \mathrm{CHP}$ & $<0.001$ & $<0.001$ \\
$\mathrm{Hb} / \mathrm{H}_{2} \mathrm{O}_{2}$ & $<0.001$ & $<0.001$ \\
$\mathrm{Hb} / \mathrm{LAHP}$ & $<0.001$ & $<0.001$ \\
$\mathrm{Hematin} / \mathrm{CHP}$ & $0.005 / 0.008$ & $0.007 / 0.015$ \\
$\mathrm{Hematin} / \mathrm{H}_{2} \mathrm{O}_{2}$ & $0.006 / 0.009$ & $0.002 / 0.004$ \\
Hematin/LAHP & $<0.001$ & $<0.001$ \\
\hline
\end{tabular}

Incubations were carried out for $30 \mathrm{~min}$ as described in the Methods section 2.15.1. For the different reagents, the following concentrations were used (in 0.5 $\mathrm{mL}$ reaction volume): $\mathrm{PCN}$ microsomes (1 $\mathrm{mg}$ protein), $\mathrm{Hb}(10 \mathrm{nmol})$, Hematin (2.5 nmol), CHP $(0.5 \mathrm{mM}), \mathrm{H}_{2} \mathrm{O}_{2}(5 \mathrm{mM})$, LAHP $(65 \mu \mathrm{M})$. Results are means of duplicate incubations from each of two separate experiments.

a $150 \mu \mathrm{M}$ hydroxylamine included to inhibit contaminating catalase.

b detection limit. 
Figure 24 Effect of $\mathrm{pH}$ on OTA metabolism (A), and lipid peroxidation (B) by liver microsomes from PB treated rats.

Incubations were carried out in duplicate at $370 \mathrm{C}$ for $30 \mathrm{~min}$ in $0.1 \mathrm{M}$ potassium phosphate buffer ( $\mathrm{pH} 5.5-8.5)$, and contained in a total volume of $1 \mathrm{~mL}$ : microsomal protein $(2 \mathrm{mg}), 125 \mathrm{nmol}$ OTA and a NADPH-regenerating system (see Methods section 2.15.1). At the end of $30 \mathrm{~min}, 0.5 \mathrm{~mL}$ from each incubation was withdrawn for HPLC analysis of OTA metabolites, and the remaining $0.5 \mathrm{~mL}$ was used for measurenient of MDA levels. Details for analysis of metabolites are described in the Methods section 2.15.3. Results are means of duplicate determinations from each of two separate experiments. The difference between the two means at all time points was no more than $17 \%$ for metabolism and $16 \%$ for lipid peroxidation. The relatively high degree of variation is likely due to the cytochrome P-450 content of liver microsomes from every individual animal, also due to the fact that lipid peroxidation is a chain reaction and the rates of propagation are known to vary greatly from an experiment to another. 

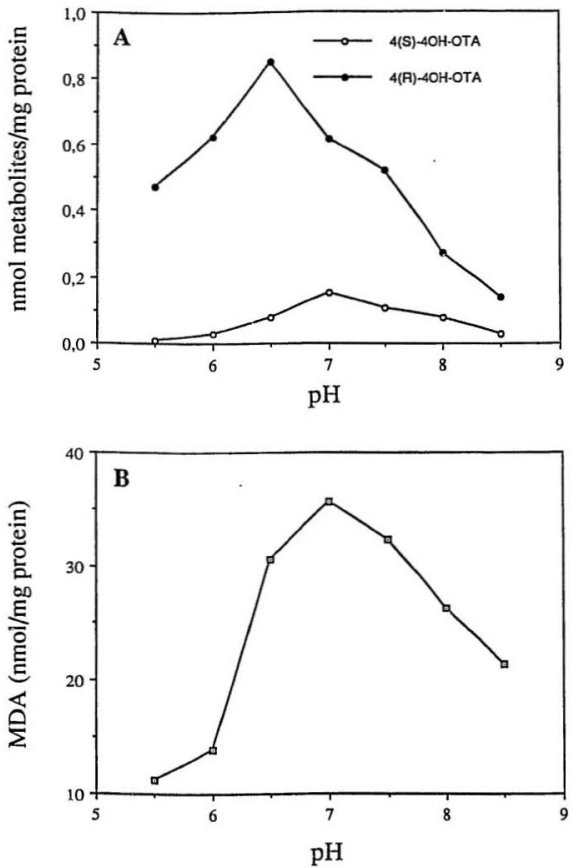
Figure 25 Effect of $\mathrm{pH}$ on OTA metabolism by liver microsomes from $3 \mathrm{MC}$ treated rats.

Incubations $\left(0.5 \mathrm{~mL}\right.$ volume) were carried out in duplicate at $37^{\circ} \mathrm{C}$ for 30 $\mathrm{min}$ in $0.1 \mathrm{M}$ potassium phosphate buffer ( $\mathrm{pH} 5.5-8.5$ ), and contained per $\mathrm{mL}$ : microsomal protein $(2 \mathrm{mg}), 125 \mathrm{nmol}$ OTA and a

NADPH-regenerating system (see Methods section 2.15.1). Details for analysis of metabolites are described in the Methods section 2.15.3.

Results are means of duplicate determinations from each of two separate experiments. The difference between the two means at all time points was no more than $37 \%$. The high degree of variation is likely due to the cytochrome P-450 content of liver microsomes from every individual animal. 


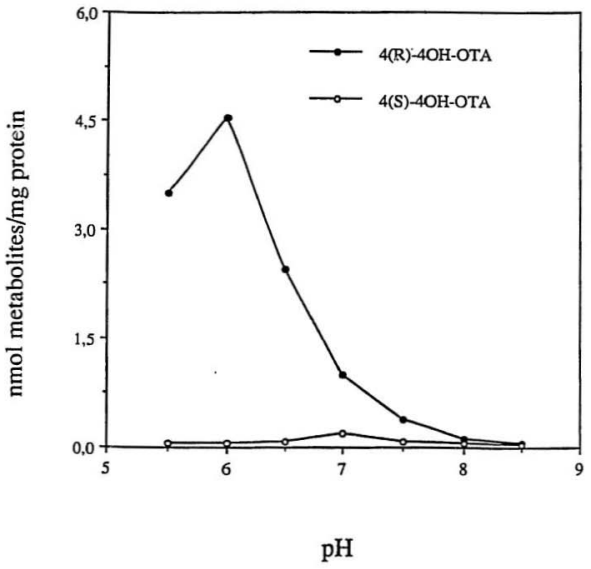


Table 19. Comparison of the ability of microsomes isolated from rats treated with $3 \mathrm{MC}$, ISF and PB to metabolize OTA at pH 6.0 and 7.4.

$\begin{array}{ccc}\text { Group } & \text { pH } & \text { 4(S)-4-OH-OTA } \\ (\mathrm{nmol} / \mathrm{mg} \text { protein) }\end{array}$

$\begin{array}{llll}\text { 3MC } & 6.0 & 0.057 \pm 0.030 & 7.98 \pm 3.10 \\ 3 \mathrm{MC} & 7.4 & 0.109 \pm 0.060 & 1.03 \pm 0.45 \\ \text { ISF } & 6.0 & 0.033 \pm 0.010 & 5.58 \pm 4.02 \\ \text { ISF } & 7.4 & 0.111 \pm 0.045 & 1.12 \pm 0.66 \\ \text { PB } & 6.0 & 0.042 \pm 0.033 & 0.63 \pm 0.21 \\ \text { PB } & 7.4 & 0.112 \pm 0.053 & 0.34 \pm 0.23\end{array}$

Details for conditions are described in the Methods section 2,15.1. Incubations were carried out in duplicate. Results are means \pm S.D. from three different animals. 
Table 20. The effect of $\mathrm{pH} 6.0$ and 7.4 on the ability of microsomes isolated from rats treated with $3 \mathrm{MC}, \mathrm{ISF}$ and $\mathrm{PB}$ to undergo lipid peroxidation and cytochrome P-450 destruction.

Treatment

$\mathrm{pH}$

MDA

Cyt. P-450 Loss (\%) (nmol/mg protein)

\begin{tabular}{lccc} 
3MC & 6.0 & $18.9 \pm 8.3$ & $31.6 \pm 7.0$ \\
3MC & 7.4 & $26.8 \pm 7.2$ & $57.3 \pm 3.5$ \\
ISF & 6.0 & $8.0 \pm 6.9$ & $14.7 \pm 20.5$ \\
ISF & 7.4 & $17.1 \pm 5.6$ & $42.6 \pm 20.8$ \\
PB & 6.0 & $11.8 \pm 8.8$ & $51.1 \pm 23.9$ \\
PB & 7.4 & $20.2 \pm 10.2$ & $59.2 \pm 25.4$ \\
\hline
\end{tabular}

Incubations were carried out at $370 \mathrm{C}$ for $30 \mathrm{~min}$ in $0.1 \mathrm{M}$ potassium phosphate buffer ( $\mathrm{pH}$ as indicated in the Table), and contained per $\mathrm{mL}: 2 \mathrm{mg}$ microsomal protein, $125 \mathrm{nmol}$ OTA and a NADPH-regenerating system (see Methods section $2,15.1$ ). The reaction volume was $3 \mathrm{~mL}$. At the end of $30 \mathrm{~min}, 0.5 \mathrm{~mL}$ was withdrawn for measurement of lipid peroxidation and the remaining volume was diluted 1:1 using the same incubation buffer $(\mathrm{pH} \mathrm{7.4)}$ to give $1 \mathrm{mg}$ microsomal protein/mL for measurement of cytochrome P-450 (see Methods section 2.9). Results are mean \pm S.D. from three different animals. 
Figure 26 Effect of $\mathrm{pH}$ on the $\mathrm{Km}$ and $\mathrm{Vmax}$ of OTA hydroxylation by liver microsomes from $3 \mathrm{MC}$ treated rats.

Incubations were carried out in duplicate at $37 \mathrm{GC}$ for 30 min in $0.1 \mathrm{M}$ potassium phosphate buffer (pH 6.0 or $\mathrm{pH} 7.4$ ), and contained per mL: microsomal protein $(2 \mathrm{mg})$, OTA $(10,25,50,75,125$ and $250 \mathrm{nmol})$ and a NADPH regenerating system (sce Methods section 2.15.1). The amounts of hydroxylated OTA in nmol/mg protein (the initial velocities) were measured at $30 \mathrm{~min}$ (the reaction was linear up to $60 \mathrm{~min}$ ). Only the major metabolite 4(R)-4-OH-OTA was considered in this case. Details for analysis of metabolites are described in the Methods section 2.15.3. Results are means of duplicate determinations from each of two scparate experiments. The difference between the two means at all time points was no more than $30 \%$. 

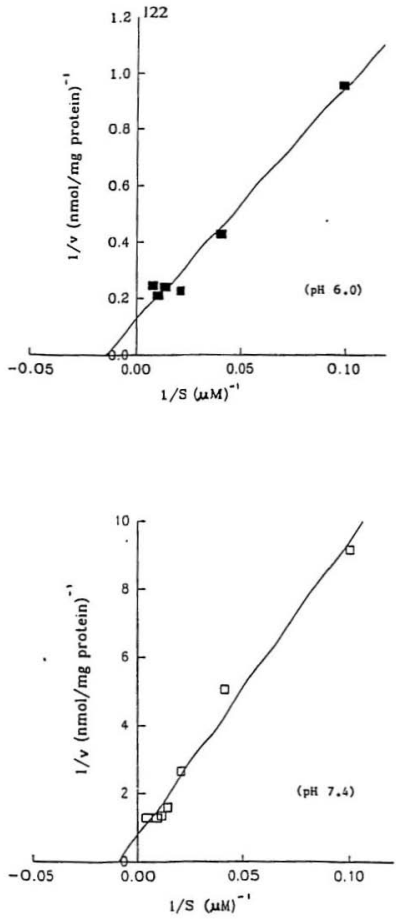
Figure 27 Effect of $\mathrm{pH}$ on the $\mathrm{Km}$ and $\mathrm{Vmax}$ of OTA hydroxylation by liver microsomes from ISF treated rats.

Incubations were carried out in duplicate at $377^{\circ} \mathrm{C}$ for $30 \mathrm{~min}$ in $0.1 \mathrm{M}$ potassium phosphate buffer ( $\mathrm{pH} 6.0$ or $\mathrm{pH} 7.4)$, and contained per $\mathrm{mL}$ : microsomal protein $(2 \mathrm{mg})$, OTA $(10,25,50,75,125$ and $250 \mathrm{nmol})$ and a NADPH-regenerating system (see Methods section 2.15.1). The amounts of hydroxylated OTA in $\mathrm{nmol} / \mathrm{mg}$ protein (the initial velocities) were measured at $30 \mathrm{~min}$ (the reaction was linear up to $60 \mathrm{~min}$ ). Only the major metabolite 4(R)-4-OH-OTA was considered in this case. Details for analysis of metabolites are described in the Methods section 2.15.3.

Results are means of duplicate determinations from each of two separate experiments. The difference between the two means at all time points was no more than $30 \%$. 

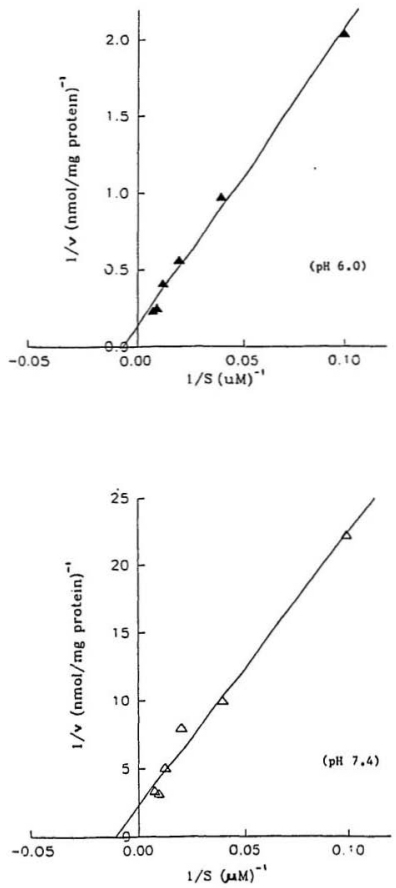
Figure 28 Alkaline phosphatase activity in urines of control and PBtreated rats following OTA administration.

Both groups of rats were given orally OTA $(0.5 \mathrm{mg} / \mathrm{kg}$ in $50 \mathrm{mM}$ $\mathrm{NaHCO}_{3}$ daily for 5 days). Details of pretreatments, treatments and enzyme measurements are given in the Methods sections 2.5.1, 2.6.1 and 2.18.1. Results are means \pm S.D. ( $n=8$ animals per treatment group). ${ }^{*}$, means significantly different from day 0 ( $p<0.05$, Mann-Whitney test). 


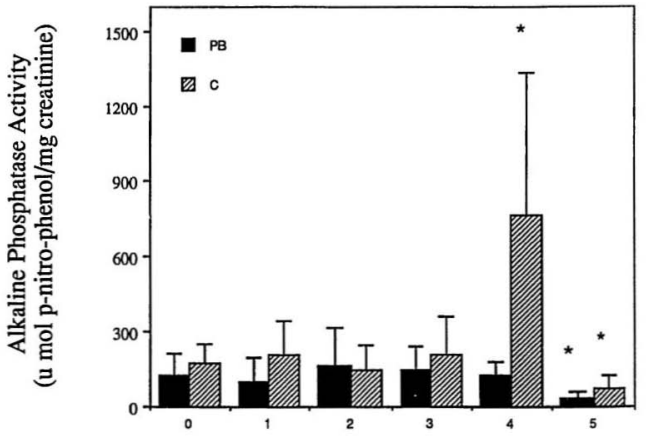

Day 
Figure 29 - -Glutamyl transferase activity in urines of control and PBtreated rats following OTA administration.

Both groups of rats were given orally OTA $(0.5 \mathrm{mg} / \mathrm{kg}$ in $50 \mathrm{mM}$ $\mathrm{NaHCO}_{3}$ daily for 5 days). Details of pretreatments, treatments and enzyme measurements are given in the Methods sections $2.5 .1,2.6 .1$ and 2.18.1. Results are means $\pm S . D$. $(n=8$ animals per treatment group). *, means significantly different form day 0 ( $p<0.05$, Mann-Whitney test). 


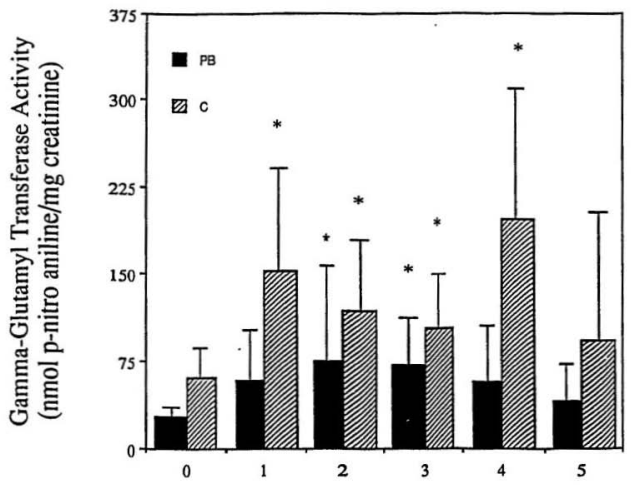

Day 
Figure 30 [14C]PAH transport in renal cortex slices from control (C), OTA treated (OTA) and PB-pretreated treated with OTA (PB + OTA) rats.

Rats were given orally OTA $(0.5 \mathrm{mg} / \mathrm{kg}$ in $50 \mathrm{mM} \mathrm{NaHCO} 3$ daily for 5 days. Details of pretreatments, treatments and PAH transport are given in the Methods sections 2.5.1, 2.6.1 and 2.17. Results are expressed as S/M (slice/medium) ratio at 10 and $30 \mathrm{~min}$ and represent means \pm S.D. (n $=4$ animals per tretment group). ${ }^{*}$, means significantly different from control $(p<0.05$, Mann-Whitney test). 
130

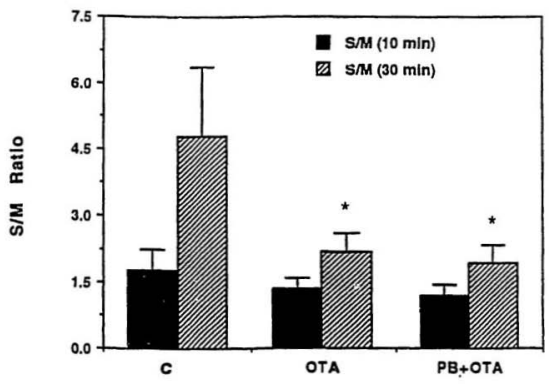


Figure 31 [3H]OTA levels in serum and urine of control and PB-treated rats 6 hours after one oral treatment with [3II)TA (288 $\mu \mathrm{g} / \mathrm{kg}$ in $50 \mathrm{mM} \mathrm{Nc:IC \textrm {C } _ { 3 }}$ ).

Details for treatments, sample processing and radioactivity counting are given in the Methods sections 2.6.2 and 2.18.2. Results are means \pm S.D. $\left(n=4\right.$ animals per treatment group). ${ }^{*}$, means significantly different from control $(p<0.05$, Mann-Whitncy test). 


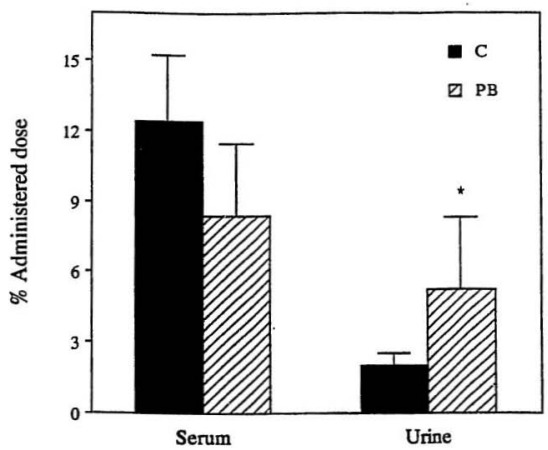


Figure 32 [3H]OTA levels in liver and kidney of control and PB-treated rats 6 hours after one oral treatment with [3H]OTA (288 $\mu \mathrm{g} / \mathrm{kg}$ in $50 \mathrm{mM} \mathrm{NaHCO}$ ).

Details for treatments, sample processing and radioactivity counting are given in the Methods sections 2.6.2 and 2.18.2. Results are menns \pm S.D. ( $n=4$ animals per treatment group). 


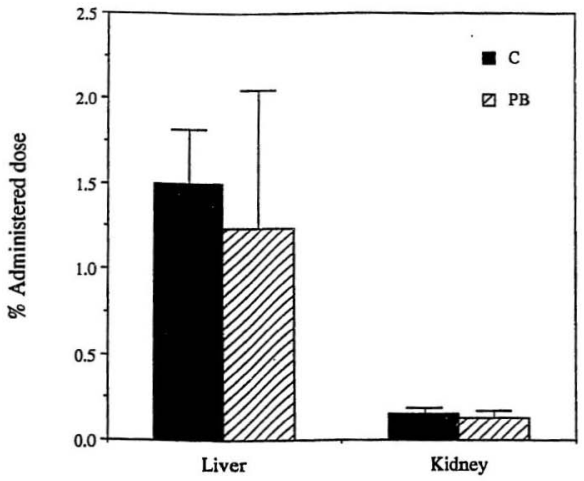


Figure 33 [3H]OTA levels in the gut of control and PB-treated rats 6 hours after one oral treatment with [3H]OTA $(288 \mu \mathrm{g} / \mathrm{kg}$ in 50 $\mathrm{mM} \mathrm{NaHCO}_{3}$ ).

Details for treatments, sample precessing and radioaclivity counting are given in the Methods sections 2.6.2 and 2.18.2. Results are means \pm S.D. ( $n=4$ animals per treatment group).

*, means $\mathrm{C}$ and $\mathrm{PB}$ are significantly different from each other $(\mathrm{p}<0.05$. Mann-Whitney test). 


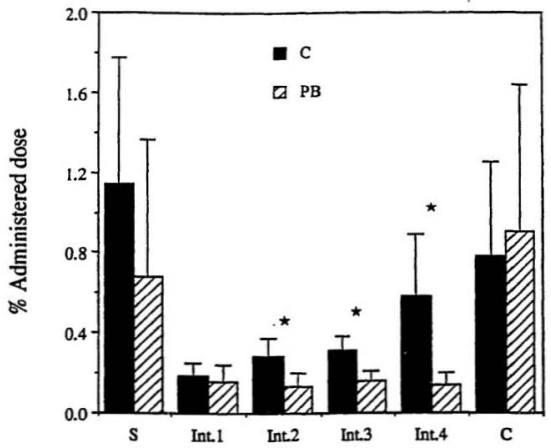

Gut segment 
Figure 34 [3H]OTA levels in the gut contents of control and PB-treated rats 6 hours after one oral treatment with [3II]OTA (288 $\mu \mathrm{g} / \mathrm{kg}$ in $50 \mathrm{mM} \mathrm{NaHCO}$ ).

Details for treatments, sample precessing and radioactivity counting are given in the Methods sections 2.6.2 and 2.18.2. Results are means \pm S.D. ( $n=4$ animals per treatment group).

$*$, means $C$ and $P B$ are significantly different from ench other $(p<0.05$, Mann-Whitney test). 


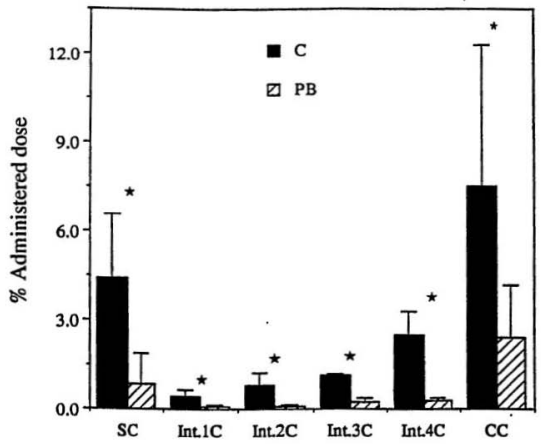

Gut contents 


\section{CHAPTER 4}

\section{DISCUSSION}

\subsection{Involvement of cytochrome P-450 in the stimulation of ochratoxin A- induced lipid peroxidation.}

Recently, using a reconstituted microsomal lipid peroxidation system consisting of microsomal phospholipid, the flavoprotein NADPH cytochrome P-450 reductase (Fp), EDTA and $\mathrm{Fe}^{3+}$, we demonstrated that OTA induced lipid peroxidation by chelating $\mathrm{Fe}^{3+}$ and that the resulting OTA-Fe $3+$ chelate was readily reducible by the flavoprotein NADPH cytochrome P-450 reductase to the OTA-Fe'2+ complex. The latter, in the presence of oxygen, provided the active species that initiated lipid peroxidation (Omar et al., 1990). In our reconstituted system, the addition of $25 \mu \mathrm{M}$ EDTA gave a maximum stimulation (4,0fold at $20 \mathrm{~min}$ ) in lipid peroxidation. Pederson and Aust (1972) first developed a reconstituted lipid peroxidation system consisting of phospholipid vesicles, Fp, NADPII, and iron chelates. In their system, an EDTA-Fe ${ }^{3+}$ chelate was required in addition to the standard ADP-Fe ${ }^{3+}$ chelate. Microsomal lipid peroxidation was first described by Hochstein and Ernster (1963), who demonstrated the enzymatic nature of the process and a requirement for NADPH and an ADP-Fe3+ chelate (Hochstein et al., 1964). The observation that only ADP-Fe ${ }^{3+}$ is necessary to promote lipid peroxidation in microsomes, while both EDTA-Fe ${ }^{3+}$ and ADP-Fe ${ }^{3+}$ are needed to promote it in a reconstituted system, suggests that there may be microsomal component(s) that directly reduce $\mathrm{AD}^{\prime}$ - $-\mathrm{Fe}^{3}+$ for which EDTA-Fe ${ }^{3+}$ can substitute in the reconstituted system (Morchousc and Aust, 1988). Cytochrome P-450, being the last electron acceptor among the catalytic components of the NADPH-dependent electron transport chain, is a likely candidate. Ekstrom and InglemanSundberg (1984) were the first to show that incorporation of one of several purified rabbit liver cy tochrome $\mathrm{P}-450$ isozymes into phospholipid vesicles containing Fp increased lipid 
peroxidation. Later, Morehouse and Aust (1988) demonstrated that purified rat liver cytochrome P-450b could effectively replace EDTA-Fe3+ in a reconstituted lipid peroxidation system consisting of phospholipid vesicles, EDTA-Fe ${ }^{3+}, \mathrm{ADP}-\mathrm{Fe}^{3+}, \mathrm{Fp}$, and NADPH. Our results show that cytochrome P-450 IIB 1 is also able to enhance lipid peroxidation in a reconstituted system where OTA- $\mathrm{Fe}^{3+}$ is the chelated form of $\mathrm{Fe}^{3+}$. That cytochrome P-450 plays a functional role, and is not merely a convenient source of hemeiron, is evident from the observation that heat-denatured cytochrome P-450, hematin, or a variety of hemoproteins were either ineffective or only marginally effective in stimulating lipid peroxidation (Table 9). Even cytochrome $c$, a hemoprotein readily reducible by NADPH-cytochrome P-450 reductase, only weakly stimulated lipid peroxidation. Carbon monoxide (CO) did not inhibit lipid peroxidation. We are unaware of any work in the literature showing the effects of $\mathrm{CO}$ on lipid peroxidation in a reconstituted mixed-function oxidase system. However, Ernster and Nordenbrand (1982) showed that CO did not inhibit the NADPH-dependent ADP/Fe ${ }^{3+-s t i m u l a t e d ~ l i p i d ~ p e r o x i d a t i o n ~ o f ~ l i v e r ~}$ microsomes. The reason for this lack of inhibition by $\mathrm{CO}$ is not clear.

The role of cytochrome P-450 in OTA-stimulated NADPH-dependent microsomal lipid peroxidation is also implicated from the data showing that microsomes isolated from Co-heme treated rats underwent less lipid peroxidation than those from control rats (Figure 10). Co-heme treatment has been shown to reduce both cytochrome P-450 and Fp levels, and thus the reduced ability of $\mathrm{Co}$-heme microsomes to peroxidize might be due to decreased cytochrome P-450 and/or Fp levels. It is well known that the activity of $\mathrm{Fp}$ greatly exceeds the overall mixed-function oxidase activity of the various microsomal cytochrome P-450-dependent mixed-function oxidases (Spaethe and Jollow, 1989). Thus, it seems unlikely that the decrease in Fp activity observed (Table 10) would play a significant role in the depression of lipid peroxidation. In support of this assertion, we 
have observed that fortification* of Co-heme microsomes with Fp (to levels $78 \%$ of that in control microsomes; Table 10) only partially increased the extent of lipid peroxidation to $45 \%$ of that of control microsomes (Figure 10). This suggests that lower cytochrome P. 450 levels rather than lower Fp levels were the main cause of decreased lipid peroxidation in Co-heme microsomes. However, it is possible that externally added Fp is not well integrated into the microsomes and thus may not be able to function as effectively as the Fp in control microsomes.

* Co-heme microsomes ( $2 \mathrm{mg}$ protein) were preincubated with X4 Fp (640 nmol) which was subsequently removed by centrifugation, leaving only $\mathrm{Fp}$ actually incorporated into the membrane or adhering to it. 


\subsection{Role of cytochrome P.450 in OTA metabolism.}

Stormer and Pederson (1980) were the first to show that OTA was metabolized to a hydroxylated product (4-OH-OTA) by rat liver microsomes. Later, they (Stormer et al., 1981) showed the formation of both $4(\mathrm{R})$ - and 4(S)-4-OH-OTA by human liver microsomes as well as liver microsomes from control rats and pigs. A subsequent study by Stormer et al. (1983) examined the metabolism of OTA by liver microsomes from control and PB treated rabbits, and Ueno (1985b) also used $3 \mathrm{MC}$ and $\mathrm{PB}$ as specific inducers of cytochrome P-450 enzymes in a study of OTA metabolism. Recently, Oster et al. (1991) showed that isolated cytochrome P-450 fractions (not identified) were able to metabolize OTA. Hietanen et al. (1991) showed that a cytochrome P-450 isozyme similar to that induced by $3 \mathrm{MC}$ is responsible for OTA metabolism. None of the above studies were of sufficient detail to examine under the same experimental conditions the effect of several major cytochrome P-450 inducers on OTA metabolism. We, therefore, carried out a detailed study of OTA metabolism by liver microsomes from rats treated with inducers of the major cytochrome P-450 isoforms.

We examined the OTA metabolizing ability of liver microsomes from rats pretreated with the cytochrome P-450 inducers phenobarbital (PB), 3-methylcholanthrene (3MC), clofibrate (CLF), isosafrole (ISF), pregnenolone-16*-arbonitrile (PCN) and isoniazid (INH). Proper controls for the vehicles in which inducers were given were done in addition to a no treatment control group. The microsomal cytochrome P-450 contents of livers from the different treatment groups were in good agreement with reported values (Gucngerich et al., 1982b; Hietanen et al., 1986; Graves et al., 1987). For the formation of the major metabolite 4(R)-4-OH-OTA, liver microsomes from rats treated with PB, PCN, 3MC, ISF and CLF gave 67, 55, 39, 34 and 10 fold increase respectively as compared to control microsomes from untreated rats. For 4(S)-4-OH-OTA (minor metrbolite) formation, liver microsomes from rats treated with $\mathrm{PCN}, \mathrm{PB}, 3 \mathrm{MC}, \mathrm{CLF}$ and 
ISF gave a 14, 18, 10, 10 and 8 fold increase compared to control microsomes from untreated rats (Figure 12). Microsomes from INH-treated rats did not give any increase in the formation of the $4(R)$ isomer, but formation of the $4(S)$ isomer was increased about 7 fold as compared to microsomes from control rats (no treatment) (Figure 12). The large increases in OTA metabolism by microsomes from 3MC- and PCN-treated animals were not due to the vehicles (corn oil and Tween 80 , respectively) in which they were administered. Both corn oil and Tween 80 did, however, increase the $4(R)$ and the $4(S)$ isomers formation over untreated controls by $2.9 / 2.4$-fold and $4.3 / 1$.6-fold respectively. Sucrose syrup, the vehicle in which clofibrate was administered, did not increase OTA metabolism (Figure 14).

OTA hydroxylation activity as well as its inducibility appears to vary with the strain of rat used. Hietanen et al. (1986) observed OTA 4-hydroxylation rates of about 2.5 and about $0.7 \mathrm{pmol} / \mathrm{min} / \mathrm{mg}$ protein with liver microsomes from female Lewis and DA rats respectively. This activity was inducible, about 2.3 -fold and about 1.3 -fold in Lewis rats, and about 12-fold and about 2-fold in DA rats after 3MC and PB pretreatments, respectively. On the other hand, Stormer and Pedersen (1980) found a very high rate of OTA 4-hydroxylation (about $90 \mathrm{pmol} / \mathrm{min} / \mathrm{mg}$ protein) in liver microsomes from male Wistar rats, and this activity was inducible 3.4-fold on PB pretreatment. In all the above studies, it was not indicated whether just 4(R)-4-OH-OTA (the major metabolite) or both epimers were measured. By comparison, we observed OTA 4-hydroxylation rate of about $1 \mathrm{pmol} / \mathrm{min} / \mathrm{mg}$ protein [Table 14; combined $4(\mathrm{R})$ and $4(\mathrm{~S})$ metabolites] which is morc in line with the values observed by Hietanen et al. (1986). This activity was inducible about 3.8 fold on INH pretreatment but 33 fold on PCN pretreatment (Table 14, values given in text are for combined metabolites). However, the inability of INH pretreatment to induce 4(R)-4-OH-OTA formation suggests that cytochrome P-450 HE1, the major isoform induced by INH (Ryan et al., 1985) is unable tc catalyze its formation. The effect of INH 
and PCN pretreatments on OTA metabolism has not previously been examined.

Cytochrome P-450 enzymes range from being very specific to non-specific towards substrates. A few substrates are known to react specifically with certain isoforms of cytochrome P-450 e.g. 7-pentoxyresorufin and 7-ethoxyresorufin are known to be specific substrates for cytochromes P-450 IIB1 (PB inducible) and 1AI (3MC inducible) respectively (Lubet et al., 1985; Burke and Mayer, 1983). We used these two substrates to confirm the induction of the desired cytochrome $\mathrm{P}-450$ isoforms by the inducers. Thus, microsomes from PB treated rats showed a 30 -fold increase in 7-pentoxyresorufin metabolism over control microsomes while microsomes from 3MC treated rats showed only a 4.5-fold increase. In contrast, microsomes from $3 \mathrm{MC}$ treated animals were 82 / 88 fold more effective than control microsomes in metabolizing 7-ethoxyresorufin while microsomes from PB treated animals were only 10.5 / 20-fold more efficient (Table 12). These results confirm that $\mathrm{PB}$ and $3 \mathrm{MC}$ treatments preferentially induced cytochromes $\mathrm{P}$. 450 IIB1 and LA1/LA2 respectively.

Cytochrome P-450 inhibitors are also useful tools in characterizing the involvement of specific cytochrome P-450 isoforms in metabolic processes. Some inhibitors react specifically with certain isozymes. For example, $\alpha$-naphthoflavone (Wiebel et al., 1971) and metyrapone (Jonen et al., 1974) are two inhibitors of cytochromes P-450 IA1/LA2 and IIB 1 respectively. Inhibition by specific cytochrome $\mathrm{P}-450$ inhibitors of a certain reaction that proceeds via a specific cytochrome $\mathrm{P}-450$ isozyme would mean the direct involvement of that particular isozyme in the reaction. $\alpha$-Naphthoflavone and metyrapone were used in our studies. $\alpha$-Naphthoflavone selectively inhibited $(89 / 95 \%)$ formation of $4(\mathrm{R})-4-\mathrm{OH}-$ OTA by microsomes from $3 \mathrm{MC}$ and ISF treated rats, but it inhibited its formation by microsomes from PB treated rats by only $18 / 41 \%$ (Figure 15). On the other hand, metyrapone inhibited $(94 / 96 \%)$ formation of the $4(R)$ isomer by microsomes from PB treated rats, but only inhibited its formation in microsomes from rats treated with $3 \mathrm{MC}$ and 
ISF (40/11\% and $40 / 16 \%$ ) (Figure 15). The effect of the two inhibitors on $4(\mathrm{~S})-4 \mathrm{OH}$ OTA formation was less selective (Figure 16). Liver microsomes from rats pretreated with Cobalt protoporphyrin LX (total cytochrome P-450 depleted by $>80 \%$ ) failed to metubolize OTA indicating the necessity for cytochrome P-450 (data not shown).

Use of monoclonal antibodies (MAbs) against specific isoforms of cytochrome P450 also provides additional evidence for the involvement of these isoforms in a reaction. Clones 1-7-1 (against cyrochrome P-450 IA 1,/A2) and 2-66-3 (against cytochrome P-450 IIB1) (Nakajima et al, 1990; Gelboin, personal communication) were used in these studies. The highest inhibition of OTA hydroxylation was given by only a two fold ratio of MAb protein/ microsomal protein using MAb from clone 1-7-1 with microsomes from 3MC treated rats (Figure 17a) and using MAb from clone 2-66-3 with microsomes from PB treated rats (Figure 18a). In both cases, a dose dependent inhibition was observed. Small non-specific reaction (inhibition of up to $22 \%$ ) was observed with the two microsomes when rabbit IgG was used instead of the MAbs (Figures 17b and 18b). The specificity of MAbs differ with substrates. A given MAb may inhibit very specifically a reaction of a certain substrate, but show less or even no specificity towards another substrate; this can be seen from the results obtained by Gelboin et al. (1988). From my results, MAb from clone 2-66-3 inhibited more strongly ( $58 \%$ inhibition) OTA metabolism $104(\mathrm{R})-4 \mathrm{OH}-\mathrm{OTA}$ by microsomes from PB treated rats although it cross reacted substintially (39\% inhibition) with microsomes from $3 \mathrm{MC}$ treated rats. On the other hand, $\mathrm{MAb}$ from clone 1-7-1 was more specific ( $64 \%$ inhibition) for microsomes from $3 \mathrm{MC}$ treated rats although it cross reacted weakly (23\% inhibition) with microsomes from PB treated rats (Table 13). 


\subsection{Possible mechanism of the formation of $4(\mathrm{~S})-4-\mathrm{OH}-\mathrm{OTA}$.}

Microsomes from INH treated rats [which mainly form the $4(\mathrm{~S})$ isomer] were chosen to investigate the mechanism of the formation of the $4(\mathrm{~S})$ isomer. Microsomes from PCN treated rats [that give a high yield of the $4(R)$ isomer] were chosen to study its formation. PCN pretreatment increased the formation of 4(R)-4-OH-OTA more than 50 -fold that in controls. In contrast, INH pretreatment did not substantially increase formation of the $4(R)$ isomer, but increased levels of the $4(S)$ isomer 7 -fold over control (Table 14).

The formation of 4(R)-4-OH-OTA and 4(S)-4-OH-OTA showed clear differences with respect to $\mathrm{pH}$ optima, effect of iron chelator and antioxidants. The $\mathrm{pH}$ optima for the formation of the $4(\mathrm{R})$ isomer by microsomes from rats treated with INH and PCN were pH 6.0 and 6.5 respectively (Figures 21 and 22). At $\mathrm{pH} 7.5$ there was a $60 \%$ decrease in the formation of the $4(\mathrm{R})$ isomer by microsomes from rats treated with PCN (Figure 22). A $\mathrm{pH}$ optimum of 6.5 for the fomation of the $4(\mathrm{R})$ isomer was also observeci for microsomes from rats treated with PB (Figure 24a), and of 6.0 for microsomes from rats treated with $3 \mathrm{MC}$ (Figure 25). Microsomes from ISF treated rats also showed a $\mathrm{pH}$ optimum of 6.0 with respect to the $4(\mathrm{R})$ isomer formation (data not shown). The $\mathrm{pH}$ optimum for cytochrome P-450-dependent oxidation of most substrates is around 7.5 so it is curious that the formation of the $4(\mathrm{R})$ isomer exhibits a low $\mathrm{pH}$ optimum. The phenolic hydroxyl of OTA has a pKa of 7.1 (Chu, 1971) so it is tempting to speculate that OTA with an unionized hydroxyl group binds preferentially to cytochrome $\mathrm{P}-450$ thus possibly yielding more 4(R)-4-OH-OTA below this $\mathrm{pH}$. The $\mathrm{pH}$ optimum of 6.5 for the formation of the 4(R) isomer differs from the value of 7.5 observed by Stormer and Pedersen (1980) with liver microsomes from phenobarbital treated Wistar rats, but agrees with the optimum $\mathrm{pH}$ obtained by Ueno (1985b) with liver microsomes from rats (strain not mentioned). 
In contrast to 4(R)-4-OH-OTA, the $\mathrm{pH}$ optimum of 7.0 observed for the formation of 4(S)-4-OH-OTA with microsomes from rats treated with INH and PB also coincided with the $\mathrm{pH}$ optimum for MDA formation by these microsomes (Figures 21 and 24) suggesting that the two processes may be linked. Furthermore, an intact mixed-function oxidase system is required for the formation of 4(R)4-OH-OTA, but not 4(S)-4-OH-OTA or lipid peroxidation. Replacement of NADPH by ascorbate results in the direct chemical reduction of the OTA-Fe3+ complex giving rise to non-enzymatic lipid peroxidation. The ability of ascorbate to stimulate microsomal lipid peroxidation is well known (Ernster and Nordbrand, 1982). In the presence of ascorbate, only the $4(S)$ isomer was formed, suggesting the requirement for an intact mixed function oxidnse system for the formation of the $4(R)$ isomer. The need for cytochrome P-450 in the formation of the $4(R)$ isomer is also substantiated by the observation that no OTA metabolites were detected when liver microsomes from $\mathrm{Co}$-heme pretreated rats were used. A correlation between lipid peroxidation and 4(S)-4-OH-OTA [but not 4(R)-4-OH-OTA] formation is further strengthened by the observation that both processes are remarkably sensitive to inhibition by the iron chelators Desferal and BPS and the antioxidants BHA and DPPD (Table 16). In fact, in the presence of varying concentrations of BHA (Figure 23), both lipid peroxidation and 4(S)-4-OH-OTA formation are inhibited in parallel suggesting that either lipid peroxides are responsible for $4(\mathrm{~S})-4-\mathrm{OH}-\mathrm{OTA}$ formation or that a common species mediates the formation of both.

The inability of microsomes to produce 4(S)-4-OH-OTA in the presence of linoleic acid hydroperoxide (LAHP) suggests that lipid peroxides may not be involved in its formation (Table 18). In contrast, microsomes in the presence of CHP gave rise to substantial amounts of both 4(R)-4-OH-OTA and 4(S)-4-OH-OTA. Cytochrome P-450) is known to function as a peroxidase and the ability of CHP to catalyze the cytochrome P-450-dependent metabolism of xenobiotics has been well documented (Rahimtula and 
O'Brien, 1974; Rahimtula and O'Brien, 1975). A variety of hemoproteins including HRP (Corbett and Corbett, 1987; O'Brien, 1988) and $\mathrm{Hb}$ (Catalano and Ortiz de Montellano, 1987) are also known to oxidize xenobiotics. However, these hemoproteins as well as hematin were unable to catalyze OTA oxidation in the presence of a variety of hydroperoxides suggesting that cytochrome $\mathrm{P}-450$ may be essential (table 18 ).

The lack of substantial inhibition by SOD or catalase suggests that the superoxide anion or $\mathrm{H}_{2} \mathrm{O}_{2}$ are not directly involved in the formation of 4(S)-4OH-OTA or MDA by microsomes from rats treated with INH. Both SOD and catalase increased OTA metabolism by microsomes from rats treated with PCN, possibly by protecting cytochrome P-450 from radical-induced damage. Previously, we have shown (Hasinoff et al., 1990) that the presence of OTA stimulated superoxide and hydroxyl madical formation by liver microsomes. Mannitol, a known scavenger of hydroxyl radicals, did inhibit 4(S)-4-OH-OTA by about $45 \%$ with microsomes from rats treated with INH indicating that hydroxyl radicals may play a role. However, mannitol did not inhibit either the NADPH-dependent (by microsomes from PCN treated rats) or the ascorbate-dependent (by microsomes from INH and PCN treated rats) metabolism of OTA, or lipid peroxidation, indicating that hydroxyl radicals are probably not involved in either of these processes. Gutteridge (1982) showed that hydroxyl radicals do not play a significant role in NADPH-dependent microsomal lipid peroxidation.

The induction of lipid peroxidation (Omar et al., 1990) and active oxygen species (Hasinoff et al., 1990) by OTA can lead to the destruction of cylochrome P-450 which is responsible for 4(R)-4-OH-OTA formation. Desferal, by chelating iron, would prevent the formation of the active oxygen species responsible for lipid peroxidation and thus protect the cytochrome P-450 from destruction. This was indeed observed (Table 16). The fact that Desferal inhibited the formation of 4(S)-4-OH-OTA [but not that of 4(R)-4-OH-OTA] and MDA suggests that free iron is essential in their formation. This, logether with 
observations that cytochrome P-450 is essential and that free (unbound) reactive oxygen species are not involved, suggests that an active $\mathrm{Fe}^{2+-o x y g e n ~ c o m p l e x ~ m a y ~ b e ~ r e s p o n s i b l e ~}$ for both 4(S)-4-OH-OTA formation and lipid peroxidation (see Figure 35 at the end of this chapter). The precise mechanism by which such a hydroxylation occurs is presently not known. We have already demonstrated (Omar et al., 1990) that OTA induces lipid peroxidation by chelating $\mathrm{Fe}^{3+}$ and that the resulting $\mathrm{OTA}-\mathrm{Fe}^{3+}$ chelate is more readily reducible by the flavoprotein NADPH-cytochrome P-450 reductase to the OTA-Fe'2+ complex which, in the presence of oxygen, provides the active species that initiates lipid peroxidation. Cytochrome $\mathrm{P}-450$ is required presumably to facilitate the reduction of the $\mathrm{Fe}^{3+}$ complex to the $\mathrm{Fe}^{2+}$ complex. The exact nature of the initiating species is presently not known and different investigators have implicated the perferryl ion (Tien et al., 1981; Ursini et al., 1989), the ferryl ion (Koppenol, 1985) and the $\mathrm{Fe}^{2+-} \mathrm{O}_{2}-\mathrm{Fe}^{3+}$ complex (Bucher et al., 1983).

Formation of the $4(R)$ and $4(S)$ isomers showed clear difference with respect to $\mathrm{pH}$ optima. The pH optimum for the formation of the $4(\mathrm{R})$ isomer was 6.0 by microsomes from rats treated with $3 \mathrm{MC}$ (Figure 25) and ISF (data not shown), and 6.5 by microsomes from rats treated with $\mathrm{PB}$ (Figure 24), whereas that for the formation of the $4(\mathrm{~S})$ isomer was $7.0-7.5$; the latter coincided with the $\mathrm{pH}$ optimum for lipid peroxidation (Figures $21 \mathrm{~b}$ and $24 \mathrm{~b}$ ). Because of this marked difference in the $\mathrm{pH}$ optima for the formation of the $4(\mathrm{R})$ and $4(\mathrm{~S})$ isomers, OTA metabolism was measured th the two different plis (pH 6.0), optimum for the formation of the $4(R)$ isomer by microsomes from rats treated with 3MC and ISF, and $\mathrm{pH} 7.5$, optimum for the formation of the $4(\mathrm{~S})$ isomer and lipid peroxidation, were chosen). At pH 6.0 (compared to $\mathrm{pH} 7.5$ ), the formation of the $4(\mathrm{R})$ isomer increased by 7.7 and 5.0 fold using microsomes from rats treated with $3 \mathrm{MC}$ and ISF respectively. On the other hand, at $\mathrm{pH} 7.5$, the formation of the $4(\mathrm{~S})$ isomer increased by 
3.4 and 2.7 fold using microsomes from rats treated with ISF and PB respectively as compared to $\mathrm{pH} 6.0$ (Table 19). This increase in 4(R)-4-OH-OTA formation at $\mathrm{pH} 6.0$ (versus pH 7.5) could be due to less ionization of OTA at $\mathrm{pH} 6.0$ which would make it more lipophilic and possibly allow it to access cytochrome P-450 more readily, and/or it could be that less lipid peroxidation is occurring at $\mathrm{pH} 6.0$ and thus cytochrome $\mathrm{P}-450$ is more protected allowing for increased metabolism. Increased lipid peroxidation at $\mathrm{pH} 7.5$ (versus $\mathrm{pH} 6.0$ ) could also account for increased $4(\mathrm{~S})$-4-OH-OTA formation especially if the two processes are linked. Measurement of cytochrome $\mathrm{P}-450$ at the end of an incubation at the two different $\mathrm{pH}$ revealed that at $\mathrm{pH} 6.0$, the percentages of cytochromes P-450 lost were 32,15 and $51 \%$ using microsomes from rats treated with $3 \mathrm{MC}$, ISF and PB, respectively (Table 20). The corresponding MDA amounts formed by microsomes from the same sources were $18.9,8.0$ and $11.8 \mathrm{nmol} / \mathrm{mg}$ protein. The percentages of cytochromes $\mathrm{P}-450$ lost at $\mathrm{pH} 7.4$ were 57,43 and $59 \%$ using microsomes from rats treated with 3MC, ISF and PB, respectively (Table 20). The corresponding MDA amounts formed by microsomes from the same sources were $26.8,17.1$ and $20.2 \mathrm{nmol} / \mathrm{mg}$ protein. The greatest loss of cytochrome P-450 correlated with the highest MDA formed (Table 20). Thus, it seems that lipid peroxidation is an important factor that determines the percentage loss of cytochrome P-450 that in turn affects the metabolism of OTA.

The large increase in the rate of OTA hydroxylation at $\mathrm{pH} 6.0$ as compared to $\mathrm{pH}$ 7.4 using microsomes from rats treated with $3 \mathrm{MC}$ or ISF can be only partially due to the protective effect on cytochrome $\mathrm{P}-450$ at the lower $\mathrm{pH}$. Similarly, an alteration in the $\mathrm{Km}$ of the substrate also appears not to be the critically important factor because the Km of OTA hydroxylation decreased at $\mathrm{pH} 6.0$ using microsomes from rats treated with $3 \mathrm{MC}$, but it increased using microsomes from rats treated with ISF. The most likely reason for the increase in OTA at pH 6.0 using microsomes from $3 \mathrm{MC}$ or ISF treated rats could be that at this $\mathrm{pH}$ the particular isozymes metabolizing OTA (cylochrome P-450 IA1/LA2) undergo a 
conformational change in a manner that enhances OTA hydroxylation. The hydroxylating $\mathrm{Fe}^{3+-O x y g e n}$ complex is probably brought into close proximity of the substrate at the site where hydroxylation occurs. Such a conformational change due to lowering of the $\mathrm{pH}$ may not favor the hydroxylation of other substrates which bind to the enzyme differentially. Also, other isozymes (for example, IIB1) having a different apoenzyme structure would undergo conformational changes that may not be conducive to OTA hydroxylation. 


\subsection{Role of cytochrome P-450 in OTA-induced toxicity.}

The hepatic microsomal cylochrome P-450 system (mainly cytochrome P-450

families I, II, III and IV) metabolizes a large number of xenobiotics (chemicals, drugs, and environmental contaminants). Most metabolism leads to detoxification of the xenobiotics and their rapid excretion, but some compounds are known to be activated to intermediates that are toxic and/or carcinogenic. For example, aflatoxin $\mathbf{B}_{1}$ is activated by $3 \mathrm{MC}$ inducible cytochrome P-450-AFB (Fukuhara et al., 1989 and 1990). Activation of dimethylnitrosamine and tobacco-smoke-related nitrosamines to more genotoxic and carcinogenic products by different cytochromes P-450 (particularly IIE1 and IIA6) has also been shown (Yamazaki et al., 1992). Enalapril maleate, a drug used to treat hypertension, exerts greater hepatotoxicity upon induction of cytochrome P-450 IIIA by PCN (JurimaRomet and Huang, 1992). Therefore, the cytochrome P-450 system is important in drug detoxification as well as in drug activation to mutagens, carcinogens and toxins, and genetically determined differences in the activity of these enzymes can influence individual susceptibility to adverse drug reactions, drug induced diseases and certain types of chemically induced cancers. Debrisoquine is used as a test drug to classify individuals into phenotypes based on their metabolic handling of it. There are two main phenotypes: the rapid acetylator/extensive metabolizer and slow acetylator/poor metabolizer (Eichelbaum et al., 1992).

Other important factors that alter the cytochrome P-450 isozyme profile include alcohol consumption, smoking and a variety of drugs and xenobiotics. Altering the cytochrome P-450 profile in the liver may result in serious consequences. Ethanol is known to induce cytochrome P-450 IIE1 (Ekstrom and Ingelman-S undberg, 1989), therefore the fate of a certain drugixenobiotic would differ in a person who consumes alcohol from that in a person who does not consume alcohol. One might be more prone to drug toxicity than the other. It was suggested that the bioactivar? of of $\mathrm{N}$-nitroso- 
dimethylamine (NDMA) by cytochrome P-450 IIE1 induced by chronic alcohol consumption plays a critical role in the hepatotoxicity of NDMA (Ma et al., 1991). Also, serious hepatotoxicity may develop in chronic alcoholics while they are taking therapeutic doses of acetaminophen. The mechanism of increased susceptibility involves induction of cytochrome P-450 by alcohol, and the depletion of hepatic glutathione reserves, both of which can result from chronic alcohol ingestion and both of which affect acetaminophen metabolism (Rex and Kumar, 1992). Antrila et al. (1991) suggested that smoking and peripheral type of eancer are related to high levels of pulmonary cytochrome P-450 IA I/2 (which is involved in metabolic activation of polycyclic aromatic hydrocarbons and aromatic amines) in lung cancer patients.

Ochratoxin A is primarily a nephrotoxin. We therefore reasoned that if induction of hepatic cytochrome P-450 leads to more rapid detoxification of OTA and its subsequent clearance, then the kidneys would be exposed to less toxin and for a shorter time. This should result in less damage to the kidneys as compared to the non-induced group. On the other $\mathrm{b}, \mathrm{nd}$, if hepatic cytochrome P-450 induction results in the activation of OTA to a more toxic metabolite then the kidneys would be exposed to a more toxic form of the toxin and would show more damage compared to the kidneys from a non-induced group. We therefore compared the effect of OTA administration to PB-treated rats (liver cytochrome P450 induced) and to control rats. Excretion of renal enzymes in the urine is considered to be a sensitive, non-invasive indicator of renal toxicity and damage (Price, 1982; Ngaha and Plummer, 1977). Figures 28 and 29 show urine alkaline phosphatase and8-glutamyl transferase levels both in PB treated and control rats on day 0 (before OTA treatment) and days 1 - 5 after OTA treatment. Levels of both enzymes were elevated on the fourth day after OTA treatment in control rats, but not in PB treated rats indicating that pretreatment with PB protected the kidneys. In the case of $\gamma$-glutamyl transferase, enzyme levels were also elevated on days 1,2 and 3 for control group and on days 2 and 3 for PB group. 
OTA being an anion is known to be transported into the kidneys through the anion transport channel (Sokol et al., 1988). It is also known that OTA inhibits the transport of the anion PAH in kidney cortex slices (Sokol et al., 1988). From our results, the anion transport system was not affected by the PB treatment (Figure 30) since PAH transport was inhibited by $55-60 \%$ in OTA treated rats compared to control rats, and there was no significant difference in the inhibition of PAH transpsort in OTA treated rats and in PB pretreated rats treated with OTA (Figure 30). Thus, the effect exerted by PB on OTA is more likely to be enhanced metabolism and faster elimination through induction of hepatic cytochrome $\mathrm{P}-450$. This is more evident from the results obtained from the in vivo experiment using [ ${ }^{3 H}$ ]OTA. Quantitation of OTA in different tissues and body fluids is helpful because it can provide useful information about the amount absorbed, bound to serum albumin and excreted in the urine. Figure 31 shows OTA levels in serum and urine of $\mathrm{PB}$ treated rats and control rats $6 \mathrm{hr}$ after OTA administration. Even though it was not significantly different, there was a tendency of increased OTA concentration in the serum of control rats compared to PB treated rats (OTA is known to bind tightly to senum albumin; Chu, 1971). Moreover, there was an increase in OTA concentration in the urine of the PB treated rats compared to control rats indicating that OTA was absorbed, metabolized and excreted faster in PB rats as compared to control rats. OTA levels in the tissues (liver, kidney, stomach, the four portions of small intestine and cecum), and in the gut contents are shown in Figures 32, 33 and 34. Higher OTA levels were found in the gut contents (contents of stomach, small intestine and cecum) of control rats compared to PB treated rats again indicating that OTA was absorbed, metabolized and excreted faster in the PB rats. More OTA was found in the four portions of the small intestinal tissues in the control rats; this may have been because there was more unabsorbed OTA in the intestinal contents of the control rats. The high OTA levels in cecum contents might represent, in part, excretion of OTA and metabolites ir bile since OTA is known to undergo enterohepatic circulation 
(Fuchs et al., 1988).

The above results suggest that the induction of cytochrome P-450 of fers protection against OTA toxicity. In support of this view. Hutchison et al. (1971) showed that 4-OHOTA was non-toxic to rats at doses up to $40 \mathrm{mg} / \mathrm{kg}$. Further support came from Met ui ct al. (1985) who showed that the acute toxicity of OTA was reduced in mice by pretreatment with PB for 1 week, and the $\mathrm{LD}_{50}$ increased to 1.5-2.0 times control. They also observed increased OTA concentration (2-fold) in the bile extract of PB-pretreated mice (in which also they observed un known metabolite) compared to control. In contrast, OTA concentration in the urine extract was decreased to one-third of control. Morol et al. (1985) suggested that a change in the metabolism of OTA could cause the decrease in the toxicity of OTA in PB-pretreated mice. In other words, OTA hydroxylation is more likely to be a detoxification pathway. Thus, it appears that OTA itself is toxic rather than a hydroxylated metabolite. The kidney compared with the liver contains much lower activities of cytochromes P-450 enzyme system, thus preventing it from effectively detoxifying OTA. In the absence of rapid OTA metabolism in the liver, the kidney would be exposed to higher levels of OTA and thus suffer greater toxicity. In addition, OTA is transported into the kidney via the anion transport channel (Sokol et al., 1988) thus incrensing its concentration in that organ. These reasons may account for OTA being more nephrotoxic than hepatotoxic.

\section{Relevance to human toxicity.}

OTA has been shown to be nephrotoxic and hepatotoxic to a varicty of animals and is strongly suspected of being the main etiologic agent responsible for Balkan endemic nephropathy and associaled urinary tract tumors in humans. The different cytochrome $\mathrm{P}$ 450 isozymes are mainly responsible for oxidizing the various structurally diverse chemicals to which an organism is exposed to readily excretable hydrophillic metabolites. 
Many of these isozymes are highly inducible by a variety of xenobiotics and their induction leads to enhanced metabolism of their substrates. Toxicity to a particular tissue/organ is most often the end result of that tissue/organ being exposed for an appropriate length of time to a sufficient concentration of a toxic chemical or its reactive metabolite.

My results suggest that OTA is itself toxic since its enhanced metabolism in PB treated (cytochrome P-450 induced) rats protected against nephrotoxicity. If increased cytochrome P-450 levels protect against OTA nephrotoxicity in bumans, then diets rich in cruciferous vegetables (cabbage, brussels, sprouts), broiled foods or w-3 fatty acids which induce cytochrome P-450 should protect against OTA nephrotoxicity. Discounting their side effects, cigarette smoke and a variety of cytochrome P-450 inducing drugs should also exert a protective effect. It is clear from my studies that several cytochrome P-450 isozymes are able to metabolize OTA.

High dietary fat is known to reduce absorption of chemicals and other nutrients from the gut. Studies carried out in our laboratory with OTA dissolved in corn oil versus bicarbonate have confirmed this. A high fat diet would thus be expected to increase the half-life of OTA in the body thus increasing the risk of tissue damage. In humans, therefore, a low fat diet might protect against OTA nephrotoxicity (reduce OTA toxicity, but not fully protect against it) by enhancing its absorption through the gut and subsequent elimination.

In conclusion, avoiding or at least minimizing exposure to OTA would offer the bcst protection against nephrotoxicity. However, a diet low in fat and rich in cytochrome P-450 inducers should protect against OTA nephrotoxicity.

This study should be important to humans and of relevance because humans have similarities in a number of their cytochromes P-450 to those of rats. Mostly, they have more than $70 \%$ homology between them on the level of amino acid sequence and DNA (see Table 21 at the end of this chapter). 


\section{CONCLUSIONS}

My data provide evidence for the stimulatory effect of cytochrome P-450 on OTAinduced lipid peroxidation in a reconstituted system, and strongly implicate cytochrome P. 450 role in OTA-induced lipid peroxidation in microsomes.

The results also show that OTA is metabolized by the main cytochrome P-450 isoforms IA1/LA2, IIB1 and IIIA 1/IIIA2. This conclusion is based on studies using 1) specific cytochrome P-450 inducers, 2) specific substrates, 3) specific inhibilors and 4) monoclonal antibodies against specific cytochrome P-450 isoforms.

The results from in vivo experiments suggest a protective effect of PB against OTA toxicity, probably through enhanced metabolism and detoxification due to cytochrome P. 450 induction. 
Key words for indexing:

Ochratoxin, lipid peroxidation, cytochrome P-450, inducers, inhibitors, monoclonal antibodies, rat, platelets, aggregation, secretion. 
Figure 35 Scheme representing the possible mechanism of $4(R)$ - and 4(S)-4-OH-OTA formation and of OTA-stimulated lipid peroxidation. 

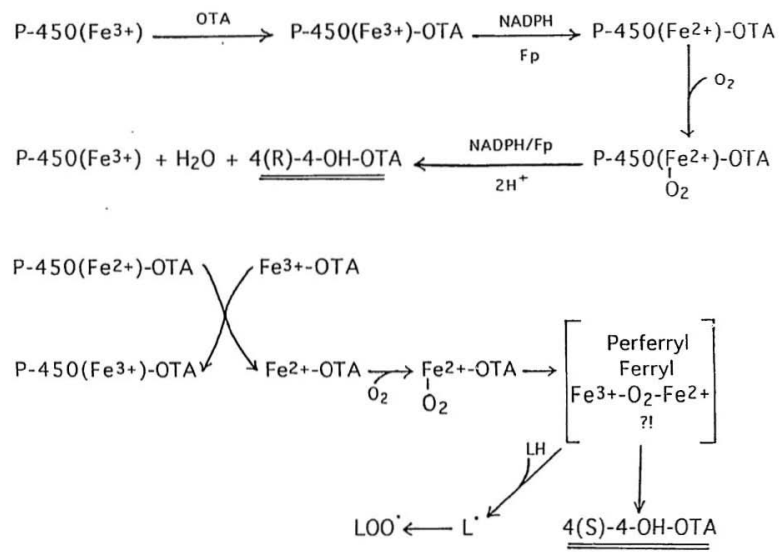
Table 21. Sequential homology between rat and human P-450 forms (Adapted from Soucek and Gut., 1992).

\begin{tabular}{|c|c|c|c|}
\hline \multicolumn{2}{|c|}{ Cyt. P-450 form } & \multicolumn{2}{|c|}{ Sequential homology (\%) } \\
\hline Rat & Human orthologue & DNA & amino acid \\
\hline & & & \\
\hline CYPLAI & CYPIA1 & 80 & 78 \\
\hline CYPLA2 & CYPIA2 & 75 & 70 \\
\hline CYPIB1 & CYРПВ6 & 78 & 74 \\
\hline CYPIIE1 & CYPШE1 & 75 & 78 \\
\hline CYPIIAI & CYPIIIA3 & $-a$ & 78 \\
\hline CYPIIIA2 & CYPШAS & -3 & 71 \\
\hline CYPIVAI & CYPIVA9 & $-\mathbf{a}$ & $-a$ \\
\hline
\end{tabular}

a Data not available 


\section{REFERENCES}

Abrahamson D, Mills JT, and Boycott BR. Mycotoxins and mycoflora in animal fcedstuffs in Western Canada. Can .J. Comp. Med. 47: 23-26, 1983.

Albano E, Poli G, Tomasi A, Bini A, Vannini V and Dianzani MU. Toxicity of 1,2dibromomethane in isolated hepatocytes: Role of lipid peroxidation. Chem. Biol. Interact. 50: 255-265, 1984.

Aleo MD, Wyatt RD and Schnellmann RG. Mitochondrial dysfunction is an early event in ochratoxin A but not oosporein toxicity to rat renal proximal tubules. Toxicol. Appl.

Pharmacol. 107: 73, 1991.

Anttila S, Hietanen E, Vainio H, Camus A-M, Gelboin HV, Park SS, Heikkila L, Karjalainen $\mathrm{A}$ and Bartsch $\mathrm{H}$. Smoking and peripheral type of cancer are related to high levels of pulmonary cytochrome P-450 IA in lung cancer patients. Int. J. Cancer 47: 681$685,1991$.

Atkinson RM, Currie JP, Davis B, Pratt DAH; Sharpe HM and Tomich EG. Acute toxicity of cephaloridine, an antibiotic derived from cephalosporin C. Toxicol. Appl. Pharmacol. 8: $398-406,1966$.

Aust SD. Sources of iron for lipid peroxidation in biological systems. In: Oxygen Radical and Tissue Injury; Proceedings of an Upjohn Symposium (Halliwell B, Ed.), pp. 27-33, Allen Press, Kansas, 1988.

Aust SD and Svingen BA. The role of iron in enzymatic lipid peroxidation. In: Free Radicals in Biology (Pryor W A, Ed.), Vol. 5, pp. 1-28, Academic Press, New York, 1982.

Balzer I, Bogdanic C and Muzic S. Natural contamination of corn (Zea mays) with mycotoxins in Yugoslavia. Ann. Nutr. Aliment. 31: 425-430, 1977.

Barber DJW and Thomas JK. Reactions of radicals with lecithin bilayers. Radiation Res. 74: $51-65,1978$. 
Bartlett GR. Phosphorous assay in column chromatography, J. Biol. Chem. 234:466468, 1959.

Bartsch H, Malaveille C, Camus AM, Mrtel-Planche G, Brun G, Hautelfeville A, Sabadie N, Barbin A, Kuoki T, Drevon C, Piccoli A and Montesano R. Validation and comparative studies on 180 chemicals with $S$. typhimurium strains and V79 Chinese hamster cells in the presence of various metabolizing systems. Mutation Res. 76: 1-50, 1980.

Bauer $\mathbf{J}$ and Gareis M. Ochratoxin A in der Nahrungsmittelkette. Z. Veterinarmed. B. 34: 613-627, 1987.

Belogui $O$ and Cederbaum AI. Prevention of microsomal products of hydroxyl radicals, but not lipid peroxidation, by the glutathione-glutathione peroxidase system. Biochem. Pharmacol. 35: 2663-2669, 1986.

Bendele AM, Carlton WW, Krogh P and Lillehoj EB, Ochrutoxin A carcinogenesis in the (C57BL/6JXC3H)F1 mouse. J. Natl. Cancer Inst. 75: 733-739, 1985.

Benedetti $A$, Comporti $M$ and Esterbauer $H$. Identification of 4-hydroxynonenal as a cytotoxic product originating from the peroxidation of liver microsomal lipids. Biochim. Biophys. Acta 620: 281-296, 1980.

Berndt WO and Hayes AW. In vivo and in vitro changes in renal function caused by ochratoxin A in the rat. Toxicol. 12: 5-7, 1979.

Berndt WO, Hayes AW and Phillips RD. Effects of mycotoxins on renal function: Mycotoxic nephropathy. Kidney Intl. 18: 656-664, 1980.

Bond EJ and De Matteis FA. Biochemical changes in rat liver after administration of carbon disulphide, with particular reference to microsomal changes. Biochem. Pharmacol. 18: 2531-2549, 1969.

Boorman G, NTP Technical Report on the Toxicology and Curcinogenesis Studies of 
Ochratoxin A. NTP TR 358, NIH Publication No. 88-2813, Research Triangle Park, North Carolina, 1988.

Braughler JM, Duncan LA and Chase RL. The involvement of iron in lipid peroxidation. Importance of ferric to ferrous ratios in initiation. J. Biol. Chem. 261: 10282-10289, 1986.

Brown MH, Szczech GM and Purmalis BP. Teratogenic and toxic effects of ochratoxin A in rats. Toxicol. Appl. Pharmacol. 37: 331-338, 1976.

Brownlee NR, Hunter JJ, Panganamala RV and Comwell DC. Role of Vitamin E in glutathione induced oxidatative stress: Methemoglobin, lipid peroxidation, and hemolysis. J. Lipid Res. 18: 635-644, 1977.

Bruch RC and Thayer WS. Differential effect of lipid peroxidation on membrane fluidity as determined by electron spin resonance probes. Biochim. Biophys. Acta 733: 216-222, 1983.

Bucher JR, Tien $M$ and Aust SD. The requirement for ferric in the initiation of lipid peroxidation by chelated ferrous iron. Biochem. Biophys. Res. Commun. 111: 777-784, 1983.

Burke MD and Mayer RT. Differential effects of phenobarbitone and 3-methylcholanthrene induction on the hepatic microsomal metabolism and cytochrome P-450-binding of phenoxazone and a homologous series of its $\mathrm{n}$-alkyl ethers (alkoxyresorufins). Chem. Biol. Interact. 45: 243-258, 1983.

Burke MD, Thompson S, Elcombe CR, Halpert J, Haaparanta T and Mayer RT. Ethoxy-, pentoxy- and benzyloxyphenoxazones and homologues: A series of substrates to distinguish between different induced cytochrome P-450. Biochem. Pharmacol. 34(18): 3337-3345, 1985.

Casini AF, Pompella A and Comporti M. Liver glutathione depletion induced by bromobenzene, iodobenzene and diethyl maleate poisoning and its relation to lipid 
peroxidation and necrosis. Am. J. Pathol. 118: 225-237, 1985.

Catalano CE and Ortiz de Montellano PR. Oxene transfer, electron abstraction and cooxidation in the epoxidation of stilbene and 7,8 dihydroxy-7,8-dihydrobenzolalpyrene by hemoglobin. Biochemistry 26: 8373-8380, 1987.

Catheart R, Schwiers E and Ames BN. Detection of picomole levels of hydroperoxides using a fluorescent dichlorofluoresccin assay. Anal. Biochem. 134: 111-116, 1983.

Chance $\mathrm{B}$, Sies $\mathrm{H}$ and Boveris $\mathrm{A}$. Hydroperoxide metabolism in mammalian organs. Physiol. Rev. 59: pp. 527, 1979.

Chelkowski J, Golinski P and Szebiotko K. Mycotoxins in cereal grain. Il. The fate of ochratoxin A after processing of wheat and barley grain. Nahrung 25: 423-426, 1981.

Chu FS. Interaction of ochratoxin A with bovine serum albumin. Arch. Biochim. Biophys. 147: 359-366, 1971.

Cojocel C, Hannemann J and Baumann K. Cephaioridine-induced lipid peroxidation initiated by reactive oxygen species as a possible mechanism of cephaloridine nephrotoxicity. Biochim. Biophys. Acta 834: 402-410, 1985a.

Cojocel C, Lacschke KH, Inselmann $\mathrm{G}$ and Baumann K. Inhibition of cephaloridineinduced lipid peroxidation. Toxicology 35: 295-305, $1985 \mathrm{~b}$.

Comporti M. Biology of disease, lipid peroxidation and cellular danage in toxic liver injury. Lab. Invest. 53: 599-623, 1985.

Comporti $\mathrm{M}$, Saccocci $\mathrm{C}$ and Dianzani MU. Effect of $\mathrm{CCl}_{4}$ in vitro and in vivo on lipid peroxidation of rat liver homogenates and subcellular fractions. Enzymologia 2\%: 185 204, 1965.

Connole MD, Blaney BJ and Mcewan T. Mycotoxins in animal feeds and toxic fungi in Queensland 1971-1980. Aust. Vet.J. 57: 314-318, 1981. 
Corbet MD and Corbett BR. HRP-catalyzed bioactivation of carcinogenic hydroxamic acids. The greater reactivity of glycolyl- versus acetyl-derived hydroxamic acids. Chem. Biol. Interact. 63: 249-264, 1987.

Corongiu FP and Milia A. An improved and simple method for determining diene conjugation in autoxidized polyunsaturated fatty acids. Chem. Biol. Interact. 44: 289-297, 1983.

Creppy EE, Chakor K, Fisher MJ, and Dirheimer G. The mycotoxin ochratoxin A is a substrate for phenylalanine hydroxylase in isolated rat hepatocytes and in vivo. Arch. Toxicol. 64: 279-284, 1990.

Creppy EE, Kane A, Dirheimer G, Lafarge-Frayssinet C, Mousset S and Frayssinet C. Genotoxicity of ochratoxin A in mice: DNA single-strand break evaluation in spleen, liver and kidncy. Toxicol. Lett. 28: 29-35, 1985.

Creppy EE, Kane A, Giessen-Crouse E, Roth A, Roschenthaler R and Dirheimer G. Effect of ochratoxin A on enzyme activities and macromolecules synthesis in MDCK cells. Arch. Toxicol. Suppl. 9: 310-314, 1986.

Creppy EE, Kern D, Steyn PS, Vleggaar R, Roschenthaler R and Dirheimer G. Comparative study of the effect of ochratoxin $A$ analogues on yeast aminoacyl-t-RNA synthetases and on the growth and protein synthesis of hepatoma cells. Toxicol. Lett. 19: 217-224, 1983a.

Creppy EE. Lorkowski G, Roschenthaler R and Dirheimer G. Kinetics of the immunosuppressive action of ochratoxin A on mice. In Proceedings, $V$ International IUPAC Symposiun Mycotoxins and Phycotoxins, Sept. 1-3, 1982, Vienna, pp. 289-292. Austrian Chem Soc, Vienna, 1982.

Creppy EE, Roschenthaler $R$ and Dirheimer $G$. Inhibition of protein synthesis in mice by ochratoxin A and its prevension by phenylalanine. Food Chem. Toxicol. 22: 883-886, 1984. 
Creppy EE, Stormer FC, Kern D, Roschenthaler R and Dirheimer G. Effects of ochratoxin A metabolites on yeast phenylalanyl-tRNA synthetase and on the growth and in vivo protein synthesis of hepatome cells. Chem. Biol. Interact. 47: 239-247, 1983b

Csallany AS, Dergvan M, Manwaring JD and Addis PB. Free malondialdehyde determination in tissues by high performance liquid chromatography. Anal. Biochem. 142: $277-283,1984$.

Curtis MT, Gilfor D and Farber J. Lipid peroxidation increnses the molecultr order microsomal membranes. Arch. Biochem. Biophys. 235: 644-649, 1984.

Dahle LK, Hill EG and Holman RT. The thiobarbituric acid reaction and the autoxidation of polyunsaturated fatty acid methyl esters. Arch. Biochem. Biophys. 98: 253-261, 1962.

de Groot $\mathrm{H}$, Noll $\mathrm{T}$ and Tolle $\mathrm{T}$. Loss of latent activity of liver microsomal membrane enzymes evoked by lipid peroxidation. Studies of nucleoside diphosphatase, glucose-6phosphatase, and UDP glucuronyltransferase. Biochim. Biophys. Acta 815: 91-96, 1985.

De Scott B. Toxigenic fungi isolated from cereal and legume products. Mycopath. Mycol. Appl. 25: 213-222, 1965.

Dianzani MU and Torrielli MV. Lipid peroxidation in ethanol-induced liver damage. The problem of its possible relevance in both acute and chronic alcoholism. Med. Biol. Environ. 9: 177-190, 1981.

Dianzani MU and Ugazio G. Lipid peroxidation. In: Biochemical Mechanism.s of Liver Injury (Slater T F, Ed.), pp. 669-707, Academic Press, London, 1978.

Dianzani MU. Lipid peroxidation in ethanol poisoning: A critical reconsideration. Alcohol and alcoholism 20: 161-173, 1985.

Dietrich DR and Swenberg JA. Renal carcinogenesis. In: Toxicology of the Kidney (Hlook JB and Goldstein RS, Eds.) pp. 495-537, Raven Press, New York, 1993. 
Dillard CJ, Dumelin EE and Tappel AL. Effect of dietary vitamin E on expiration of pentane and ethane by the rat. Lipids 12: 109-114, 1977.

Dominguez OV and Samuels LT. Mechanism of inhibition of adrenal steroid 11-betahydroxylase by methopyrapone (metopirone). Endocrinology 73:304-309, 1963.

Drummond GS and Kappas A. The cytochrome P-450- depleated animal: An experimental model for in vivo studies in chemical biology. Proc. Natl. Acad. Sci., USA 79: 2384-2388, 1982.

Dwivedi P and Burns RB. Effect of ochratoxin A on immunoglobulins in broiler chicks. Res. Vet. Sci. 36: 117-121, 1984.

Eichelbaum M, Kroemer HK and Mikus G. Genetically determined differences in drug metabolism as a risk factor in drug toxicity. Toxicol. Lett. 64/65: 115-122, 1992.

Eichenberg K, Bohni P, Winterhalter KH, Kawato S and Richter C. Microsomal lipid peroxidation causes an increase in the order of the membrane lipid domain. FEBS Lett. 142: $59-62,1982$.

Ekstrom T and Hogberg J. Chloroform induced glutathione depletion and toxicity in freshly isolated hepatocytes. Biochem. Pharmacol. 29: 3059-3065, 1980.

Ekstrom $\mathrm{G}$ and Ingelman-Sundberg M. Cytochrome P-450 dependent lipid peroxidation in reconstituted membrane vesicles. Biochem. Pharmacol. 33: 2521-2525, 1984.

Ekstrom $\mathrm{G}$ and Ingelman-Sundberg $\mathrm{M}$. Rat liver microsomal NADPH-supported oxidase activity and lipid peroxidation dependent on ethanol-inducible cytochrome P-450 (P-450 IIE1), Biochem.Pharmacol. 38: 1313-1319, 1989.

El-Banna AA and Scott PM. Fate of mycotoxins during processing of foodstuffs. III. Ochratoxin A during cooking of faba beans (Vicia faba) and polished wheat. J. Food. Prot. 47: $189-192,1984$. 
Ernster L and Nordenbrand K. Microsomal lipid peroxidation. Mechanisms and some biological implications. In: Lipid peroxides in biology and medicine (Yagi $\mathrm{K}, \mathrm{Ed}$. ), pp. 55-79, Academic Press, New York, 1982.

Fee JA. Is superoxide toxic and are superoxide dismutases essential for aerobic life. In: Oxygen and Oxy-Radicals in Chemistry and Biology (Rodgers M A J and Powers E L, Eds.), 205-221, Academic Press, New York, 1981.

Ferrali M, Fulceri R, Benedetti A and Comporti M. Effects of carbonyl compounds (4hydroxyalkenals) originating from the peroxidation of liver microsomal lipids on microsomal enzyme system of the liver. Res. Commun. Chem. Pathol. Pharmacol. 30: pp. 99, 1980.

Fisher GJ, Fukushima and Gaylor JL. Isolation, purification and properties of a unique form of cytochrome P-450 in microsomes of isosafrole-treated rats. J. Biol. Chem. 256: 4388-4394, 1981.

Folch I, Lees $\mathrm{M}$ and Stanley GM. A simple method for isolation and purification of total lipids from animal tissues. J. Biol. Chem. 226: 497-509. 1956.

Fong K-L, McCay PB, Poyer IL, Keele BB and Misra H. Evidence that peroxidation of lysosomal membranes is initiated by hydroxyl radicals produced during flavin enzyme activity. J. Biol. Chem. 248: 7792-7797, 1973.

Fournel S, Magdalou J, Pinon P and Siest G. Differential induction of drug-metabolizing enzymes after treatment with hypolipidaemic agents. Xenobiotica 17: 445-457, 1987.

Frankel EN and Neff WE. Formation of malonaldehyde from lipid oxidation products. Biochim. Biophys. Acta. 754: 264-270, 1983.

Franklin MR. Inhibition of mixed-function oxidations by substrates forming reduced cytochrome P-450 metabolic-intermediate complexes. Pharmacol. and Ther. 2: 227-245, 1977. 
Fridovich 1. Superoxide dismutase. In: Molecular Mechanisms of Oxygen Activation (Hayaishi O, Ed.), pp. 453, Academic Press, New York, 1974.

Fuchs R, Radic B, Peraica M, Hult K and Plestina R. Enterohepatic circulation of ochratoxin A in rats. Periodicum Biologorum 90: 39-42, 1988.

Fukuhara M, Mizokami K, Sakaguchi M, Nimura Y, Kato K, Inouye S and Takanaka A. Aflatoxin B 1-specific cytochrome P-450 isozyme (P-450-AFB) inducible by 3methylcholanthrene in golden hamsters. Biochem. Pharmacol. 39:463-469, 1990.

Fukuhara M, Nohmi T, Mizokami K, Sunouchi M, Ishidate M, Jr. and Takanaka A. Characterization of three forms of cytochrome P-450 inducible by 3-methylcholanthrene in golden hamster livers with special reference to aflatoxin B1 activation. J. Biochem. 106:253-258, 1989.

Galtier P. Devenir de l'ochratoxine A dans l'organisme animal. I. Transport sanguin de la toxine chez le rat. Ann. Rech. vet. 5: 311-318, 1974.

Galtier $\mathrm{P}$ and Alvinerie M. In vitro transformation of ochratoxin A by animal microbial floras. Ann. Rech. vet. 7: 91-98, 1976.

Galtier P, Charpenteau J-L, Alvinerie M and Labouche C. The phamacokinetic profile of ochratoxin $\mathrm{A}$ in the rat after oral and intravenous administration. Drug Metabolism and Disposition 7(6):429-43`, 1979.

Galtier $P$, Jemmali $M$ and Larrieu $G$. Enquete sur la presence eventuelle d'aflatoxine et d'ochratoxine et dans des mais recolte en France en 1973 et 1974 . Ann. Nutr. Aliment. 31: $381-389,1977 \mathrm{~b}$.

Gayarthri AK and Padmanban G. Biochemical effects of 3,5-diethoxycarbonyl-1,4dihydrocollidine in mouse liver. Biochem. Pharmacol. 23: 2713-2725, 1974.

Gelboin HV, Park SS and Battula N. DNA recombinent and monoclonal antibody directed methods for determining cytochrome P-450 specificity. Biochem. Pharmacol. 37: 98-102, 
1988.

Goldstein RS, Pasino DA, Hewitt WR and Hook JB. Biochemical mechanisms of cephaloridine nephrotoxicity: Time and concentration dependence of peroxidative injury. Toxicol. Appl. Pharmacol. 83: 261-270, 1986.

Golenski P and Grabarkiewicz-Szczesna J. The first Polish of the detection of ochrntoxin A residues in human blood. Rocz. Panstw. Zakl. Hig. (English Abstract) 36: 378-381, 1985.

Gonzalez FJ. Human cytochrome P-450; problems and prospects. TiPS Revicws 13:346$352,1992$.

Graves PE, Kaminsky LS and Halpert J. Evidence for functional and structural multiplicity of pregnenolone-16-carbonitrile-inducible cytochrome P-450 isozymes in rit liver microsomes. Biochem. 26: 3887-3894, 1987.

Guengerich FP. Oxidation of toxic and carcinogenic chemicals by human cylochrome P. 450 enzymes. Chem. Res. Toxicol. 4: 391-407, 1991.

Guengerich FP. Enzymatic oxidation of xenobiotic chemicals. Crit. Rev. Biochem. Mol. Biol. 25: 97-153, 1990.

Guengerich FP, Wang P and Davidson N. Estimation of isozymes of microsomal cytochrome P-450 in rats, rabbits and humans using immunochemical staining coupled with SDS-polyacrylamide gel electrophoresis, Biochem. 21: 1698-1706, 1982a.

Guengerich FP, Dannan GA, Wright ST, Martin MV and Kaminsky LS. Purification and characterization of liver microsomal cytochromes P-450: Electrophr retic, spectral, catalytic and immunochemical properties and inducibility of eight isozymes isolated from rats treated with phenobarbital or B-naphthoflavone. Biochem. 21: 6019-6030, 1982b.

Gutteridge JMC. Lipid peroxidation: Some problems and concepts. In: Oxygen Radical and Tissue Injury; Proceedings of an Upjohn Symposium (Ed. Halliwelll B), pp. 9-19, 
Allen Press, Kansas, 1988.

Gutteridge JMC. The protective action of superoxide dismutase on metal ion catalyzed peroxidation of phospholipids. Biochem. Biophys. Res. Commun. 77: 379-386, 1977.

Gutteridge JMC. The role of superoxide and hydroxyl radicals in phospholipid peroxidation catalyzed by iron salts. FEBS Lett. 150; 454-458, 1982.

Gutteridge JMC. Ferrous-EDTA-stimulated phospholipid peroxidation. A reaction changing from alkoxyl-radical to hydroxyl-radical dependent initiation. Biochem. J. 224: 697-701, 1984.

Guzelian PS and Swisher RW. Degradation of cytochrome P-450 heme by carbon tetrachloride and 2-allyl-2-isopropylacetamide in rat liver in vivo and in vitro: Involvement of non-carbon monoxide-forming mechanisms. Biochem. J. 184: 481-489, 1979.

Hafeman DG and Hoekstra WG. Protection against carbon tetrachloride-induced lipid peroxidation in the rat by dietary vitamin E, Selenium and methionine as measured by ethan evolution. J. Nutr. 107: 656-665, 1977.

Halliwell B. Free radicals, oxygen toxicity and aging. In: Age Pigments (Sohal R S, Ed.), pp. 1-62, Elsevier/North Holland Biomedical Press, Amsterdam, 1981.

Halliwell B and Gutteridge JMC. Oxygen free radical and iron in relation to biology and medicine: Some problems and concepts. Arch. Biochem. Biophys. 246: 501-514, 1986.

Halliwell B and Gutteridge JMC. The importance of free radicals and catalytic metal ions in human disease. Mol. Aspects Med. 8: 89-193, 1985.

Halpert JR, Balfour C, Miller NE and Kaminsky LS. Dichloromethyl compounds as mechanism-based inactivators of rat liver cytochrome P-450 in vitro. Mol. Pharmacol. 30: 19-24, 1986.

Hanson LK, Eaton WA, Sligar SG, Gunsalus IC, Gouterman M and Connell CR. Origin 
of the anomalous soret spectra of carboxycytochrome P-450. J. Am. Chem. Soc. 98: 2672-2674, 1976.

Harwig J, Ochratoxin A and related metabolites. In: Mycotoxins (Purchase IFH, Ed.), pp. 345-368. Elsevier, Amsterdam, 1974.

Hasinoff BB, Rahimtula AD and Omar RF. NADPH- cytochrome P-450 reductase promoted hydroxyl radical production by iron(III)-ochratoxin A complex. Biochim. Biophys. Acta 1036: 78-81, 1990.

Haubeck H-D, Lorkowski G, Kolsch E and Roschenthaler R.Immunosuppression by ochratoxill A and its prevention by phenylalanine. Appl. Environ. Microbiol. 41: 1040)-1042, 1981.

Hayes AW, Hood RD and Lee HL. Teratogenic effects of ochratoxin A in mice. Teratology 9(1): 93-97, 1974.

Hennig A, Fink-Gremmels $\mathbf{J}$ and Leistner L. Mutagenicity and effects of ochratoxin A on the frequency of sister chromatid exchange after metabolic activation. In: Mycotoxins, endemic nephropathy and urinary tract tumors (Castegnaro M, Plestina R, Dirheimer G, Chemozemsky IN and Bartsch H, Eds.), pp. 255-260, Lyon, Intemational Agency for Research on Cancer, IARC, 1991.

Hietanen E, Malaveille C, Camus A-M, Bereziat J-C, Brun G, Castegnaro M, Michelon J, Idle JR and Bartsch $\mathrm{H}$. Interstrain comparison of hepatic and renal microsomal carcinogen metabolism and liver $\mathrm{S} 9$ mediated mutagenicity in DA and Lewis rats phenotyped as poor and extensive metabolizers of debrisoquine. Drug Metabolism Disposition 14: 118-126, 1986.

Hictanen E, Bartsch H, Bereziat J-C, Castegnaro $\mathrm{M}$ and Michelon J. Characterization of the cytochrome P-450 isozyme that metabolizes ochratoxin A, using metabolic inducers, inhibitors and antibodies. In: Mycotoxins, endemic nephropathy and urinary tract tumors (Castegnaro M, Plestina R, Dirheimer G, Chernozemsky IN and Bartsch H, Eds.), pp. 297-304, Lyon, International Agency for Research on Cancer, IARC, 1991. 
Hochstein P, Nordenbrand $K$ and Emster L. Evidence for the involvement of iron in the ADP-activated peroxidation of lipid in microsomes and membranes. Bischem. Bioplys. Res. Commun. 14: 223-238, 1964.

Hochstein P and Ernster L. ADP-activated lipid peroxidation coupled to the TPNII oxidase system of microsomes. Biochem. Biophys. Res. Commun. 12: 388-394, 1963.

Hogberg J, Bergstrand A and Jakobasson SV. Lipid peroxidation of rat liver microsomes: Its effect on the microsomal membrane and sorite membrane bound microsomal enzymes. Eur. J. Biochem. 37: pp. 51-59, 1973.

Hollenberg PF. Mechanisms of cytochrome P-450 and peroxidasc-catalyzed xenobiotic metabolism. The FASEB J. 6: 686-694, 1992.

Hood RD, Naughton MJ and Hayes AW. Prenatal effects of ochratoxin $A$ in hamisters. Teratology 13: 11-14, 1976.

Horton AA and Fairhurst S. Lipid peroxidation and mechanisms of toxicity. CRC Critical Reviews in Toxicology 18: 27-79, 1987.

Hult K, Hokby E, Gatenbeck $S$ and Rutquist L. Ochratoxin A in blood from sliughter pigs in Sweden: Use in evaluation of toxic content of consumed feed. Appl, Environ. Microbiol. 39: 828-830, 1980.

Hult K, Plestina R, Ceovic S, Habazin-Novak V and Radic B. Ochratoxin A in human blood: Analytical results and confimmational tests from a study in connection with Balkan endemic nephropathy. In: Proceedings, V International IUPAC Symposium. Mycoloxins and Phycotoxins. September 1-3, 1982, Vienna, Austria, pp. 338-341. Austrian Chem. Soc., Vienna, 1982a

Hult $\mathrm{K}$, Plestina R, Habazin-Novak V, Radic $B$ and Ceovic S. Ochratoxin A in human blood and Balkan endemic nephropathy. Arch. Toxicol. 51: 313-321, 1982b.

Hult K, Teiling A and Gatenbeck S. Degradation of ochratoxin A by a ruminant. Appl. 
Environ. Microbiol. 32: 443-444, 1976.

Hunter FE, Jr., Gebicki JM, Hoffsten PI, Weinstein J and Scott A. Swelling and lysis of rat liver mitochondria induced by ferrous ions. J. Biol. Chem. 238: pp. 828-835, 1963.

Hutchison RD, Steyn PS and Thompson DL. The isolation and structure of 4-hydroxy ochratoxin A and 7-carboxy-3,4-dihydro-8-hydroxy-3-methyl isocoumarin from Penicillium veridicatum. Tetrahedron Letters 43: 4033-4036, 1971.

Jaeschke $\mathrm{H}, \mathrm{Kleinwaechter} \mathrm{C}$ and Wendel $\mathrm{A}$. The role of acrolein in allyl alcohol-induced lipid peroxidation and liver cell damage in mice. Biochem. Pharmacol. 36: 51-57, 1987.

Jelinek CF, Pohland AE and Wood GE. Worldwide occurrence of micotoxins in foods and feeds, an update. J. Assoc. Off. Anal. Chem. 72: 223-230, 1989.

Jonen HG, Huthwohl B, Kahl R and Kahl GF. Influence of pyridine and some pyridine derivatives on spectral properties of reduced microsomes and on microsomal drug metabolizing activity. Biochem. Pharmacol. 23:1319-1329, 1974.

Jones DP, Thor H, Smith MT, Jewell SA and Orrenius S. Inhibition of ATP-dependent microsomal $\mathrm{Ca}^{2+}$ sequestration during oxidative stress and its prevention by glutathione. $J$. Biol. Chem. 258: 6390-6393, 1983.

Jordan RA and Schenkman JB. Relationship between malondialdehyde production and arachidonate consumption during NADPH-supported microsomal lipid peroxidation Biochem. Pharmacol. 31: 1393-1400, 1982.

Josefsson E. Study of ochratoxin A in pig kidneys. Var. Fda. 31: 415-420, 1979.

Jurima-Romet $\mathrm{M}$ and Huang $\mathrm{HS}$. Enalapril hepatotoxicity in the rat. Effects of modulators of cytochrome P-450 and glutathione. Biochem. Pharmacol. 44(9): 1803-1810, 1992.

Kane A, Creppy EE, Roschenthaler R and Dirheimer G, Changes in urinary and renal tubular enzymes caused by sub-chronic administration of ochratoxin A to rats. Toxicology 
42: $233-243,1986$.

Kanisawa $M$ and Suzuki $S$. Induction of renal and hepatic tumours in mice by ochratoxin A, a mycotoxin. Gann 69: 599-600, 1978.

Kappus $\mathrm{H}$ and Muliawan $\mathrm{H}$. Alkane formation during liver microsomal lipid peroxidation. Biochem. Pharmacol. 31: 597-600, 1982.

Khan S, Martin M and Rahimtula AD. Perturbation of liver microsomal calcium homeostasis by ochratoxin A. Blochem. Pharmacol. 38: 67-72, 1989.

Kitada M, Chiba K, Kamataki T and Kitagawa H. Inhibition by cyanide of drug oxidations in rat liver microsomes. Jpn. J. Pharmacol. 27: 601-608, 1977.

Klinkert W, Lorkowski G, Creppy EE, Dirheimer G and Roschenthaler R. Inhibition of macrophage migration by ochratoxin $\mathrm{A}$ and citrinin, and prevention by phenylalanine of the ochratoxin A-induced inhibition. Toxicol. Eur. Res. 3: 186-189, 1981.

Koppenol WH. The physiological role of the charge distribution on superoxide dismutase. In: Oxygen and Oxy-Radicals in Chemistry and Biology (Rodgers M A J and Powers E L, Eds.), pp. 671-673, Academic Press, New York, 1981.

Koppenol WH. The reaction of ferrous-EDTA with hydrogen peroxide: Evidence against hydroxyl radical formation. Free Rad. Biol. Med. 1: 281-285, 1985.

Krogh P. Ochratoxins: occurrence, biological effects and causal role in disease. In: Natural toxins (Eaker D and Wadstrom T, Eds.), pp. 673.680, Oxford, Pergamon Press, 1980.

Krogh P, Mycotoxic porcine nephropathy - a possible model for Balkan (endemic) nephropathy. In: Proceedings of the second International Symposium on Endemic Nephropathy, Sofia (Pulchev A, Ed.), pp. 266-270, Bulgarian Acadcmy of Sciences, 1974. 
Krogh P, Ochratoxins. In: Mycotoxins in Human and Animal Health (Eds. Rodricks JV, Hesseltine CW and Mehlman MA), pp. 489-498. Pathotox Publishers, New York, 1978.

Krogh P and Neshcim S, Ochratoxin A, Introduction. In: Environmental Carcinogens. Selected Methods of Analysis, Vol. 5, Some Mycotoxins (Stoloff L, Castegnaro M, Scott P, O'Neill IK, and Bartsch H, Eds.), pp. 247-259. IARC Scientific Publications, No. 44, Lyon, 1982.

Kuiper-Goodman T and Scott PM. Risk assessment of the mycotoxin ochratoxin A. Biomed. Environ. Sci. 2: 179-248, 1989.

Kumagai $S$ and Aibara K. Intestinal absorption and secretion of ochratoxin $A$ in the rat. Toxicol. Appl. Pharmacol. 64: 94-102, 1982.

Kunert $\mathrm{KJ}$ and Tappel AL. The effect of vitamin $\mathrm{C}$ on in vivo lipid peroxidation in Guinea pigs as measured by pentane and ethane production. Lipids 18: 271-274, 1983.

Kuo C-H, Maita K, Sleight SD and Hook JB. Lipid peroxidation: A possible mechanism of cephaloridine-induced nephrotoxicity. Toxicol. Appl. Pharmacol. 67: 78-88, 1983.

Kurata H. Current scope of mycotoxin research from the viewpoint of food mycology. In: Toxicology, biochemistry and pathology of mycotoxins (Uraguchi $\mathrm{K}$ and Yamazaki $\mathrm{M}$, Eds.), pp. 162, Kodansha Ltd., Tokyo, 1978.

Laethem RM, Laethem $\mathrm{CL}$ and Koop DR. Purification and properties of a cytochrome P450 arachidonic acid epoxygenase from rabbit renal cortex. J. Biol. Chem. 267(8):5552$5559,1992$.

Lake BG. Preparation and characterization of microsomal fractions for studies on xenobiotic metabolism. In: Biochemical Toxicology (Eds. Snell K and Mulloch B), pp. 183-215. IRL Press Lid., Oxford, England, 1987.

Lawrence GD and Cohen G. Ethane exhalation as an index of in vivo lipid peroxidation: Concentrating cthane from a breath collection chamber. Anal. Biochem. 122: 283-290, 
1982.

Lea T, Steien K and Stormer FC. Mechanism of ochratoxin A-induced immunosuppression. Mycopathologia 107: 153-159, 1989.

Lee SC, Berry JT and Chu FS. Immunohistochemical fate of ochratoxin $A$ in mice. Toxicol. Appl. Pharmacol. 72; 218-227, 1984.

Levi CP, Trenk HL and Mohr HK. Study of the occurrence of ochratoxin A in green coffec beans. J. Assoc. Off. Anal. Chem. 57: 866-870, 1974.

Lowry $\mathrm{OH}$, Rosebrough NJ, Farr AL and Randell RJ. Protein measurement with the Folin phenol reagent. J. Biol. Chem. 193: 265-275, 1951.

Lubet AR, Mayer RT, Cameron JW, Nims RW, Burke MD, Wolff T and Guengerich FP. Dealkylation of pentoxyresorufin: $A$ rapid and sensitive assay for measuring induction of cytochrome(s) P-450 by phenobarbital and other xenobiotics in the rat. Arch. Biochem. Biophys. 238(1): 43-48, 1985.

Luster MI, Germolec DR, Burleson GR, Jameson CW, Ackermann MF, Lamrn KR and Mayer HT. Selective immunosuppression in mice of natural killer cell activity by ochratoxin A. Cancer Res. 47: 2259-2263, 1987.

Ma X-L, Baraona E, Lasker JM and Lieber CS. Effects of ethanol consumption on bioactivation and hepatotoxicity of $\mathrm{N}$-nitrosodimethylamine in rats. Biochem. Pharmacol. 42: 585-591, 1991.

Marquardt $\mathrm{R}$ and Frohlich $\mathrm{A}$. A review of recent advances in understanding ochratoxicosis. J. Anim. Sci. 17: 3968-3988, 1992.

Marquardt RR, Frolich AA, Sreemannarayana O, Abramson D and Bernatsky A. Ochratoxin A in blood from slaughter pigs in western Canada. Can. J. Vet. Res. 52: 186$190,1988$. 
Marquardt RR, Frohlich A and Abramson D. Ochratoxin A: an important western Canadian storage mycotoxin. Can. J. Physiol. Pharmacol. 68: 991-999, 1990.

May HE and McCay PB. Reduced diphosphopyridine nucleotide oxidase-catalyzed alterations of membrane phospholipids. J. Biol. Chem. 243: 2296-2305, 1968.

Mayura K, Reddy RV, Hayes AW and Berndt WO. Embryocidal, fetotoxic and teratogenic effects of ochratoxin A in rats. Toxicol. 25: 175-185, 1982.

Meisner H. Energy-dependent uptake of ochratoxin A by mitochondria. Arch. Biochem. Biophys. 173: 132-140, 1976.

Meisner $\mathrm{H}$ and Chan S. Ochratoxin A, an inhibitor of mitochondrial transport system. Biochem. 13: 2795-2800, 1974.

Meisner H, Cimbala MA and Hanson RW. Decrease of phosphoenolpyruvate carboxylase RNA and poly(A)RNA level by ochratoxin A. Arch. Biochem. Biophys. 223: 264-270, 1983.

Meisner $\mathrm{H}$ and Krogh P. Phosphoenolpyruvate carboxykinase as a selective indicator of ochratoxin A induced nephropathy. Dev. Toxicol. Environ. Sci. 14: 199-206, 1986.

Meisner $\mathrm{H}$ and Meisner $\mathrm{P}$, Ochratoxin A, an in vivo inhibitor of renal phosphoenolpyruvate carboxykinase. Arch. Biochem. Biophys. 208: 146-153, 1981.

Meisner $\mathrm{H}$ and Polsinelli $\mathrm{L}$. Changes of renal mRNA species abundance by ochratoxin $\mathrm{A}$. Biochem. Pharmacol. 35: 661-665, 1986.

Meisner $\mathrm{H}$ and Selanik $\mathrm{P}$. Inhibition of renal gluconeogenesis in rats by ochratoxin. Biochem. J. 180: 681-684, 1979.

Ministry of Agriculture, Fisheries and Food. Survey of mycotoxins in the United Kingdom, London, Ministry of Agriculture, Fisheries and Food, Food Surveillance Paper No. 4. 
Minotti $\mathbf{G}$ and Aust SD. The role of iron in the initiation of lipid peroxidation. Chem. Phys. Lipids 44: 191-208, 1987a.

Minotti G and Aust SD. The requirement of Iron (III) in the initiation of lipid peroxidation by Iron (II) and hydrogen peroxide. J. Biol. Chem. 262: 1098-1104, 1987 b.

Mishin V, Pokrovsky A and Lyakhovich VV. Interactions of some acceptors with superoxide anion radicals formed by the NADPH-specific flavoprotein in rat liver microsomal fractions. Biochem. J. 154: 307-310, 1976.

Monostory K, Lakatos B and Vereczkey L. Cytochrome P-450: Structure, function, induction and practical significance. Acta Pharm. Hung. 62(6): 318-332, 1992.

Moore JH and Truelove. Ochratoxin A, inhibition of mitochondrial respiration. Science (Washington DC) 168: 1102, 1970.

Morehouse LA, Tien M, Bucher JR and Aust SD. Effect of hydrogen peroxide on the initiation of microsomal lipid peroxidation. Biochem. Pharmacol. 32: 123-127, 1983.

Morehouse LA and Aust SD. Reconstitutad microsomal lipid peroxidation: ADP-Fe ${ }^{3+}$ dependent peroxidation of phospholipid vesicles containing NADPH-cytochrome P-450 reductase and cytochrome P-450. Free. Rad. Biol. Med. 4: 269-277, 1988.

Moroi K, Suzuki S, Kuga T, Yamazaki M and Kanisawa M. Reduction of ochratoxin A toxicity in mice treated with phenylalanine and phenobarbital. Toxicol. Lett. 25:1-5, 1985.

Muller A and Sies H. Assay of ethane and pentane from isolated organs and cells. In: Methods in Enzymology (Packer L, Ed.), Vol. 105: pp. 311-319, Academic Press, New York, 1984.

Murray Ardies, Lasker JM, Bloswick BP and Lieber CS. Purification of NADPI1cytochrome $\mathrm{C}$ reductase from hamster liver microsomes by detergent extraction and affinity 
chromatography. Anal. Biochem. 162: 39-46, 1987.

Nakajima T, Elovaara E, Park SS, Gelboin HH, Hietanen E and Vainio H. Monoclonal antibody-directed characterization of benzene, ethoxyresorufin and pentoxyresorufin metabolism in rat liver microsomes. Biochem. Pharmacol. 40: 1255-1261, 1990.

Nakajima T, Wang R-S, Elovaara E, Park SS, Gelboin HV, Hietanen E and Vainio H. Monoclonal antibody-directed characterization of cytochrome $\mathrm{P}-450$ isozymes responsible for toluene metabolism in rat liver. Biochem. Pharmacol. 41: 395-404, 1991.

Narabayashi H, Takeshige $\mathrm{K}$ and Minakami S. Alterations of inner-membrane components and damage to electron-transfer activities of bovine heart submitochondrial particles by NADPH-dependent lipid peroxidation. Biochem. J. 202: 97-105, 1982.

Nebert DW, Adesnik M, Coon MJ, Estabrook RW, Gonzalez FJ, Guengerich FP, Gunsalus IC, Johnson EF, Kemper B, Levin W, Phillips IR, Sato R and Waterman MR. The cytochrome P.450 gene superfamily: Recommended nomenclature. DNA and Cell Biol. 6: 1-11, 1987.

Ngaha EO and Plummer DT. Toxic renal damage: Changes in enzyme levels. Biochem. Med. 18: pp. 71, 1977.

Niehaus WG and Samuelsson B. Formation of malondialdehyde from phospholipid arachidonate during microsomal lipid peroxidation. Eur. J. Biochem. 6: 126-130, 1968.

O'Brien PJ. Intracellular mechanisms for the decomposition of a lipid peroxide. I. Decomposition of a lipid peroxide by metal ions, heme compounds, and nucleophiles. Can. J. Biochem. 47: 485-492, 1969.

O'Brien PJ. Radical formation during the peroxidase catalyzed metabolism of carcinogens and xenobiotics: the reactivity of these radicals with GSH, DNA, and unsaturated lipid. Free. Rad. Biol. Med. 4: 169-183, 1988.

O'Connell MJ and Garner A. Radiation-induced generation and properties of lipid 
hydroperoxide in liposomes. Int. J. Radiation Biol. 44: 615-625, 1983.

Omar RF. Mechanism of ochratoxin A stimulated lipid peroxidation. A Master's thesis. Biochem. Dept., Memorial University, St. John's, NF, Canada, 1990.

Omar RF, Hasinoff BB, Mejilla F and Rahimtula AD. Mechanism of ochratoxin A stimulated lipid peroxidation. Biochem. Pharmacol. 40: 1183-1191, 1990.

Omura $\mathrm{T}$ and Sato $\mathrm{R}$. The carbon mcnoxide-binding spectrum of liver microsomes. $J$. Biol. Chem. 239: 2379-2384, 1964.

Orrenius $\mathrm{S}$ and Bellomo G. Toxicological implications of perturbation of $\mathrm{Ca}^{2+}$ homeo:tasis in hepatocytes. In: Calcium and cell function (Cheung WY, Ed.), pp. 185 Academic Press, Orlando, Fl., 1986.

Ortiz de Montellano PR and Reich NO. Inhibition of cytochrome P-450 enzymes. In: Cytochrome P-450: Structure, mechanism and biochemistry (Ortiz de Montellano, Ed.) Ch.8, pp. 273-314, Plenum Press, New York and London, 1986.

Ortiz de Montellano PR and Correia MA. Suicidal destruction of cytochrome P-450 during oxidative drug metabolism. Annu. Rev. Pharmacol. Toxicol. 23: 481-503, 1983.

Osborne BG. The occurrence of ochratoxin A in mouldy bread and flour. Food Cosmet. Toxicol. 18: 615-617, 1980.

Oster T, Jayyosi Z, Creppy EE, S. El Amri H and Batt A-M. Characterization of pig liver purified cytochrome P-450 isozymes for ochratoxin A metabolism studies. Toxicol. Lett. 57: 203-214, 1991.

Palmer DN, Rabin BR and Williams DJ. A subpopulation of rat liver membrane-bound ribosomes that are detached in vitro by carcinogens and centrifugation. Biochem. I. 176: 9-14, 1978.

Parkinson A, Thomas PE, Ryan DE, Gorsky LD, Shively JE, Sayer JM, Jerina DM and 
Levin W. Mechanism of inactivation of rat liver microsomal cytochrome P-450c by 2Bromo-4'-nitroacetophenone. J. Biol. Chem. 261(25): 11487-11495, 1986.

Peckham JC, Doupnik Jr. B and Jones Jr. OH, Acute toxicity of ochratoxins A and B in chicks. Appl. Microbiol. 21: 492-494, 1971.

Pederson TC, Buege JA and Aust SD. Microsomal electron transport. The role of NADPH-cytochrome $C$ reductase in liver microsomal lipid peroxidation. J. Biol. Chem. 248:7134-7141, 1973.

Pederson TC and Aust SD. NADPH-dependent lipid peroxidation catalyzed by purified NADPH-cytochrome c reductase from rat liver microsomes. Biochem. Biophys. Res. Commun. 48: 789-795, 1972.

Petkova-Bocharova T, Chernozemsky IN and Castegnaro $\mathrm{M}$. Ochratoxin A in human blood in relation to Balkan endemic nephropathy and urinary system tumors in Bulgaria. Food Additives and Contamination 5: 299-301, 1988.

Poli G, Albano E and Dianzani MV. The role of lipid peroxidation in liver damage. Chem. Phys. Lipids 45: 117-142, 1987.

Price RG. Urinary enzymes, nephrotoxicity, and renal disease. Toxicology 23: 99-134. 1982.

Prior MG. Mycotoxin in determinations on animal feedstuffs and tissues in Western Canada. Can J. Comp. Med. 40: 75-79, 1976.

Pryor WA and Castle L. Chemical methods for the detection of lipid hydroperoxides. In: Methods in Enzymology (Packer L, Ed.), Vol 105: pp. 293-299 , Academic Press, New York, 1984.

Purchnse IFH and Theron JJ, The acute toxicity of ochratoxin A to rats. Food Cosmet. Toxicol. 6: 479-483, 1968. 
Rahimtula AD, Bereziat J-C, Busscchini-Griot V and Bartsch H. Lipid peroxidation as a possible cause of ochratoxin A toxicity. Biochem. Pharmacol. 37: 4469-4477, 1988.

Rahimtula AD, Zachariah PK and O'Brien PJ. Differencial effects of antioxidants, steroids and other compounds on benzo(a)pyrene 3 hydroxylase activities in various tissues of rat. Br. J. Cancer 40:105-112, 1979.

Rahimtula AD and O'Brien PJ. Hydroperoxide catalyzed liver microsomal aromitic hydroxylation reactions involving cytochrome P-450. Biochem. Biophys. Res. Commun. 60: $440-447,1974$.

Rahimtula AD and O'Brien PJ. Hydroperoxide dependent O-dealkylation reactions catalyzed by liver microsomal cytochrome P-450. Biochem. Biophys. Res. Commutn. 62: 268-275, 1975.

Recknagel RO, Glende EA, Waller RL and Lowrey K. Lipid peroxidation: Biochemistry, measurement, and significance in liver cell injury. In: Toxicology of the Liver (Plia G and Hewitt IV R, Eds.), pp. 213-241, Raven Press, New York, 1982.

Recknagel RO and Glende EA, Jr. Spectrophotometric detection of lipid conjugated dienes. Methods in Enzymology, Vol. 105: 331-337 (Packer L, Ed.), Academic Press, New York, 1984.

Rex-DK and Kumar S. Recognizing acetaminophen hepatotoxicity in chronic alcoholics. Postgrad. Med. 91: 241-245, 1992.

Roschenthaler R, Creppy EE and Dirheimer G. Ochratoxin A: On the mode of action of an ubiquitous mycotoxin. J. Toxicol. 3: 53-86, 1984.

Ryan DE, Ramanathan L, Iida S, Thomas PE, Haniu M, Shively JE, Lieber CS and Levin W. Characterization of a major form of rat hepatic microsomal cytochrome P-450 induced by isoniazid. J. Biol. Chem. 260: 6385-6393, 1985.

Sandy MS, Moldeus P, Ross D and Smith M. Role of redox cycling and lipid peroxidation 
in bipyridyl herbicide cytotoxicity. Studies with a compromised isc lated hepatocyte model system. Biochem. Pharmacol. 35: 3095-3101, 1986.

Scott PM, Van Walbeek W, Kennedy B and Anyeti D. Mycotoxins (ochratoxin A, citrinin and stregmatocystin) and toxigenic fungi in grains and other agricultural products. $J$. Agric. Food Chem. 20: 1103-1109, 1972.

Shotwell OL, Goulden ML, Hesseltine CW. Survey of US wheat for ochratoxin and aflatoxin. J. Assoc. Off. Anal. Chem. 59: 122-124, 1976.

Shreeve BJ, Patterson DSP and Roberts BA. The carry-over of aflatoxin, ochratoxin and zearalenone from naturally contaminated feed to tissues, urine and milk of dairy cows.

Food Cosmet. Toxicol. 17: 151-152, 1979.

Sies H (Ed.). Oxidative Stress, Academic Press, London, 1985.

Silverblatt F, Turck $M$ and Bulger $R$. Nephrotoxicity due to cephaloridine: A light- and electron-microscopic study in rabbits. J. Infect. Dis. 122:33-44, 1970.

Slater TF (Ed.). Free Radical Mechanisms in Tissue Injury, Pion Press Ltd., London, 1972.

Slater TF. Activation of carbon tetrachloride: Chemical principles and biological significance. In: Free Radicals, Lipid Peroxidation and Cancer (McBrien D CH and Slater TF, Eds.), pp. 243-274, Academic Press, London, 1982.

Smith JE and Moss MO (Eds.). Structure and formation of mycotoxins. In: Mycotoxins: formation, analysis and significance, pp. 31-49, 1985.

Su kol P, Ripich G, Holohan P and Ross C. Mechanism of ochratoxin A transport in kidney. J. Pharmacol. Exp. Ther. 246: 460-465, 1988.

Soucek P and Gut I. Cytochromes P-450 in rats: structures, functions, properties and relevant human forms. Xenobiotica 22: 83-103, 1992. 
Spaethe SM and Jollow DJ. Effect of cobalt protoporphyrin on hepatic drug metabolizing enzymes. Biochem. Pharmacol. 38: 2027-2038, 1989.

Steyn PS and Holzapfel CW. The isolation of the methyl and ethyl esters of ochratoxin A and B metabolites of Aspergillus ochraceus Wilh. J. S. Afr. Chem. Inst. 20: 186-189, 1967.

Stormer FC, Storen O, Hansen CE, Pedersen JI, and Aasen AJ. Formation of $4(\mathrm{R})$ - and 4(S)-4-OH-ochratoxin A and 10-OH-ochratoxin A from ochratoxin A by rabbil liver microsomes. Appl. Environ. Microbiol. 45: 1183-1187, 1983.

Stormer FC, Hansen CE, Pedersen JI, Hvistendahl G and Aasen AJ. Formation of 4(R)and 4(S)-4-OH-ochratoxin A from ochratoxin A by liver microsomes from various species. Appl. Environ. Microbiol. 42: 1051- 1056, 1981.

Stormer FC and Pedersen JI. Formation of 4-OH-ochratoxin A from ochratoxin $\wedge$ by rat liver microsomes. Appl. Environ. Microbiol. 39: 971-975, 1980.

Subramanian S, Kanthrsamy A, Balasubramanian N, Sekar N and Govindasamy S. Ochratoxin A toxicity on carbohydrate metabolism in rats. Bull. Environ. Contum. Toxicol. 43: 180-184, 1989.

Suzuki S, Satoh T and Yamazaki M. Effect of ochratoxin A on carbohydrate metabolism in rat liver. Toxicol. Appl. Pharmacol. 32: 116-122, 1975.

Svingen BA, O'Neal FO and Aust SD. The role of superoxide and singlet oxygen in lipid peroxidation. Photochem. Photobiol. 28: pp. 803, 1978.

Szczech GM, Carlton WW and Caldwell R. Ochratoxin A toxicosis in swine. Vet. Pathol. 10: $347-364,1973$.

Testa B and Jenner P. Inhibitors of cytochrome P-450s and their mechanism of action. Drug Met. Rev. 12: 1-117, 1981. 
Tien M, Svingen BA and Aust SD. Initiation of lipid peroxidation by perferryl complexes. In: Oxygen and Oxy-radicals in Chemistry and Biology (Rodgers MAJ and Powell EL, Eds.), pp. 147-152. Academic Press, New York, 1981.

Tomasi A, Billing S, Garner A, Slater TF and Albano E. The metabolism of halothane by hepatocytes: A comparison between free radical spin trapping and lipid peroxidation in relation to cell damage. Chem. Biol. Interact. 46: 353-368, 1983.

Tsubouchi H, Yamamoto K, Hisada K, Sakabe $\mathrm{Y}$ and Udagawa S. Effect of roasting on ochratoxin $A$ level in grcen coffee beans inoculated with Aspergillus ochraceus. Mycopatkologia 97: 111-115, 1987.

Tune BM and Fravert D. Mechanisms of cephaloridine nephrotoxicity, A comparison of cephaloridine and cephaloglycin. Kidney Int. 18: 591-600, 1980.

Ueno Y. The toxicology of mycotoxins. CRC Crit. Rev. Toxicol. 14: 99-132, 1985a.

Ueno Y. Biotransformation of mycotoxins in the reconstituted cytochrome P-450 system. Proc. Jpn. Assoc. Mycotoxicol. 22: 28-30, 1985.

Ursini F, Maiorino M, Hochstein P and Emster L. Microsomal lipid peroxidation: Mechanisms of initiation. The role of iron chelators. Free Rad. Biol. Med. 6: 31-36, 1989.

Van der Merwe KJ, Steyn PS and Fourie L. The constitution of ochratoxin A, B and C metabolites of Aspergillus ochraceus Wilh. J. Chem. Soc. 7083-7088, 1965 b.

Van der Merwe KJ, Steyn PS, Fourie L, De Scott B and Theron JJ. Ochratoxin A, a toxic metabolite produced by Aspergillus ochraceus Wilh. Nature (Lond) 205: 112-113, 1965a.

Vesela D, Vesely D, Jelinck R, and Kusak, V. Detection of ochratoxia A in feed barley. Vet. Mcd. 23: 431-436, 1978.

Vladimirov YA, Olenev VI, Suslova TB and Cheremisina ZP. Lipid peroxidation in 
mitochondrial membrane. In: Advances in Lipid Research (Paoletti R and Kritchevsky D, Eds.), Vol. 7, pp. 173-249, Academic Press, New York, 1980.

Wehner FC, Thiel PG, van Rensburg SJ and Demasius IPC, Mutagenicity to Salmonella typhimurium of some Aspergillus and Penicillium mycotoxins. Mutat. Res. 58: 193-203, 1978.

Wei YH, Lu C-Y, Lin T-N and Wei RD. Effect of ochratoxin A on rat liver mitochondrial respiration and oxidative phosphorylation. Toxicol. 36: 119-130, 1985.

Wendel A and Feuerstein S. Drug induced lipid peroxidation in mice -I: Modulation of monooxygenase activity, glutathione and selenium status. Biochem. Pharmacol. 30: 2513 2520,1981 .

Wemer M, Maruhn D and Atoba $\mathrm{M}$. Use of gel filtration in the assay of urinary enzymes. J. Chronatography 40: 254-263, 1969.

Wiebel FJ, Leutz JC, Diamond L and Gelboin HV. Aryl hydrocarbon (Benzo[alpyrene) hydroxylase in microsomes from rat tissues: Differential inhibition and stimulation by benzoflavone and organic solvents. Arch. Biochem. Biophys. 144: 78-86, 1971.

Wills ED and Wilkinson AE. Release of enzymes from lysosomes by imadiation and the relation of lipid peroxide formation to enzymic release. Biochem. J. 99: 657-666, 1966.

Wislocki PG, Miwa GT and Lu AYH. Reactions catalyzed by the cytochrome P-450 system. In: Enzymatic basis of detoxification (Jakoby WB, Ed.) Vol. 1, pp. 135-182. Academic Press, New York, 1980.

Woolf $\mathrm{L}$. The heterozygot advantage in phenylketonuria. Am. J. Human Genctics 38: 773-775, 1986.

World Health Organization. Environmental Health Criteria 105: Selected mycotoxins: Ochratoxins, trichothecenes, ergot. WHO, Geneva, 1990. 
Yamazaki $\mathrm{H}$, Inui $\mathrm{Y}$, Yun $\mathrm{CH}$, Guengerich FP, Shimada T. Cytochrome P-450 2E1 and $2 A 6$ enzymes as major catalysts for metabolic activationof $\mathrm{N}$-nitrosodialkylamines and tobacco-related nitrosamines in human liver microsomes. Carcinogenesis 13(10): 1789 $1794,1992$. 


